University of Tennessee Health Science Center

UTHSC Digital Commons

\title{
$5-2014$
}

\section{Development of a Novel Vaccine Adjuvant System Utilizing an In Situ Implant System to Modified Release}

Qiuye Zhang

University of Tennessee Health Science Center

Follow this and additional works at: https://dc.uthsc.edu/dissertations

Part of the Pharmaceutics and Drug Design Commons

\section{Recommended Citation}

Zhang, Qiuye , "Development of a Novel Vaccine Adjuvant System Utilizing an In Situ Implant System to Modified Release" (2014). Theses and Dissertations (ETD). Paper 321. http://dx.doi.org/10.21007/ etd.cghs.2014.0376.

This Dissertation is brought to you for free and open access by the College of Graduate Health Sciences at UTHSC Digital Commons. It has been accepted for inclusion in Theses and Dissertations (ETD) by an authorized administrator of UTHSC Digital Commons. For more information, please contact jwelch30@uthsc.edu. 


\title{
Development of a Novel Vaccine Adjuvant System Utilizing an In Situ Implant System to Modified Release
}

\begin{abstract}
Pulsatile release formulations for single dose vaccines have been studied for many years because of the advantages that they may provide to vaccine administration with a single dose instead of prime and booster shots.

The aim of this work is to develop novel formulations based on an In Situ Implant (ISI) systems to provide a modified vaccine release and altered immune response, which could act as a booster administration. This type of system was selected because ISI systems are used for long acting administration of various drugs and ease of administration. A typical ISI system is comprised of hydrophilic organic solvent (e.g. NMethyl-2-Pyrrolidone, NMP) and hydrophobic polymer (e.g. poly (lactide-co-glycolide), PLGA). We have given this concept the name of AdjuGel to distinguish it from simple drug administration. It was developed by incorporating an oil (e.g. Acetyl Tributyl Citrate, ATBC) into an ISI system. When loaded with dissolved or suspended antigens, AdjuGel can be injected by syringe into the body. After injection, AdjuGel will solidify due to the dissipation of hydrophilic solvent and form an in situ semi-solid implant, which can hold oil and antigen for a delayed release.
\end{abstract}

It was found that an AdjuGel system, composed of ethyl acetate, ATBC and a hydrophobic polymer, such as PLGA or PLA, could stimulate delayed immune response. The delayed period for immune response was adjustable, which was determined by the degradation rates of polymers. A slower polymer degradation rate provided a longer delayed period for immune response.

\section{Document Type}

Dissertation

Degree Name

Doctor of Philosophy (PhD)

\section{Program}

Pharmaceutical Sciences

\section{Research Advisor}

James R. Johnson, PhD.

\section{Keywords}

In Situ Implant, PLGA, Single dose vaccine, Vaccine adjuvant

\section{Subject Categories}

Medicine and Health Sciences | Pharmaceutics and Drug Design | Pharmacy and Pharmaceutical Sciences 


\title{
DEVELOPMENT OF A NOVEL VACCINE ADJUVANT SYSTEM UTILIZING
} AN IN SITU IMPLANT SYSTEM TO MODIFIED RELEASE

\author{
A Dissertation \\ Presented for \\ The Graduate Studies Council \\ The University of Tennessee \\ Health Science Center
}

\author{
In Partial Fulfillment \\ Of the Requirements for the Degree \\ Doctor of Philosophy \\ From The University of Tennessee
}

By

Qiuye Zhang

May 2014 
All material (c) 2014 by Qiuye Zhang. All rights reserved. 


\section{DEDICATION}

I dedicate this work to my wife $\mathrm{Yu}$ Zhao and my children George, Jayson and Annabella for their love and support. 


\section{ACKNOWLEDGEMENTS}

I would like to thank my former research advisor and mentor, Dr. Atul Shukla, who introduced and guided me into this amazing project. Dr. Shukla passed away in 2009 and we all missed him greatly.

I would also like to express my deepest gratitude to my research advisor Dr. James Johnson, for his supervision, advice and encouragement for the research projects. Without his support, I cannot finish this difficult project. I also thank him for the time he spent guiding me writing, editing and revising the dissertation.

I would also thank the members in my committee, Drs. Timothy Mandrell, Hassan Almoazen, Richard Webby, Elizabeth Fitzpatrick, and Fabio Re for their valuable and critical comments and advice on my research proposal, qualifying exam and dissertation formulation.

During my Ph.D. studies, I have benefited from all my labmates: Dr. Wen Qu, Mr. Ed Brunson, Dr. Rui Zhu, Dr. Jin Xu, Dr. Yinqi Zhou, Dr. Suresh Potharaju, Mr. Hao Lou, Dr. Wenli Lu and Dr. Sonia Bedi. They helped me a lot with drug formulations.

Special acknowledgement will go to Stacey Barnett, Dr Jackson Scott, Ernestine and Dr. Tim Mandrell for support and guidance for the animal studies. 


\begin{abstract}
Pulsatile release formulations for single dose vaccines have been studied for many years because of the advantages that they may provide to vaccine administration with a single dose instead of prime and booster shots.

The aim of this work is to develop novel formulations based on an In Situ Implant (ISI) systems to provide a modified vaccine release and altered immune response, which could act as a booster administration. This type of system was selected because ISI systems are used for long acting administration of various drugs and ease of administration. A typical ISI system is comprised of hydrophilic organic solvent (e.g. NMethyl-2-Pyrrolidone, NMP) and hydrophobic polymer (e.g. poly (lactide-co-glycolide), PLGA). We have given this concept the name of AdjuGel to distinguish it from simple drug administration. It was developed by incorporating an oil (e.g. Acetyl Tributyl Citrate, ATBC) into an ISI system. When loaded with dissolved or suspended antigens, AdjuGel can be injected by syringe into the body. After injection, AdjuGel will solidify due to the dissipation of hydrophilic solvent and form an in situ semi-solid implant, which can hold oil and antigen for a delayed release.
\end{abstract}

It was found that an AdjuGel system, composed of ethyl acetate, ATBC and a hydrophobic polymer, such as PLGA or PLA, could stimulate delayed immune response. The delayed period for immune response was adjustable, which was determined by the degradation rates of polymers. A slower polymer degradation rate provided a longer delayed period for immune response. 


\section{TABLE OF CONTENTS}

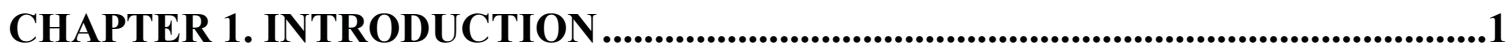

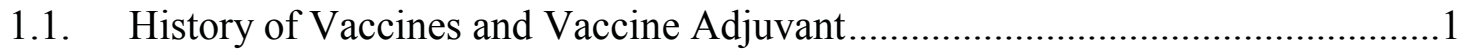

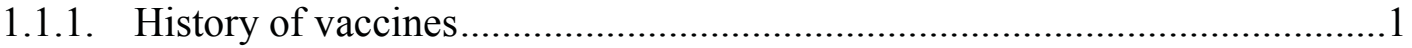

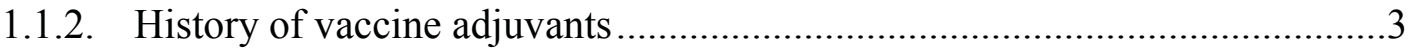

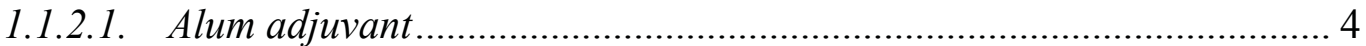

1.1.2.2. Oil based adjuvants ....................................................................... 4

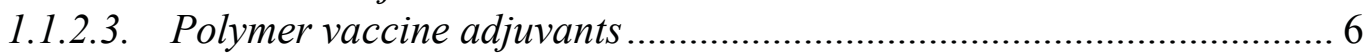

1.1.3. What's the ideal vaccine adjuvant?...........................................................

1.2. Current Status for Development of Adjuvants for Single Dose Vaccine ............8

1.3. What Is the ISI (In Situ Implant) System and Why Was It Chosen for Modifying Antigen Release?.........................................................................

\section{CHAPTER 2. DEVELOPMENT OF NEW VACCINE FORMULATIONS WITH STRONG ADJUVANTICITY BASED ON ISI SYSTEM .............................................16}

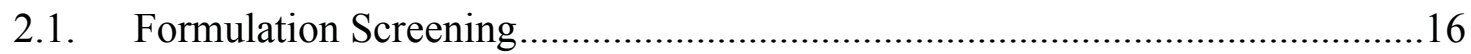

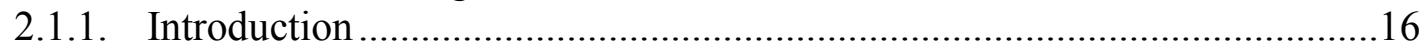

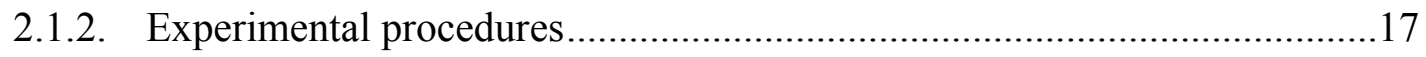

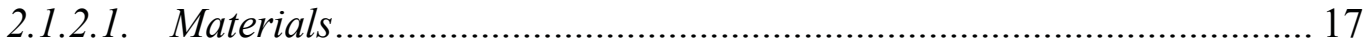

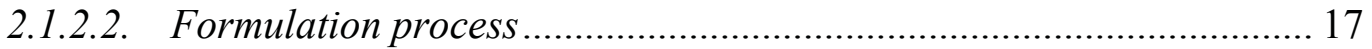

2.1.2.3. Immunization of animals ................................................................ 17

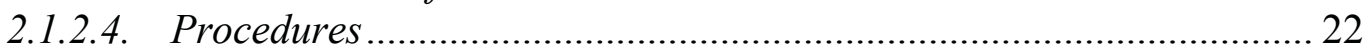

2.1.2.5. Enzyme linked immunosorbent assay (ELISA) ................................. 22

2.1.2.6. Statistical analysis ...................................................................... 22

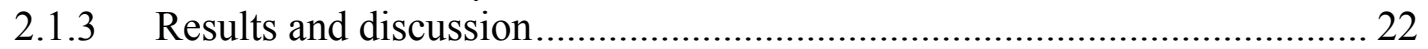

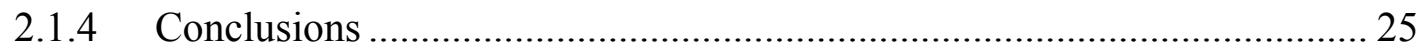

2.2. Study the AdjuGel System and Determine If PLGA Is Necessary for

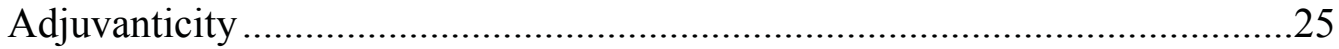

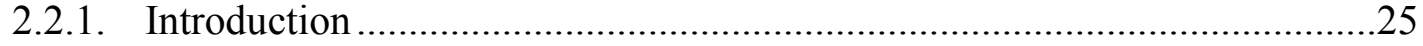

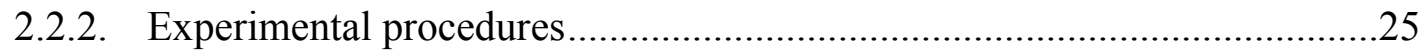

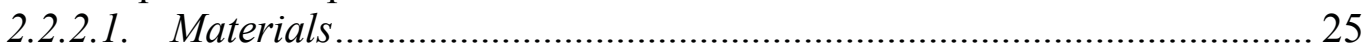

2.2.2.2. Formulation process ......................................................................... 26

2.2.2.3. Immunization of animals ............................................................. 26

2.2.2.4. Procedures .............................................................................. 26

2.2.2.5. Enzyme linked immunosorbent assay (ELISA) ................................... 26

2.2.2.6. Statistical analysis .................................................................... 28

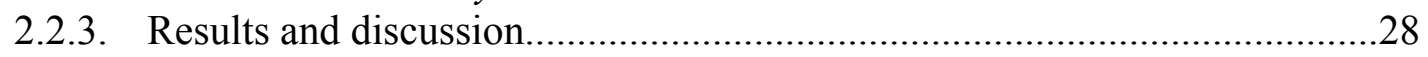

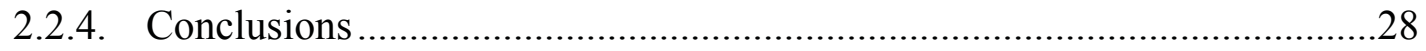

2.3. Study the AdjuGel System and Determine If NMP Is Necessary for

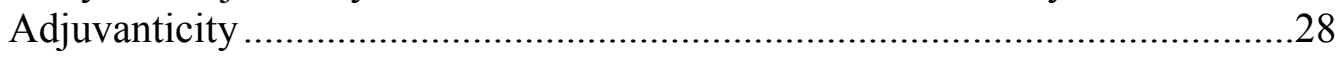

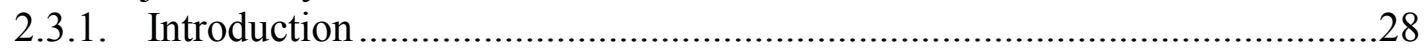

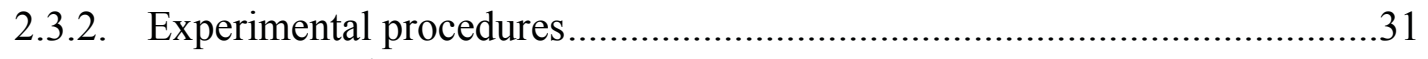

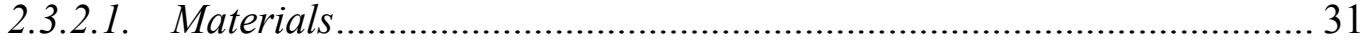

2.3.2.2. Formulation process ……………………………………………..... 31 


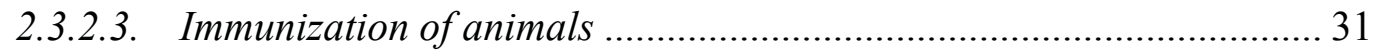

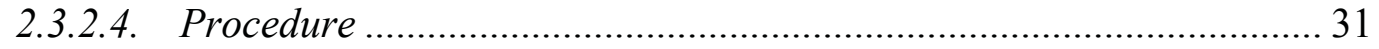

2.3.2.5. Enzyme linked immunosorbent assay (ELISA) ................................... 31

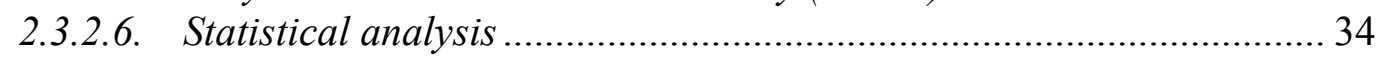

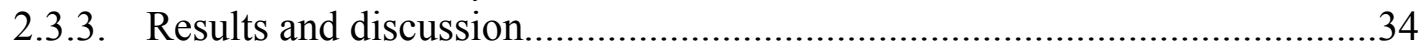

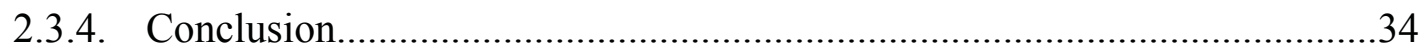

\section{CHAPTER 3. DEVELOPMENT OF NEW VACCINE FORMULATIONS WITH DELAYED IMMUNE RESPONSE BASED ON ADJUGEL SYSTEM USING ORGANIC SOLVENT NMP ..........................................................................................37}

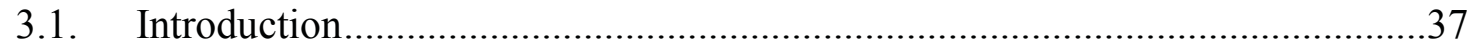

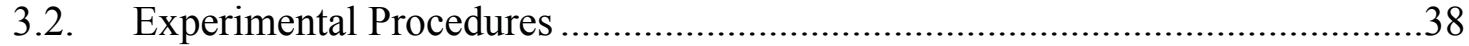

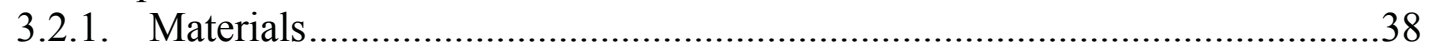

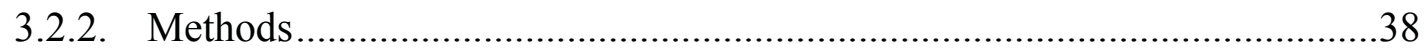

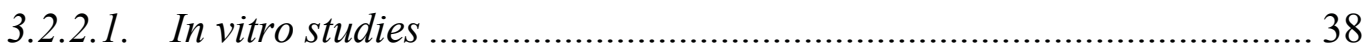

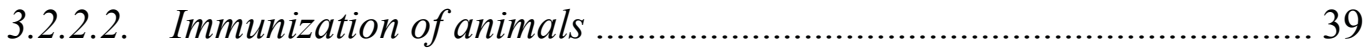

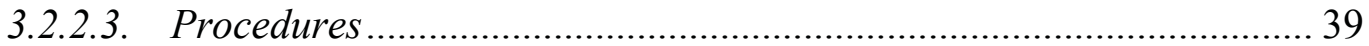

3.2.2.4. Enzyme linked immunosorbent assay (ELISA) ................................... 39

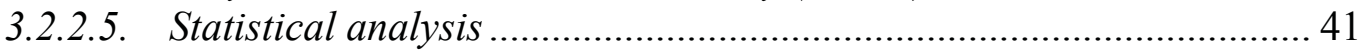

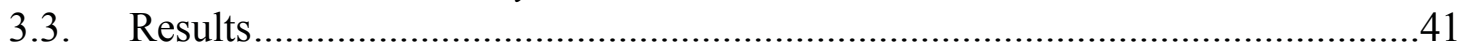

3.3.1. In vitro release- Ovalbumin ................................................................ 41

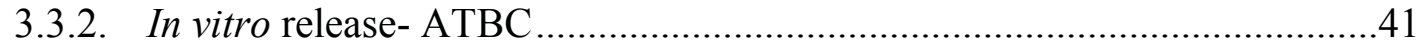

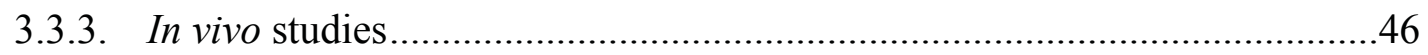

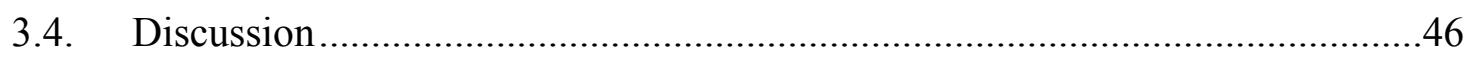

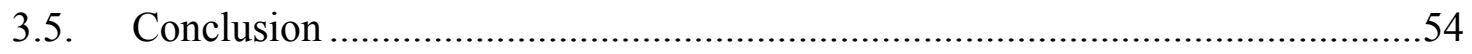

\section{CHAPTER 4. DEVELOPMENT OF NOVEL FORMULATIONS WITH DELAYED IMMUNE RESPONSE BASED ON ADJUGEL SYSTEM USING ORGANIC SOLVENT ETHYL ACETATE...................................................................56}

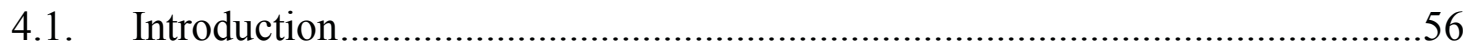

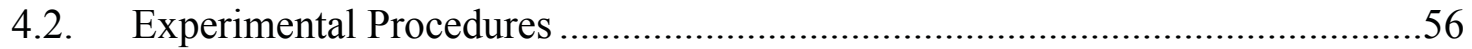

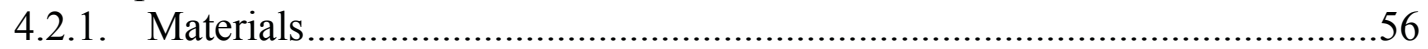

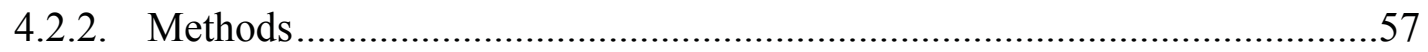

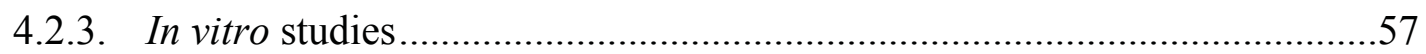

4.2.3.1. Analytical method development for OVA (EIA) .................................. 60

4.2.3.2. Analytical method development for ATBC (HPLC) ............................. 60

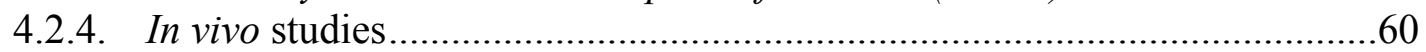

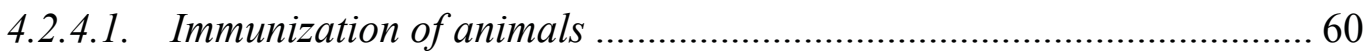

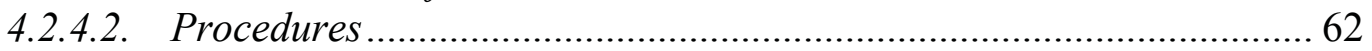

4.2.5. Enzyme linked immunosorbent assay (ELISA) .........................................62

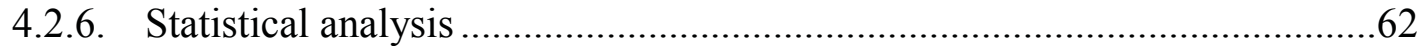

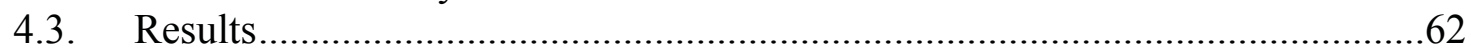

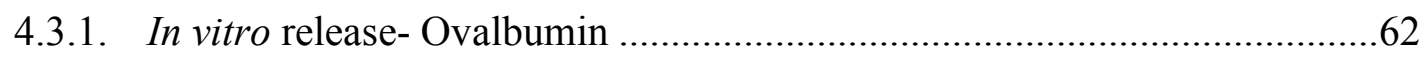

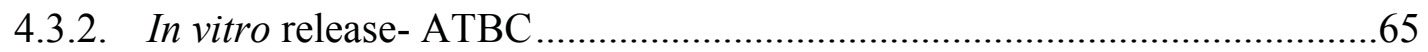

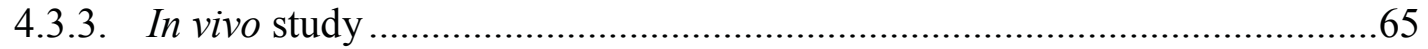

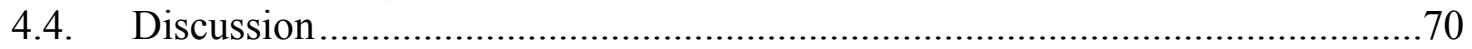


4.5. Conclusion

CHAPTER 5. DEVELOPMENT OF SINGLE DOSE VACCINE WITH THE

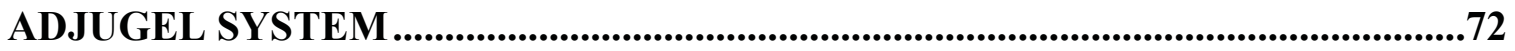

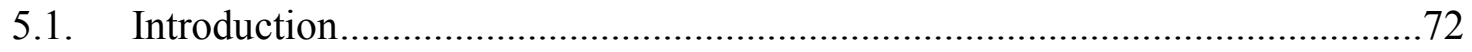

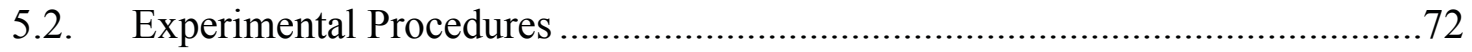

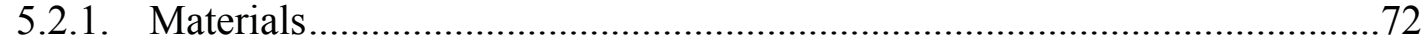

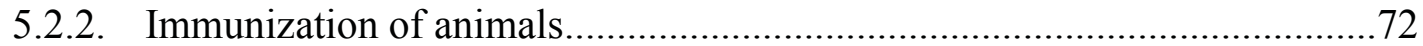

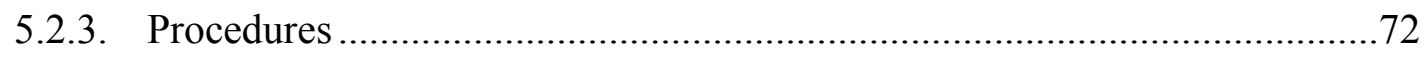

5.2.4. Enzyme linked immunosorbent assay (ELISA) .....................................74

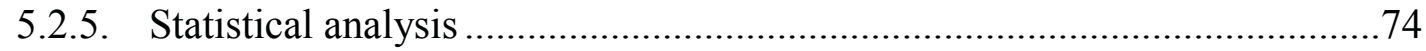

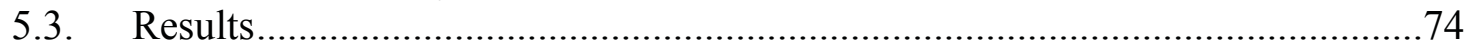

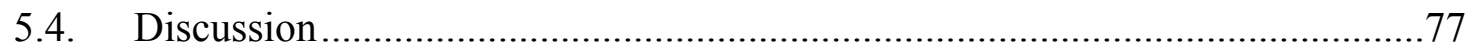

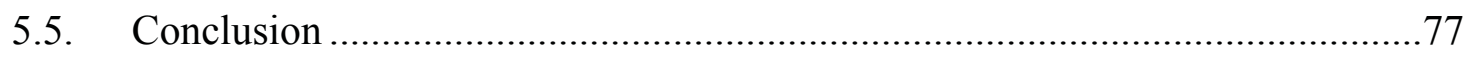

CHAPTER 6. RESEARCH SUMMARY ..........................................................78

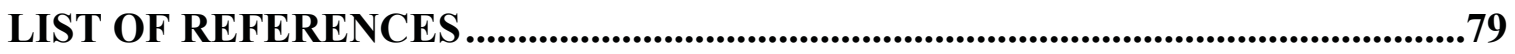

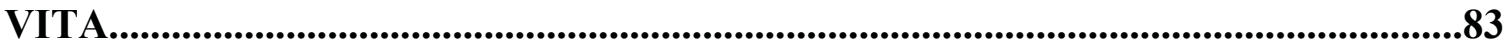




\section{LIST OF TABLES}

Table 1-1. The impact of vaccines on disease burden in the US................................2

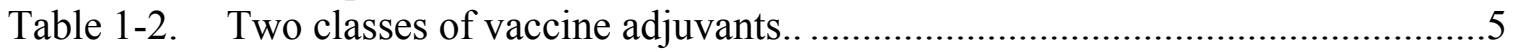

Table 1-3. Characteristics of unsuccessful and successful adjuvants.. .........................9

Table 2-1. Rabbit experimental group assignment in PZP study..............................21

Table 2-2. The results of serum anti-PZP IgG antibody titers for group 1, 2, 3, $4,5,6,7$ and 8 at different time points of day 0 , week $2,4,6,8,11$,

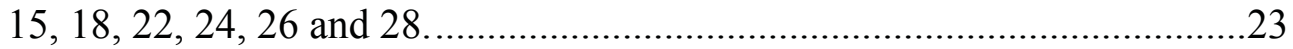

Table 2-3. Rabbit experimental group assignment in OVA study.............................27

Table 2-4. The results of serum anti-OVA IgG antibody titers for group 1, 2, 3, 4, 5, 6, 7 and 8 at different time points of day 0 , week 2, 4, 6 and 8.........29

Table 2-5. Rabbit experimental group assignment in BSA study.

Table 2-6. The results of serum anti-BSA IgG antibody titers for group 1, 2 and 3 at different time points of day 0 , week 2, 4, 6 and 8 .

Table 3-1. Mouse experimental group assignment in OVA study.

Table 3-2. Data of in vitro OVA accumulative release with four AdjuGel groups.

Table 3-3. Data of in vitro ATBC accumulative release with four AdjuGel groups.

Table 3-4. The results of serum anti-OVA IgG antibody titers for group 1, 2, 3, 4, 5, 6 and 7 at different time points of day 0 , week $2,4,6,8,11,14$ and 18

Table 3-5. The results of serum anti-OVA IgG1 antibody titers for group 1, 2, 3, 4, 5, 6 and 7 at different time points of day 0 , week 2, 4, 6, 8, 11, 14 and 18

Table 3-6. The results of serum anti-OVA IgG2a antibody titers for group 1, 2, $3,4,5,6$ and 7 at different time points of day 0 , week 2, 4, 6 and $8 \ldots \ldots \ldots . . .51$

Table 4-1. Mouse experimental group assignment.

Table 4-2. Data of in vitro OVA accumulative release with two groups.

Table 4-3. Data of in vitro ATBC accumulative release with two groups.

Table 4-4. The results of serum anti-OVA IgG antibody titers for group 1, 2, 3 and 4 at different time points of day 0 , week 2, 4, 6, 8, 10, 12, 15, 18 and 22 .

Table 5-1. Experimental group assignment with two-injections.

Table 5-2. The results of serum anti-OVA IgG antibody titers for group 1, 2, 3 and 4 at different time points of day 0 , week $2,4,6,8,10,12,15,18$ and 22. 


\section{LIST OF FIGURES}

Figure 1-1. Schematic of phase inversion process.........................................................11

Figure 1-2. Micro-CT images of a cross-section through a solidified ISI system made from 50:50 PLGA (Quenched by PBS buffer).

Figure 1-3. SEM photomicrographs of solidified ISI system formed in vivo (After 24-hours S.C. injection in mice).

Figure 1-4. Release of FITC-BSA from PLGA systems. ................................................15

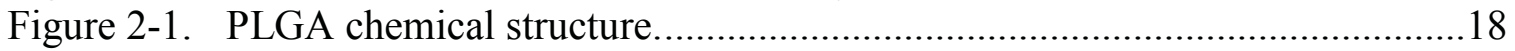

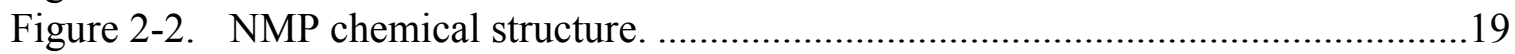

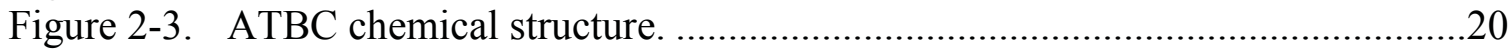

Figure 2-4. Rabbit serum anti-PZP IgG titers with multiple time points. .......................24

Figure 2-5. Rabbit serum anti-OVA IgG titers with multiple time points.......................30

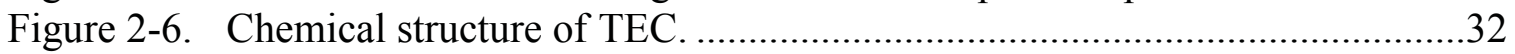

Figure 2-7. Rabbit serum anti-BSA IgG titers with multiple time points. .......................36

Figure 3-1. In vitro OVA release study with four AdjuGel groups.................................43

Figure 3-2. In vitro ATBC release study with four AdjuGel groups...............................45

Figure 3-3. Mice serum anti-OVA IgG antibody titers. ..................................................

Figure 3-4. Mice serum anti-OVA IgG1 antibody titers. .........................................50

Figure 3-5. Mice serum anti-OVA IgG2a antibody titers..........................................52

Figure 3-6. Cumulative CK-release following the injection of Nmethyl-2pyrrolidone (NMP), dimethylsulfoxide (DMSO), 2-pyrrolidone, phenytoin (positive control) and $0.9 \% \mathrm{NaCl}$ (negative control).

Figure 3-7. Cumulative CK release after 120 min following the injection of undiluted solvents in comparison to the positive control (phenytoin)

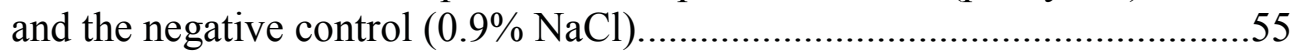

Figure 4-1. Chemical structure of poly (DL-lactide)....................................................58

Figure 4-2. Chemical structure of ethyl acetate.............................................................59

Figure 4-3. In vitro OVA accumulative release study with two groups...........................64

Figure 4-4. In vitro ATBC accumulative release study with two groups........................67

Figure 4-5. Mice serum anti-OVA IgG antibody titers. ..................................................69

Figure 5-1. Mice serum anti-OVA IgG antibody titers with two-injection group............76 


\section{LIST OF ABBREVIATIONS}

$\begin{array}{ll}\text { APCs } & \text { antigen presenting cells } \\ \text { ATBC } & \text { Acetyl Tributyl Citrate } \\ \text { BSA } & \text { bovine serum albumin } \\ \text { CTL } & \text { cytotoxic T-cell } \\ \text { DMSO } & \text { dimethylsulfoxide } \\ \text { EA } & \text { ethyl acetate } \\ \text { EIA } & \text { Enzyme immunoassay } \\ \text { ELISA } & \text { Enzyme-Linked Immunosorbant Assay } \\ \text { FCA } & \text { Freund's complete adjuvant } \\ \text { GMP } & \text { Good Manufacturing Practice } \\ \text { hCG } & \text { human chorionic gonadotropin } \\ \text { HLB } & \text { hydrophilicity lipophilicity balance } \\ \text { HPLC } & \text { high performance liquid chromatograph } \\ \text { HPV } & \text { Human papillomavirus } \\ \text { HRP } & \text { Horseradish Peroxidase } \\ \text { ISCOMS } & \text { Immune stimulating complexes } \\ \text { ISI } & \text { In Situ Implant } \\ \text { MDP } & \text { Muramyl dipeptide } \\ \text { MFA } & \text { modified Freund adjuvant } \\ \text { MPL } & \text { Monophosphoryl lipid A } \\ \text { NMP } & \text { N-Methyl-2-Pyrrolidone } \\ \text { OD } & \text { optical density } \\ \text { OVA } & \text { Ovalbumin } \\ \text { PAMPs } & \text { Pathogen-associated molecular patterns } \\ \text { PLA } & \text { Polylactic acid } \\ \text { PLGA } & \text { Poly (lactide-co-glycolide) } \\ \text { PZP } & \text { Porcine Zona Pellicida } \\ \text { RP } & \text { reverse phase } \\ \text { SA } & \text { sebacic acid } \\ \text { TEC } & \text { Triethyl Citrate } \\ & \end{array}$




\section{CHAPTER 1. INTRODUCTION}

\subsection{History of Vaccines and Vaccine Adjuvant}

\subsubsection{History of vaccines}

Immunization against smallpox, a process known as variolation started more than 1000 years ago in India and China. However, in 1789, Edward Jenner developed the first scientific smallpox vaccine, which controlled this fatal disease. Jenner's work with cowpox vaccination is widely recognized as the foundation of modern vaccinology because it's the first scientific attempt to control an infectious disease with non-diseaseleading vaccination other than transmitting the disease. [2]

Vaccination is no doubt one of the most successful methods to fight diseases in the history of human beings. Dramatic decline in the mortality and morbidity of infectious diseases is present in Table 1-1. [6]

Vaccines can be clearly divided into three general categories: live attenuated, killed and subunit vaccines.[1]

A live attenuated vaccine creates immunity by using weakened form of a living microbe with reduced virulence. Since the first vaccine, smallpox vaccine, was a live attenuated vaccine, it's not strange that live attenuated vaccines prevailed for next one hundred years. During this period, Louis Pasteur developed chicken cholera vaccine, anthrax vaccine and rabies vaccines. Live attenuated vaccines contain modified live organisms, which can cause a limited infection that induces the immune response in a similar manner to that caused by natural infection. Compared to other vaccines, live attenuated vaccines could induce the most potent and long-lasting immune responses without the help of vaccine adjuvants. However, because the organism in the live attenuated vaccines can replicate and undergo secondary mutation in the host, the vaccines may reverse to virulence and be very dangerous under some conditions of absence of immune competency.

A killed, or inactivated, vaccine is comprised of the killed whole organism. By this way, the organisms cannot replicate after administration. It was developed in the end of $19^{\text {th }}$ century. In 1896 and 1897, three killed vaccines, typhoid, cholera and plague, were invented. In the early $20^{\text {th }}$ century, more killed vaccines were developed quickly, such as pertussis vaccine, influenza vaccine and typhus vaccine. Killed vaccines cannot replicate themselves in the host, so they cannot cause persistent infection and are safer than live attenuated vaccine. However, because killed vaccines keep most of the organism virulence factors and epitopes, such as LPS and Toll like Receptors ligands, they are also very reactogenic and may induce various adverse side effects. For example, the whole cell killed pertussis vaccine caused 13,105 cases of side effects in 1979, which in turn lead to the development of a subunit pertussis vaccine to replace the killed whole 
Table 1-1. The impact of vaccines on disease burden in the US.

\begin{tabular}{lccc}
\hline Disease & $\begin{array}{c}\text { Max. no. cases } \\
\text { (year) }\end{array}$ & Cases in 2001 & $\begin{array}{c}\text { Reduction in } \\
\text { disease (\%) }\end{array}$ \\
\hline Smallpox & $48164(1901)$ & 0 & 100 \\
Diphtheria & $206939(1921)$ & 2 & 99.99 \\
Pertussis & $265269(1934)$ & 4788 & 98.2 \\
Tetanus & $1560(1923)$ & 26 & 98.34 \\
Polio & $21269(1952)$ & 0 & 100 \\
Measles & $894134(1941)$ & 96 & 99.99 \\
Rubella & $57686(1969)$ & 19 & 99.97 \\
Mumps & $152209(1968)$ & 216 & 99.86 \\
Haemophilus & $20000(1992)$ & 51 & 99.75 \\
influenzae type b & & & \\
\hline
\end{tabular}


cell vaccine.[2]

Subunit vaccines contain the acellular components like toxoid, proteins or DNA. People isolate the key components for immunization, which will protect hosts with the least side effects. Toxoids, the inactivated bacterial toxins, are firstly used for subunit vaccines. Diphtheria and Tetanus toxoids were widely applied as subunit vaccine for protection from diseases of Diphtheria and Tetanus.[2] Later, many protein and polysaccharide subunit vaccines were developed, such as vaccines for hepatitis B, influenza and meningococcal.[2] Then DNA from the pathogen was developed for new type of subunit vaccines as antigens. This type of vaccine contains no proteins or polysaccharides, but use special DNA sequence encoding a protective epitope as subunit antigens.[2] Subunit vaccines represent the development of modern vaccination with improved safety records, specially targeting the immune responses with protective epitopes and are cost effective to produce. However, since subunit vaccines contain the highly purified antigens, such as recombinant proteins, they generally lack immunogenicity and require an adjuvant and multiple doses for protection.

\subsubsection{History of vaccine adjuvants}

Adjuvant is a term derived from the Latin word adjuvare, which means to aid or to help.

In 1926, Glenny and his colleagues demonstrated the adjuvant activity of aluminum compounds with diphtheria toxoid.[2] Since then, aluminum compounds, usually referred to as "alum adjuvants", were widely used in the subunit vaccines. In 1937, Freund and his colleagues found the famous and powerful Freund's complete adjuvant (FCA), which is composed of a water in mineral oil emulsion (W/O) containing killed mycobacteria.[3] Later, it was found that killed mycobacteria are not needed in some cases and the Incomplete Freund Adjuvant was developed,[4] which is composed of a water in mineral oil emulsion without killed mycobacteria.

Generally, vaccine adjuvants are indispensable in most of the subunits vaccines. There are several important functions of vaccine adjuvants in vaccines. Firstly, vaccine adjuvant can enhance the immune response and also affect the onset and duration of immune response. Some vaccine adjuvants may induce fast and strong responses, while some adjuvants may generate delayed responses or extend the longevity of responses. Secondly, vaccine adjuvants can modify the immunophenotype or the quality of immune response that vaccines generate in the host. Different vaccines need different types of immune reaction (e.g. Th1 or Th2 type of immune response) to protect against diseases. By this function, vaccine adjuvants can determine the efficacy of the vaccines. Thirdly, vaccine adjuvants dramatically reduce the amount of antigen required to induce a protective immune response. Without adjuvants, it was found that more than ten times of antigen amount would be need to induce similar or less immune response compared to the vaccine with adjuvant. [5] Finally, vaccine adjuvants can also improve the antigens stability and extend the vaccines' shelf life. In most of the case, vaccine adjuvants may 
stabilize the vaccine antigens both in vitro and in vivo by sheltering the fragile antigens from contact with unfavorable surrounding.

Currently, many types of vaccine adjuvants have been developed to boost immune response. By mechanisms of action, Vaccine adjuvants are usually divided into two classes: delivery systems and immunopotentiators.[6] Some examples are showed in Table 1-2.[6]

\subsubsection{Alum adjuvant}

Alum is an abbreviation of aluminum salts, which include salts of aluminum hydroxide and aluminum phosphate. Alum was first used as a vaccine adjuvant in 1926 and since then, became the most widely used adjuvant. Although Alum adjuvant is a relatively weak adjuvant, it has a long safety record. For more than fifty years, alum adjuvant has been the only adjuvant in a vaccine approved for use in humans. This finally changed before the turn of the century when Novartis's (Chiron) MF59 ${ }^{\circledR}$, an oil based adjuvant, received approval for use in Europe in 1997. Twelve years later, the US FDA approved a new adjuvant ASO4 (A combined adjuvant' system containing Aluminium Hydroxide and another immune modulator called monophosphoryl lipid A), which was used in GlaxoSmithKline Biologicals's Cervarix, for prevention of cervical cancer and precancerous lesions caused by human papillomavirus (HPV) types 16 and 18.[7] Vaccines with Alum adjuvant are prepared by allowing the antigen to be absorbed to Alum. The forces responsible for the adsorption of antigen include hydrophobic interactions, van der waals forces, ionic charges and hydrogen bonding. [1]The mechanism of the adjuvanticity of Alum is not completely clear. There are three potential mechanisms to explain why Alum can induce immune response:[2]

1. The depot mechanism. The antigen is adsorbed on Alum adjuvant and is released slowly to stimulate the production of antibodies.

2. The inflammation mechanism. It is based on the hypothesis that Alum adjuvant can cause inflammation at the site of injection, which attracts antigen presenting cells (APCs), such as dendritic cells and macrophages, to process the attached antigen in the injection site.

3. Adsorption of antigen to Alum adjuvant converts the soluble antigen to a particulate form, which will facilitate APCs to take up antigen by phagocytosis. It's believed that the three mechanisms work together to provide the adjuvanticity of Alum adjuvant and further studies are still needed for elucidating the mechanisms.

\subsubsection{Oil based adjuvants}

In the various formulations of vaccine adjuvants, oil based vaccine adjuvants are among the most widely used adjuvants. Since Dr. Freund developed the famous Freund Adjuvant, many studies were conducted on the mechanisms and formulations of oil based vaccine adjuvants. Freund adjuvant was very effective, but poorly tolerated due to the use of non-degradable mineral oils, so better tolerated oils were extensively explored. In 
Table 1-2. Two classes of vaccine adjuvants.

\begin{tabular}{ll}
\hline Antigen delivery systems & Immunopotentiators \\
\hline Insoluble aluminum compounds & $\begin{array}{l}\text { MPL (Monophosphoryl lipid A) and } \\
\text { synthetic derivates }\end{array}$ \\
$\begin{array}{l}\text { Calcium phosphate } \\
\text { Liposomes }\end{array}$ & $\begin{array}{l}\text { Oligonucleotides (CpG, etc.) } \\
\text { Double-stranded RNA (dsRNA) } \\
\text { Virosomes }\end{array}$ \\
$\begin{array}{l}\text { ISCOMS } \\
\text { complexes) }\end{array}$ & $\begin{array}{l}\text { Alternative pathogen-associated molecular } \\
\text { patterns (PAMPs) (E. coli heat labile } \\
\text { enterotoxin (LT); flagellin) }\end{array}$ \\
Microparticles (e.g., poly (lactide-co- & Saponins (Quils, QS-21) \\
glycolide), PLGA) & \\
Oil-based Emulsions (e.g., IFA, MF59, & Small-molecule immune potentiators ( \\
Montanides) & SMIPs) (e.g., resiquimod [R848]) \\
Virus-like particles \& viral vectors & Cytokines \& chemokines \\
\hline
\end{tabular}


1960s, different biodegradable vegetable oils were tested in oil based adjuavnts. In 1997, MF59 was approved for use in humans. MF59 is an oil-in-water microemulsion that includes squalene (derived from biodegradable plant oil), Polysorbate 80, and Span 85(two surfactants).

The formulation of oils based vaccine adjuvant could be water in oil emulsion (e.g. IFA), oil in water emulsion (e.g. MF59) or a more complicated emulsion systems (e.g. oil in water in oil emulsion,). However, all oil based vaccine adjuvants need two necessary components in the formulation: oil and surfactant.[5] Recently, Calabro and his colleagues conducted series of experiments and demonstrated that "The adjuvant effect of MF59 is due to the oil-in-water emulsion formulation, none of the individual components induce a comparable adjuvant effect". [8] Now it is clear that only the combination of oil and surfactant can induce the adjuvant effects or immune response, any individual component, oil or surfactant alone, was not able to induce comparable immune response.

\subsubsection{Polymer vaccine adjuvants}

Polymer vaccine adjuvants have been studied for long time as vaccine delivery systems. Because polymer adjuvants have the ability of sustained release of vaccine antigens, the main purpose of polymer adjuvants is to develop single dose vaccines. In 1970 s, people started to use non-biodegradable polymer implants to develop new vaccine delivery systems. [9] Later, biodegradable polymers were introduced because biodegradable polymers didn't need to be removed by surgical procedure after administration. Since 1990s, microparticles composed of biodegradable polymers have become the most widely studied formulation as vaccine delivery systems due to the ease of administration.[10,11]

Two types of biodegradable polymers, polyesters and polyanhydrides, are most widely studied as vaccine delivery systems.

The most important polyester is poly (lactide-co-glycolide) or PLGA. PLGA was initially utilized in biomedical applications such as biodegradable surgical sutures and pins. In 1990s, PLGA microparticles were proven effective to induce protective immune responses as a vaccine delivery system. $[12,13]$ However, when PLGA degrades in vivo, lactic acid and glycolic acid are release and acidic microenvironment is created, which has been showed to be detrimental to the stability of antigen proteins. [14] In order to overcome the problem, some attempts were applied to decrease the acidity. For example, magnesium carbonate, a basic compound, was added into PLGA microspheres for stabilizing the synthetic human chorionic gonadotropin (hCG) peptide antigen.[15]

Currently, two mechanisms are available for the PLGA microparticles function as vaccine delivery system: [16] one is the PLGA microparticles form a depot for the antigen; another is PLGA microparticles enhance the APCs uptake of antigen loaded particles due to their small size. Although particle size is a key issue in the development of effective PLGA microparticles as a vaccine delivery system, the exact relationship between particle size and host immune responses is not clear. [17] There are different 
views on the relationship of particle size and the immune responses. Some believed smaller particle size intends to induce stronger immune responses, but other observations also found larger particle size might promote stronger immune responses or there was an optimum particle size that could stimulate the strongest immune response. [17]

Extensive research was done with the PLGA microparticles as antigen carriers. However, the studies still couldn't demonstrate clear correlation between release pattern of antigenic peptides, length of in vitro release of antigens, and host immune responses. [1] Because of the variation in dose of antigen, method of formulation (e.g. solvent evaporation vs. spray drying), route of administration, and the size of the microparticles, the efficacy of PLGA microparticles was not consistant in many experiments, [18] and there is no clinical trial undergoing currently with the PLGA microparticles as a vaccine delivery system. Obviously, more efforts are needed for the mechanisms and formulations of PLGA microparticles before the technique may be applied in humans.

Polyanhydrides are a class of surface erodible, biodegradable and biocompatible polymers that were widely used in controlled drug delivery systems.[19] After administration, polyanhydrides degrade into non-toxic di-acid monomers that can be metabolized and eliminated from the body. The surface erosion mechanism of polyanhydrides leads to a more predictable controlled release profile, which may range from days to months.

The most important advantage of polyanhydrides over polyesters as a vaccine delivery system is their ability to stabilize the antigen proteins. Many studies have proven that polyanhydrides are able to stably maintain polypeptides and show sustained release of polypeptides.[20, 21] The surface erosion characteristics of polyanhydrides can prevent water from penetrating into the interior of the microsphere and keep the encapsulated antigen in the native state. Moreover, the degradation products of polyanhydrides, di-acid monomers, are usually less acidic than those of PLGA polymer, which could provide a moderate $\mathrm{pH}$ microenvironment leading to stability of the encapsulated antigen and maintenance of antigenic epitopes and also reduce surrounding tissue reactions to the polymer.[22]

Despite the advantages of polyanhydrides in stabilizing the entrapped antigens, a major obstacle for the use of polyanhydride based microparticles, however, is their limited uptake by dendritic cells (DCs). In order to solve this problem, recently, Phanse et al.[23] functionalized the polyanhydride microparticle surface with di-mannose in order to target C-type lectin receptors (CLRs) on DCs. Polyanhydride particles based on sebacic acid (SA), 1,6-bis ( $p$-carboxyphenoxy)hexane (CPH) and 1,8-bis $(p$ carboxyphenoxy)-3,6-dioxaoctane (CPTEG) were evaluated. Di-mannose-functionalized polyanhydide microparticles were found to up-regulate the expression of CLRs on DCs, and more importantly, di-mannose functionalization also increased the DCs uptake of polyanhydride microparticles, which may enhance delivery of encapsulated antigens and potentially induce a more robust adaptive immune response.

Many naturally derived polymers such as chitosan, dextran, starch and alginate 
have also been explored as vaccine delivery system. [24-27] In addition, some novel synthetic polymers such as poly (ester-amide) (PEA) copolymer and poly (propylene sulfide) were studied for development of new vaccine delivery system.[1]

\subsubsection{What's the ideal vaccine adjuvant?}

In 2009, O'Hagan et al. [28] described what are the characteristics of successful and unsuccessful adjuvants.(see Table 1-3)

Vaccines should be safe and, at the same time, able to induce potent and longlasting immune responses. Because subunit vaccines usually require at least 3-5 doses to achieve protective immunity, compliance became a significant issue in vaccination programs. It's noted that dropout rates could reach $70 \%$ in some developing countries, which resulted in millions deaths annually from vaccine preventable diseases such as tetanus and pertussis.[29] In 2005, WHO listed the development of single dose vaccine as the No.1 of "Grand Challenges" in global human health. [30] [30] [30] [25] Not only in human vaccination, but in livestock systems, it's also very hard and expensive to handle the[30][30][30] large numbers of animals for multiple inoculations.[31]

In order to meet the requirement of single dose vaccines, numerous new adjuvants have been studied to approach the goal.

\subsection{Current Status for Development of Adjuvants for Single Dose Vaccine}

For subunit vaccines, there are generally two strategies to develop single dose vaccines: use a potent adjuvant (e. g. Freund complete adjuvant) or simulate multiple injections by controlled release of vaccines. Because potent adjuvants probably bring increased safety concerns, they are rarely used to design single dose vaccines. Therefore, controlled release of vaccines is mostly applied to design of single dose vaccines.

In 1979, Preis and Langer used non-biodegradable polymer implants for delivery of low dose protein antigen to mice, which showed strong and prolonged immune responses induced by sustained release of antigen.[9]

In 1991, O' Hagan et al. used biodegradable polymer (PLGA) microparticles for delivery of entrapped protein antigen to mice, which induced strong immune responses comparable to antigen dispersed in Freund's complete adjuvant.[10] PLGA is a polyester composed of lactic and/or glycolic acid monomers. PLGA has a long safety record and can be easily processed into almost any shape and size. The advantages for using PLGA microparticles as an adjuvant are: 1. controlled release of antigens for extended time periods; 2. ability to induce cytotoxic T-cell (CTL) responses,[32] which are important but absent for classic adjuvants.

Because of the advantages of PLGA microparticles for antigen delivery, PLGA 


\section{Table 1-3. Characteristics of unsuccessful and successful adjuvants.}

\begin{tabular}{|c|c|}
\hline Unsuccessful adjuvants & Successful adjuvants \\
\hline Unacceptable tolerability profile & Safe, not associated with any long term effects \\
\hline Significant local reactions & Well tolerated \\
\hline $\begin{array}{l}\text { Complex, difficult to scale up, lack of } \\
\text { reproducibility in formulation }\end{array}$ & Simple synthetic pathway \\
\hline $\begin{array}{l}\text { Raw materials expensive or not available of } \\
\text { suitable purity from reliable source }\end{array}$ & Simple inexpensive components \\
\hline $\begin{array}{l}\text { Non degradable, leaves long term residue at } \\
\text { injection sites }\end{array}$ & Biodegradable \\
\hline $\begin{array}{l}\text { Difficult to formulate with diverse antigens, } \\
\text { negative impact on antigen stability }\end{array}$ & $\begin{array}{l}\text { Compatible with many different kinds of } \\
\text { vaccine antigens }\end{array}$ \\
\hline $\begin{array}{l}\text { Inflexible, not easy to combine with additional } \\
\text { formulation components }\end{array}$ & $\begin{array}{l}\text { Capable of co-delivery of antigen and immune } \\
\text { potentiator }\end{array}$ \\
\hline
\end{tabular}


microparticles attracted extraordinary attention as a new type of vaccine adjuvant or delivery system. [33] [34] [35] However, although PLGA microparticles held many strongly desirable properties for single dose vaccines, after more than 20 years development, there are still no human clinical studies conducted with these novel vaccine delivery systems. Several significant hurdles impeded the progress of studies of PLGA microparticles as vaccines delivery systems. Such as: antigen instability when incorporated into the microparticles; high cost of manufacture process and difficulties in following the Good Manufacturing Practice (GMP) during the scale up.[16]

Although it is well known that controlled release of antigens are critical for development of new delivery systems for single dose vaccines, no clear correlation was established between release of antigens and immune response in vivo, which has become a difficukt hurdle for the further research on the new vaccine delivery system.

\subsection{What Is the ISI (In Situ Implant) System and Why Was It Chosen for Modifying Antigen Release?}

ISI systems have been developed as an alternative to solid implant and microparticle formulations.[36, 37] An ISI system is an biodegradable hydrophobic polymer (usually PLGA) solution, which is formed by dissolving biodegradable polymer into biocompatible solvents (e.g. NMP).[37] When loaded with bioactive agents by dissolved or suspended, the ISI system can be injected by syringe into the body. As biodegradable hydrophobic polymer is water insoluble, contacting with the physiologic aqueous surroundings leads to the dissipation of biocompatible solvent, causing the polymer solution to undergo phase separation to form an in situ solid or semi-solid implant.[37] The structure of solidified implant may exhibit a core with large pores of diameters from about 10 to 500 microns and a relatively nonporous skin as a membrane barrier, which has extremely fine pores of 0.01 to 0.1 microns in diameter.[38]

The solidifying process of ISI system is schematically demonstrated in the Figure 1-1. First, the homogeneous solution of PLGA polymer and solvent with drug sits on the supporter with non-solvent quenching bath surroundings, which belongs to the aqueous phase. Then solvent non-solvent exchange happens, which lead to phase inversion and PLGA precipitation. Finally, the PLGA polymer solution transforms into an implant device with a porous core and a relatively nonporous skin.[39]

Extensive studies were conducted for the ISI system as the controlled release delivery system. Krebs and his colleagues used Micro-CT to study the cross section of ISI system with PLGA polymer. [40] It is clearly showed a porous core and dense skin (Figure 1-2).

Based on the in vitro study above, Krebs, et al also studied the transformation of ISI system in vivo.[40] Figure 1-3 shows the structure of solidified ISI system formed in vivo. It also formed a porous inside and dense skin similar to Figure 1-2. 


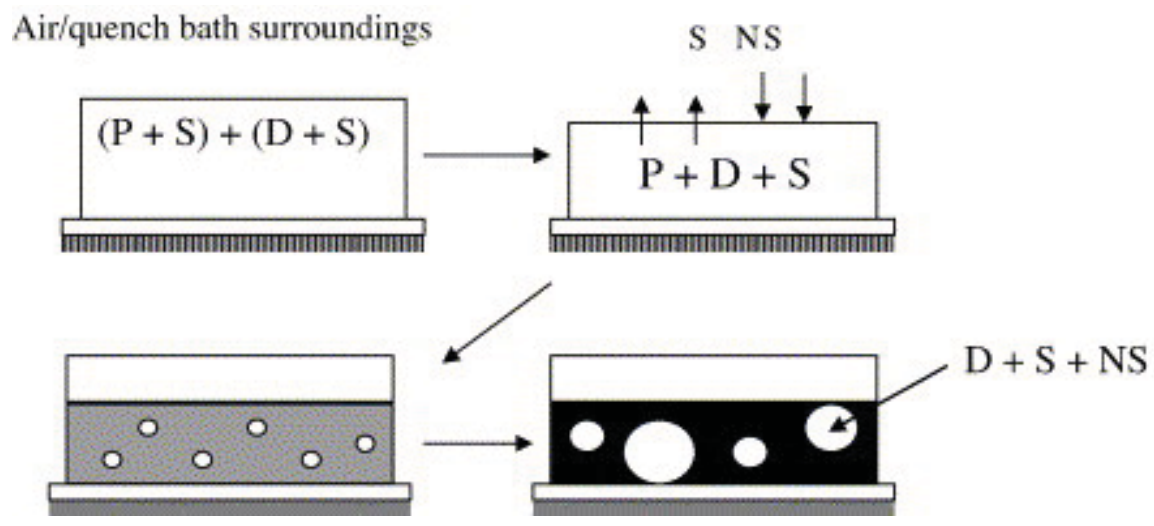

Figure 1-1. Schematic of phase inversion process.

Schematic of phase inversion process showing transformation of solution consisting of polymer (P) and solvent (S), with dissolved or suspended drug (D), to a two-phase membrane structure. (Reprinted with permission: McHugh, A.J., The role of polymer membrane formation in sustained release drug delivery systems. J Control Release, 2005. 109(1-3): p. 211-21.)

P: Polymer (e.g. PLGA); S: Solvent (e.g. N-Methyl-2-pyrrolidone, NMP); NS: Nonsolvent (e.g. water); D: Drug (e.g. Ovalbumin) 


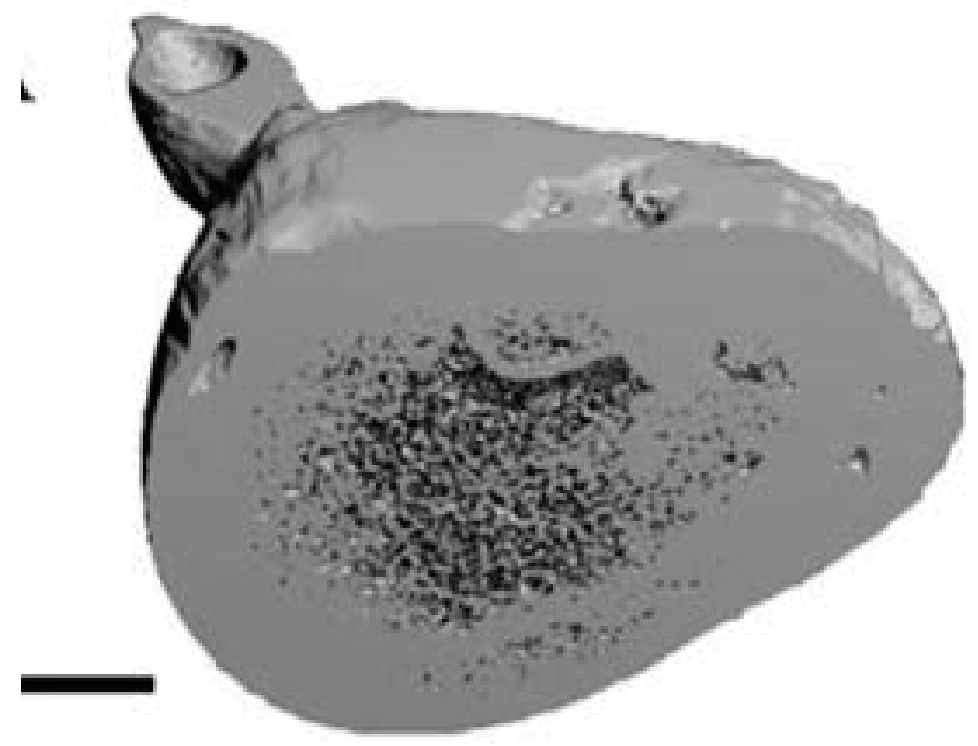

Figure 1-2. Micro-CT images of a cross-section through a solidified ISI system made from 50:50 PLGA (Quenched by PBS buffer).

Scale bars represent $1 \mathrm{~mm}$. (Reprinted with permission: Krebs, M.D., et al., Injectable poly(lactic-co-glycolic) acid scaffolds with in situ pore formation for tissue engineering. Acta Biomater, 2009. 5(8): p. 2847-59.) 

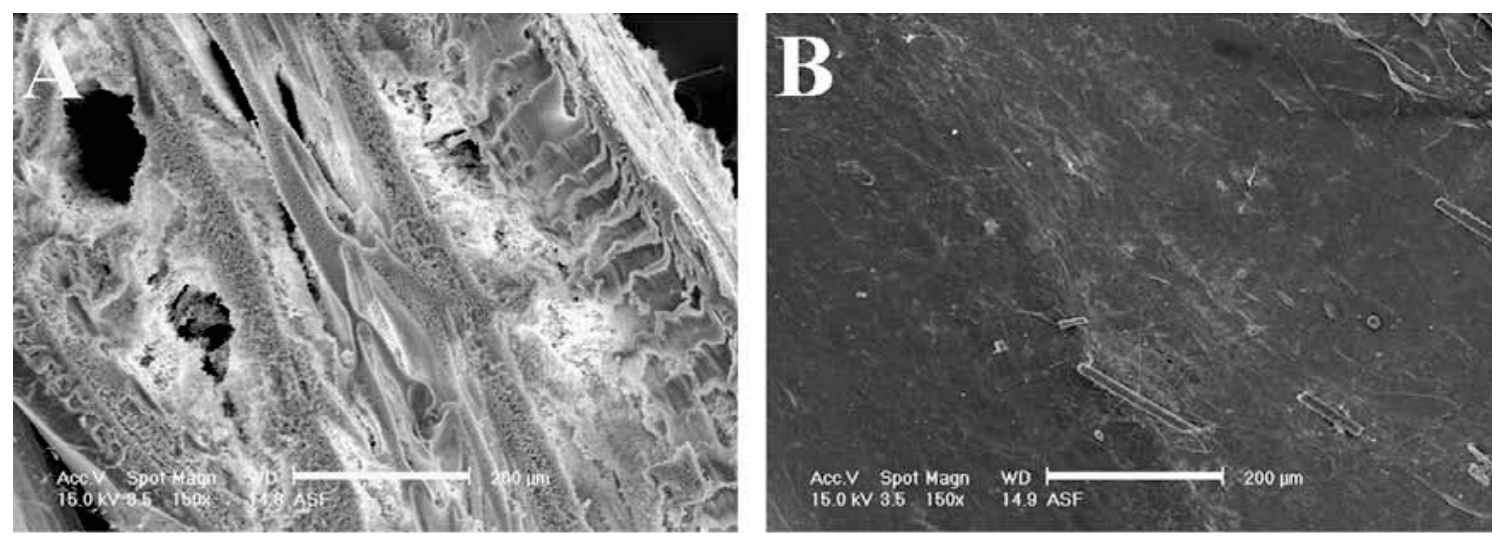

Figure 1-3. SEM photomicrographs of solidified ISI system formed in vivo (After 24-hours S.C. injection in mice).

A is from the interior of the implant, B is from the exterior of the implant. All scale bars represent 200 um. (Reprinted with permission: Krebs, M.D., et al., Injectable poly(lacticco-glycolic) acid scaffolds with in situ pore formation for tissue engineering. Acta Biomater, 2009. 5(8): p. 2847-59.) 
ISI systems were applied in the FDA approved drugs as sustained release delivery systems. Many studies were also performed to evaluate the potential of ISI system for delivery of proteins and peptides. When incorporated with lyophilized proteins, ISI system showed various sustained release profiles with the changes of different parameters (e.g. polymer types, polymer concentrations and biocompatible solvents, Figure 1-4)[41].

Although ISI system showed the potential for the control release of proteins and peptides, little data was published on the ISI system as the vaccine delivery system. in 1999, Terry Bowersock and Stephen Martin did some experiments to evaluate the possibility to use ISI system as adjuvant to develop the single dose vaccines or selfboostering vaccines.[31] They tested many formulations in pigs and finally concluded that "The profile of the immune response was similar to that seen with classical adjuvants".[31] It seemed ISI system did not exhibit special adjuvanticity even though it held the ability of prolonged release for protein antigens.

It differed from the microparticle systems, which could enhance the immune responses to antigen due to their small size, $[33,42,43]$ and the ISI system did not exhibit strong adjuvanticity and self-boostering in Bowersock's experiment. People usually believe the ISI system, based on biodegradable polymer PLGA, is an inert system, which is not suitable as vaccine delivery system. However, because the ISI system renders strong potential for the sustained release of proteins and peptide, we decided to develop ISI system as a new delivery system for single dose vaccines. 

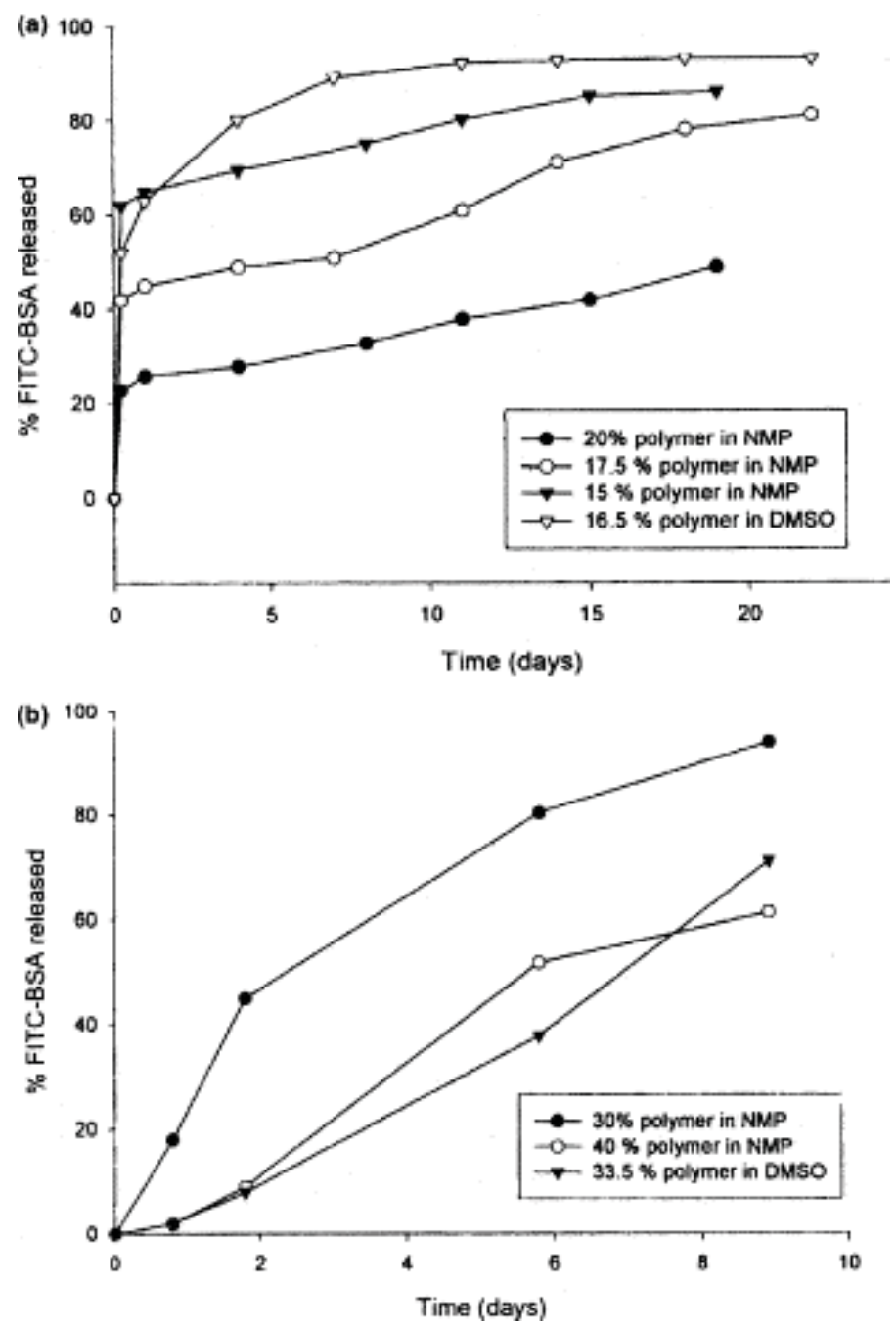

Figure 1-4. Release of FITC-BSA from PLGA systems.

(a) Release of FITC-BSA from high molecular weight PLGA systems in PBS. (b) Release of FITC-BSA from low molecular weight PLGA systems in PBS. (Reprinted with permission: Packhaeuser, C.B., et al., In situ forming parenteral drug delivery systems: an overview. Eur J Pharm Biopharm, 2004. 58(2): p. 445-55.) 


\section{CHAPTER 2. DEVELOPMENT OF NEW VACCINE FORMULATIONS WITH STRONG ADJUVANTICITY BASED ON ISI SYSTEM}

\subsection{Formulation Screening}

\subsubsection{Introduction}

Initial experiments in 2009 were initiated with the single dose Porcine Zona Pellicida (PZP) vaccine project in cooperation with Science and Conservation Center, Zoo Montana. PZP is a non-cellular membrane surrounding all mammalian eggs, which consists of three glycoproteins called ZP1, ZP2 and ZP3. PZP vaccine is a promising contraceptive vaccine under research for more than 30 years, which has been used to contracept deer, horse, elephant, wolf, sheep and many other species.[44, 45] Because PZP vaccine is mainly used for contraception of free roaming animals, multiple injections are impractical for field applications. In order to overcome the drawbacks of multiple inoculations, we designed new type of delivery system based on In Situ Implant (ISI) system to approach the goal of single inoculation.

Oil-based vaccine adjuvants, such as CFA, IFA and MF59, are probably the most effective adjuvants to stimulate immune responses. In order to enhance the adjuvanticity of vaccine delivery systems, different hydrophobic oils have already been incorporated into the microcapsule systems. [46] [47]. Because previous data [31] showed an ISI system alone could not induce strong immune response, a hydrophobic oil was considered for incorporation into an ISI system.

Mineral oil, squalene and vegetable oils were first considered as the additional components. However, mineral oil, squalene and vegetable oils were not miscible with ISI system (PLGA and organic solvent NMP solution) and they formed oil in oil emulsion after mixture. The oil in oil emulsion is not stable at room temperature and the separation happened quickly. In order to obtain a stable formulation, a hydrophobic plasticizer Acetyl Tributyl Citrate (ATBC) was introduced into ISI system. Plasticizer ATBC can be easily dissolved in the ISI system which composed of NMP and PLGA. The system with NMP, ATBC and PLGA formed a clear and stable solution formulation at room temperature.

Since Eligard $\AA$, the first FDA approved drug with ISI system, is formulated with PLGA and N-Methyl-2-pyrrolidone (NMP), we used NMP as the organic solvent in the first animal experiment. Since female rabbits are the model animals for study of PZP vaccine. New Zealand female rabbits were chose in the experiment. 


\subsubsection{Experimental procedures}

\subsubsection{Materials}

Twenty four white New Zealand female rabbits were ordered from Myrtles Rabbitry. The biodegradable polymer PLGA was ordered from Lactel Absorbable Polymers, Durect Corporation, Pelham, AL, USA. NMP was obtained from ISP Pharm Technologies; Acetyl Tributyl Citrate (ATBC) was obtained from Morflex Inc, Greensboro, NC, USA; Lyophilized PZP as the antigen was obtained from Science and Conservation Center, Zoo Montana and modified Freund adjuvant (MFA) was ordered from Sigma.

PLGA (poly (lactide-co-glycolide)) is a copolymer which is used in a lot of FDA approved therapeutic devices, owing to its biodegradability and biocompatibility. PLGA is synthesized with two different monomers, the cyclic dimers (1,4-dioxane-2,5-diones) of glycolide and lactide. Chemical structure is shown below in Figure 2-1.

NMP (N-Methyl-2-pyrrolidone) is a clear to slightly yellow liquid miscible with water and other organic solvents, which belongs to the class of dipolar aprotic solvents. Chemical structure is shown below in Figure 2-2.

Acetyl Tributyl Citrate (ATBC) is an innocuous and biodegradable plasticizer or carrier solvent permitted in the field of food additives, food contact material as well as for polymers. It was chosen as the oil because it could be easily dissolved into the NMP, PLGA solution and formed a clear formulation. Chemical structure is showed below in

Figure 2-3.

\subsubsection{Formulation process}

Formulation process of the PZP vaccine (all steps were prepared aseptically):

1. Prepare a blank gel consisting of a blend of PLGA and a combination of NMP and $\mathrm{ATBC}$ at $50^{\circ} \mathrm{C}-55^{\circ} \mathrm{C}$.

2. An appropriate quantity of gel was weighed and transferred in to an autoclaved clean glass vial. An appropriate quantity of PZP was weighed and blended with the gel and resulting mixture was stirred to obtain uniform mixture of PZP-loaded gel.

3. Each dose of PZP-loaded gel contains 100ug of PZP per $0.5 \mathrm{ml}$ gel. The final formulation is a suspension of PZP in the clear gel solution.

\subsubsection{Immunization of animals}

For all the immunization experiments, Lyophilized PZP protein was dissolved in PBS buffer or was suspended in organic solvent NMP or different gel formulations immediately before injection to obtain a concentration of $200 \mathrm{ug} / \mathrm{ml}$. All rabbits were given single IM inoculation at day 0. Experimental assignment was shown in Table 2-1. 


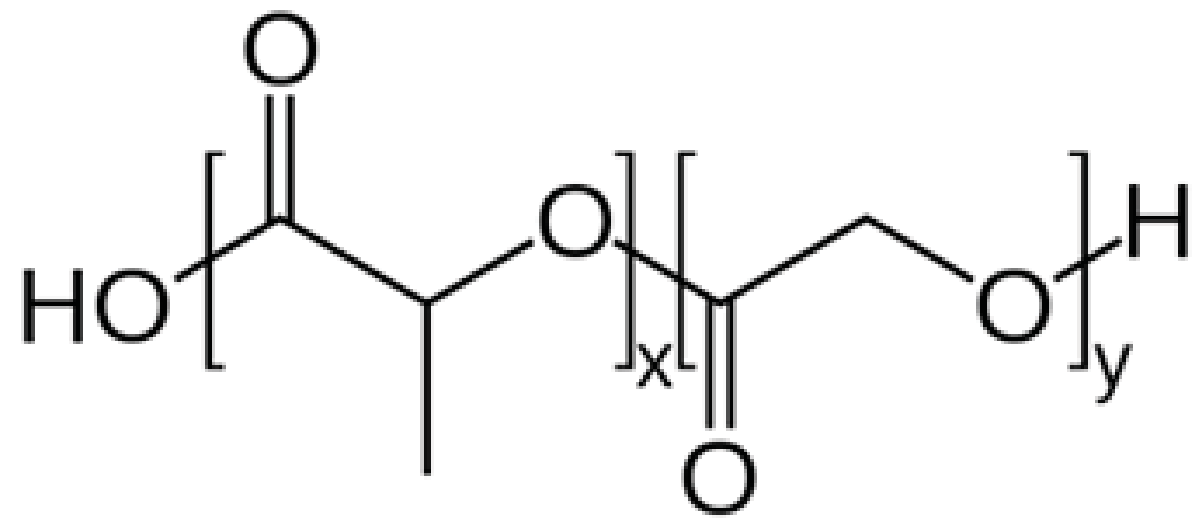

Figure 2-1. PLGA chemical structure. 


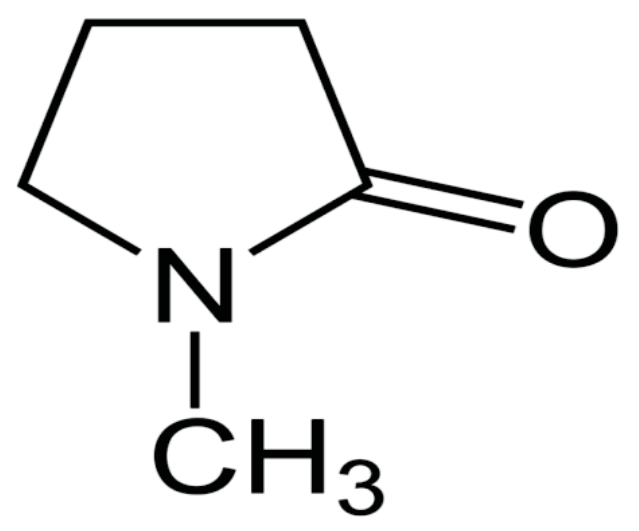

Figure 2-2. NMP chemical structure. 


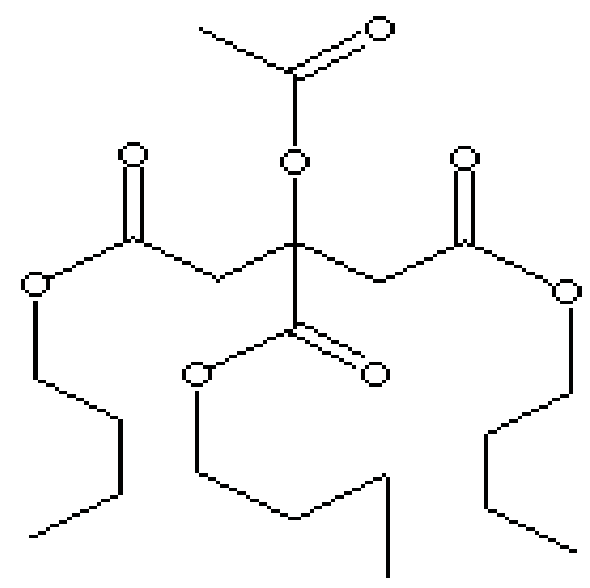

Figure 2-3. ATBC chemical structure. 
Table 2-1. Rabbit experimental group assignment in PZP study.

\begin{tabular}{|c|c|c|c|}
\hline Group & Treatment & $\begin{array}{c}\text { Number of } \\
\text { rabbits }\end{array}$ & Description \\
\hline 1 & Control & 3 & $\begin{array}{l}\text { IM injection with PBS buffer and } \\
\text { PZP (100ug for all PZP) }\end{array}$ \\
\hline 2 & NMP control & 3 & $\begin{array}{l}\text { IM injection with NMP and } \\
\text { PZP }\end{array}$ \\
\hline 3 & Modified CFA+PZP & 3 & $\begin{array}{l}\text { IM injection with Modified CFA and } \\
\text { PZP }\end{array}$ \\
\hline 4 & Gel $1+$ PZP & 3 & $\begin{array}{l}\text { IM injection with NMP plus low } \\
\text { molecular weight PLGA gel and PZP }\end{array}$ \\
\hline 5 & Gel $2+$ PZP & 3 & $\begin{array}{l}\text { IM injection with NMP plus medium } \\
\text { molecular weight PLGA gel and PZP }\end{array}$ \\
\hline 6 & Gel $3+$ PZP & 3 & $\begin{array}{l}\text { IM injection with NMP plus medium } \\
\text { molecular weight PLGA gel and PZP }\end{array}$ \\
\hline 7 & Gel 2+ ATBC + PZP & 3 & $\begin{array}{l}\text { IM injection with NMP plus low } \\
\text { molecular weight PLGA gel and PZP }\end{array}$ \\
\hline 8 & $\begin{array}{l}\text { Gel 2+ Modified CFA+ } \\
\text { PZP }\end{array}$ & 3 & $\begin{array}{l}\text { IM injection with NMP plus } \\
\text { PLGA gel and Modified CFA plus } \\
\text { PZP }\end{array}$ \\
\hline
\end{tabular}

The final volume of each group is $0.5 \mathrm{ml}$.

The PLGA polymer in Group 5, 7 and 8 is medium molecular weight PLGA (50:50) (IV: 0.55 - 0.75; product No. B6010-2, Durect Corporation).

The PLGA polymer in Group 4 is the low molecular weight PLGA 50:50 (IV: 0.15 0.25; Product No. B6017-1, Durect Corporation)

The PLGA polymer in Group 6 is high molecular weight PLGA 85:15 (IV: 0.55 - 0.75;

Product No. B6006-1, Durect Corporation) 


\subsubsection{Procedures}

All groups were inoculated IM once at day 0 and blood samples were collected every 2 weeks in the first 2 months and every 3 or 4 weeks thereafter. Blood samples were serially diluted with $0.05 \%$ Tween 20 in PBS: 1:1,000, 1:10,000, 1:100,000 and 1:1,000,000. IgG titers were checked by ELISA (Enzyme-Linked Immunosorbant Assay); use Log10EC50 as a measure of immune response. EC50 means half maximal effective concentration and the absorbance of 1:1000 dilution was used as the maximum.

\subsubsection{Enzyme linked immunosorbent assay (ELISA)}

96-well microtiter plates (high protein binding plates from Costar) were coated overnight with $100 \mu \mathrm{l}$ coating solution containing $3 \mu \mathrm{g} / \mathrm{mL}$ PZP protein at $4{ }^{\circ} \mathrm{C}$. To remove unbound PZP, plates were washed three times with PBS ( $\mathrm{pH} 7.4)$ containing $0.05 \%$ Tween 20 (PBST). Serum samples $(100 \mu \mathrm{L} /$ well) from individual mice were serially diluted in PBST: 1:1,000, 1:10,000, 1:100,000 and 1:1,000,000. The plates were then incubated for two hours at room temperature. The plates were again washed three times with PBST followed by addition of $100 \mu \mathrm{L}$ of PBST containing Horseradish Peroxidase (HRP) -conjugated goat anti-rabbit IgG (diluted 1:4000) (Abcam, ab6721). After a two hour incubation period at room temperature, the plates were washed three times with PBST followed by the addition of $200 \mu \mathrm{L}$ of $0.4 \mathrm{mg} / \mathrm{ml}$ OPD Peroxidase Substrate (Sigma P9187, Sigma-Aldrich, St. Louis, MO) and allowed to react for 30 minutes at room temperature. The optical density (OD) of the reaction was measured at $450 \mathrm{~nm}$ using a Plate Reader. Software BioDataFit was applied to deal with the data by the four-parameter model. Serum titers are reported with Log10EC50.

\subsubsection{Statistical analysis}

Results are expressed as mean \pm S.D. Statistical analysis was carried out for analyses of differences between means of serum antibody titers using Student's twotailed $t$-test. Differences in means were accepted as significant if $P$ was less than 0.05 .

\subsubsection{Results and discussion}

Serum titers were reported with $\log 10 \mathrm{EC} 50$ and results was showed in Table 2-2 and Figure 2-4. Table 2-2 showed the means of serum anti-PZP IgG antibody titers and standard deviations.

In this experiment, the formulations with only organic solvent NMP and three different types of PLGA (Group 4, 5 and 6) didn't induce strong immune response. At week 6, there are no significant differences among the negative control (group 1), group 4, 5 and $6(\mathrm{P}>0.05)$. However, when incorporated with ATBC and modified Freund adjuvant (mineral oil) in group 7 and 8 , they both exhibited much stronger immune response. At week 6, there are significant differences between ATBC group (group 7) and non-ATBC group (group 4) $(\mathrm{P}<0.05)$. Since the formulation of group 8 formed an 
Table 2-2. The results of serum anti-PZP IgG antibody titers for group 1, 2, 3, 4, 5, 6, 7 and 8 at different time points of day 0 , week $2,4,6,8,11,15,18,22,24,26$, and 28 .

\begin{tabular}{lcccccccc}
\hline Time & G1 & G2 & G3 & G4 & G5 & G6 & G7 & G8 \\
\hline day 0 & $0 \pm 0$ & $0 \pm 0$ & $0 \pm 0$ & $0 \pm 0$ & $0 \pm 0$ & $0 \pm 0$ & $0 \pm 0$ & $0 \pm 0$ \\
2 weeks & $0.4 \pm 0.16$ & $0.48 \pm 0.07$ & $1 \pm 0.5$ & $0.59 \pm 0.2$ & $0.59 \pm 0.1$ & $0.29 \pm 0.06$ & $0.9 \pm 0.28$ & $0.69 \pm 0.23$ \\
4 weeks & $0.44 \pm 0.14$ & $0.34 \pm 0.1$ & $1.58 \pm 0.33$ & $0.62 \pm 0.07$ & $0.86 \pm 0.04$ & $0.36 \pm 0.17$ & $1.19 \pm 0.23$ & $1 \pm 0.17$ \\
6 weeks & $0.63 \pm 0.05$ & $0.31 \pm 0.1$ & $2.34 \pm 0.15$ & $0.78 \pm 0.09$ & $0.81 \pm 0.18$ & $0.52 \pm 0.27$ & $1.31 \pm 0.19$ & $1.35 \pm 0.18$ \\
8 weeks & $0.58 \pm 0.14$ & $0.2 \pm 0.09$ & $2.39 \pm 0.48$ & $0.7 \pm 0.1$ & $0.53 \pm 0.12$ & $0.42 \pm 0.41$ & $1.18 \pm 0.43$ & $1.49 \pm 0.41$ \\
11 weeks & $0.32 \pm 0.11$ & $0.19 \pm 0.16$ & $2.36 \pm 0.53$ & $0.32 \pm 0.13$ & $0.54 \pm 0.21$ & $0.22 \pm 0.21$ & $0.71 \pm 0.23$ & $1.44 \pm 0.36$ \\
15 weeks & $0.28 \pm 0.12$ & $0.1 \pm 0$ & $2.13 \pm 0.43$ & $0.1 \pm 0$ & $0.13 \pm 0.05$ & $0.22 \pm 0.18$ & $0.87 \pm 0.32$ & $1.49 \pm 0.41$ \\
18 weeks & $0.16 \pm 0.11$ & $0.1 \pm 0$ & $2.05 \pm 0.45$ & $0.12 \pm 0.03$ & $0.19 \pm 0.1$ & $0.15 \pm 0.08$ & $0.48 \pm 0.13$ & $1.15 \pm 0.4$ \\
22 weeks & $0.16 \pm 0.05$ & $0.1 \pm 0$ & $2.14 \pm 0.58$ & $0.1 \pm 0$ & $0.16 \pm 0.1$ & $0.13 \pm 0.06$ & $0.51 \pm 0.33$ & $1.41 \pm 0.39$ \\
24 weeks & $0.13 \pm 0.06$ & $0.1 \pm 0$ & $2.03 \pm 0.57$ & $0.1 \pm 0$ & $0.15 \pm 0.09$ & $0.12 \pm 0.03$ & $0.36 \pm 0.14$ & $1.21 \pm 0.5$ \\
26 weeks & $0.1 \pm 0.06$ & $0.1 \pm 0$ & $1.95 \pm 0.76$ & $0.1 \pm 0$ & $0.1 \pm 0$ & $0.1 \pm 0$ & $0.39 \pm 0.24$ & $0.94 \pm 0.49$ \\
28 weeks & $0.1 \pm 0.06$ & $0.1 \pm 0$ & $1.71 \pm 0.55$ & $0.1 \pm 0$ & $0.1 \pm 0$ & $0.1 \pm 0.03$ & $0.26 \pm 0.1$ & $0.98 \pm 0.52$ \\
P value & & & & & & & \\
(6 weeks) & & & & $0.075^{*}$ & $0.202 * *$ & $0.549 * * *$ & $0.030^{* * * *}$
\end{tabular}

Serum anti-PZP IgG antibody titers were reported with Log10EC50 and results were presented as Mean \pm S.D.

P values with 6 weeks results (*G4 vs. G1; **G5 vs. G1; ***G6 vs. G1; ****G5 vs. G7) 


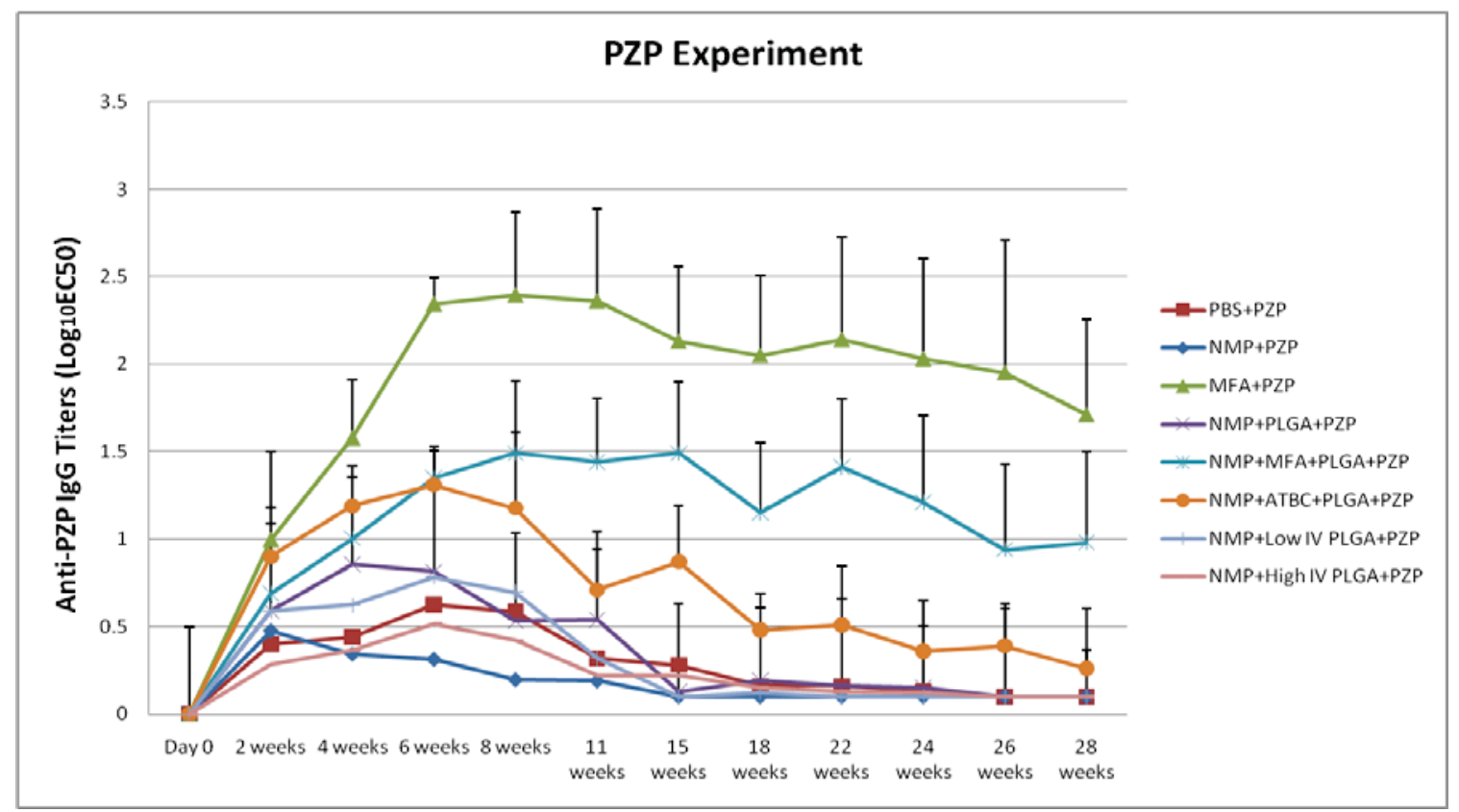

Figure 2-4. Rabbit serum anti-PZP IgG titers with multiple time points. 
oil in oil emulsion and the emulsion was not stable, we didn't conduct further studies for this formulation. The formulation with organic solvent NMP, PLGA and ATBC was a stable and clear solution, which we chose as the candidate for further development.

\subsubsection{Conclusions}

From the results we got, we concluded that: 1. The gel comprised of NMP and PLGA (ISI system) didn't show good adjuvanticity, which is compatible with previously published data. 2. The gel comprised of NMP, PLGA and ATBC (called AdjuGel system) can induce strong immune responses and it is a new type of vaccine adjuvant or delivery system which has potent ability for the sustained release of both antigens and vaccine adjuvants. 3. The gel comprised of NMP, PLGA and ATBC (called AdjuGel system) demonstrated potential as a long acting system for administration of PZP for contraception.

\subsection{Study the AdjuGel System and Determine If PLGA Is Necessary for Adjuvanticity}

\subsubsection{Introduction}

Because AdjuGel system is a new type of vaccine adjuvant, we need to study this new formulation to clarify what's the key component for its adjuvanticity. In previous experiment, we obtained the result that organic solvent NMP alone (group 2) cannot induce an effective immune response. However, NMP is well known as a chemical penetration enhancer, [48] and is widely used for enhancement of transdermal delivery of different hydrophilic and hydrophobic drugs.[49] NMP can also induce the local inflammation after IM injection. [50] We assumed NMP may play a role in the adjuvanticity of AdjuGel system. PLGA polymers have been widely utilized in biomedical applications such as therapeutic devices and biodegradable surgical sutures due to their biodegradability and biocompatibility. It is well accepted that PLGA polymers are inert and cause little local inflammation. Based on the characteristics of NMP and PLGA polymers, we set up a new animal experiment to answer several questions: 1. is biodegradable polymer PLGA indispensable for the adjuvanticity? 2. may ATBC alone good enough for adjuvanticity? 3. is NMP plus ATBC a good formulation of vaccine adjuvant?

\subsubsection{Experimental procedures}

\subsubsection{Materials}

24 white New Zealand female rabbits were ordered from Myrtles Rabbitry. The biodegradable polymer PLGA was ordered from Lactel Absorbable Polymers, Durect 
Corporation, Pelham, AL, USA. NMP was obtained from ISP Pharm Technologies; Acetyl Tributyl Citrate (ATBC) was obtained from Morflex Inc, Greensboro, NC, USA; Lyophilized Ovalbumin (OVA) as the antigen was purchased from Sigma; Alum was ordered from Thermo Scientific.

\subsubsection{Formulation process}

Formulation process of the OVA vaccine (all steps were prepared aseptically): 1. Prepare a blank gel consisting of a blend of PLGA and a combination of NMP and ATBC at $50^{\circ} \mathrm{C}-55^{\circ} \mathrm{C}$. 2. An appropriate quantity of gel was weighed and transferred in to an autoclaved clean glass vial. An appropriate quantity of OVA was weighed and blended with the gel and resulting mixture was stirred to obtain uniform mixture of OVA-loaded gel. 3. Each dose of OVA-loaded gel contains 100 ug of OVA per $0.5 \mathrm{ml}$ gel. The final formulation is a suspension of OVA in the clear gel solution.

\subsubsection{Immunization of animals}

For all the immunization experiments, Lyophilized OVA protein was dissolved in PBS buffer or was suspended in organic solvent NMP, plasticizer ATBC or AdjuGel system immediately before injection to obtain a concentration of $200 \mathrm{ug} / \mathrm{ml}$. Experimental assignment was shown in Table 2-3.

\subsubsection{Procedures}

All groups were inoculated IM once at day 0 and blood samples were collected every 2 weeks. Blood samples were serially diluted with $0.05 \%$ Tween 20 in PBS: $1: 1,000,1: 10,000,1: 100,000$ and 1:1,000,000. IgG titers were checked by ELISA (Enzyme-Linked Immunosorbant Assay); use Log10EC50 as a measure of immune response. EC50 means half maximal effective concentration and the absorbance of 1:1000 dilution was used as the maximum.

\subsubsection{Enzyme linked immunosorbent assay (ELISA)}

96-well microtiter plates (high protein binding plates from Costar) were coated overnight with $100 \mu \mathrm{l}$ coating solution containing $3 \mu \mathrm{g} / \mathrm{mL}$ ovalbumin (OVA) at $4{ }^{\circ} \mathrm{C}$. To remove unbound OVA, plates were washed three times with PBS (pH 7.4) containing $0.05 \%$ Tween 20 (PBST). Serum samples $(100 \mu \mathrm{L} /$ well) from individual mice were serially diluted in PBST: 1:1,000, 1:10,000, 1:100,000 and 1:1,000,000. The plates were then incubated for two hours at room temperature. The plates were again washed three times with PBST followed by addition of $100 \mu \mathrm{L}$ of PBST containing Horseradish Peroxidase (HRP) -conjugated goat anti-rabbit IgG (diluted 1:4000) (Abcam, ab6721). After a two hour incubation period at room temperature, the plates were washed three times with PBST followed by the addition of $200 \mu \mathrm{L}$ of $0.4 \mathrm{mg} / \mathrm{ml}$ OPD Peroxidase Substrate (Sigma P9187, Sigma-Aldrich, St. Louis, MO) and allowed to react for 30 minutes at room temperature. The optical density (OD) of the reaction was measured at $450 \mathrm{~nm}$ using a Plate Reader. Software BioDataFit was applied to deal with the data by 
Table 2-3. Rabbit experimental group assignment in OVA study.

\begin{tabular}{llcl}
\hline Group & Treatment & $\begin{array}{c}\text { Number } \\
\text { of } \\
\text { rabbits }\end{array}$ & Description \\
\hline 1 & Control & 3 & IM injection with PBS buffer and OVA \\
2 & NMP control & 3 & IM injection with NMP and OVA \\
3 & ALUM+OVA & 3 & IM injection with ALUM and OVA \\
4 & ATBC + OVA & 3 & IM injection with ATBC and OVA \\
5 & ATBC+NMP+OVA & 3 & IM injection with ATBC and NMP $(2: 1 \mathrm{~V} / \mathrm{V})$ plus OVA \\
6 & ATBC+NMP+OVA & 3 & IM injection with ATBC and NMP $(1: 1 \mathrm{~V} / \mathrm{V})$ plus OVA \\
7 & ATBC+NMP+OVA & 3 & IM injection with ATBC and NMP $(1: 2 \mathrm{~V} / \mathrm{V})$ plus OVA \\
8 & AdjuGel + OVA & 3 & IM injection with ATBC and NMP $(1: 1 \mathrm{~V} / \mathrm{V})$ plus PLGA and \\
& & & OVA \\
\hline
\end{tabular}

Ovalbumin is $100 \mathrm{ug}$ per injection. The volume is $0.5 \mathrm{ml}$ per injection. 
the four-parameter model. Serum titers are reported with Log10EC50.

\subsubsection{Statistical analysis}

Results are expressed as mean \pm S.D. Statistical analysis was carried out for analyses of differences between means of serum antibody titers using Student's twotailed $t$-test. Differences in means were accepted as significant if $P$ was less than 0.05 .

\subsubsection{Results and discussion}

Serum titers were reported in Table 2-4 and Figure 2-5.

Biodegradable hydrophobic polymers PLGA are thought to be inert materials, so we assumed the removal of PLGA would not affect the adjuvanticity of AdjuGel. However, none of the groups (group 4, 5, 6 and 7) without PLGA exhibited adjuvanticity. AT time points of week 4, 6 and 8, the group 8, composed of NMP, PLGA and ATBC, induced a much stronger immune response than the Alum group $(\mathrm{P}<0.05)$. Clearly, polymer PLGA played a key role in the AdjuGel as a vaccine adjuvant.

\subsubsection{Conclusions}

From the study and results above, we concluded that: 1. In the AdjuGel formula of NMP, PLGA and ATBC, the PLGA polymer as well as ATBC is indispensable for the adjuvanticity. 2. The AdjuGel system induced a much stronger immune response in comparison with Alum $(\mathrm{P}<0.05)$.

\subsection{Study the AdjuGel System and Determine If NMP Is Necessary for Adjuvanticity}

\subsubsection{Introduction}

In the previous studies, we demonstrated that ATBC and PLGA are indispensible in the AdjuGel system as a vaccine adjuvant. Now we want to answer the question: is the organic solvent NMP an essential component in the AdjuGel system for adjuvanticity. The organic solvent NMP has been used in some of FDA approved drugs, but it belongs to the class of dipolar aprotic solvents and may induce acute myotoxicity and local inflammation,[51] which may help in the adjuvanticity of the Adjugel system. In the new experiment, we used Triethyl Citrate (TEC), a relatively more hydrophobic liquid, to replace NMP as solvent in AdjuGel system. 
Table 2-4. The results of serum anti-OVA IgG antibody titers for group 1, 2, 3, 4, 5, 6, 7 and 8 at different time points of day 0 , week 2, 4, 6 and 8 .

\begin{tabular}{lcccccccc}
\hline Time & G1 & G2 & G3 & G4 & G5 & G6 & G7 & G8 \\
\hline Day 0 & $0 \pm 0$ & $0 \pm 0$ & $0 \pm 0$ & $0 \pm 0$ & $0 \pm 0$ & $0 \pm 0$ & $0 \pm 0$ & $0 \pm 0$ \\
2 weeks & $0.34 \pm 0.42$ & $0.1 \pm 0$ & $0.67 \pm 0.43$ & $0.1 \pm 0$ & $0.34 \pm 0.42$ & $0.26 \pm 0.28$ & $0.1 \pm 0$ & $0.74 \pm 0.42$ \\
4 weeks & $0.31 \pm 0.37$ & $0.1 \pm 0$ & $0.69 \pm 0.33$ & $0.1 \pm 0$ & $0.31 \pm 0.37$ & $0.29 \pm 0.33$ & $0.1 \pm 0$ & $1.41 \pm 0.19$ \\
6 weeks & $0.17 \pm 0.12$ & $0.1 \pm 0$ & $0.55 \pm 0.35$ & $0.1 \pm 0$ & $0.17 \pm 0.12$ & $0.24 \pm 0.24$ & $0.1 \pm 0$ & $1.48 \pm 0.22$ \\
8 weeks & $0.27 \pm 0.29$ & $0.1 \pm 0$ & $0.63 \pm 0.53$ & $0.1 \pm 0$ & $0.27 \pm 0.29$ & $0.16 \pm 0.1$ & $0.1 \pm 0$ & $1.29 \pm 0.25$ \\
P value & & & & & & & & \\
(6 weeks) & & & & & & & $0.025^{*}$ \\
\hline
\end{tabular}

Serum anti-OVA IgG antibody titers were reported with $\log 10 \mathrm{EC} 50$ and results were presented as Mean \pm S.D.

P value with 6 weeks results (*G3 vs. G8) 


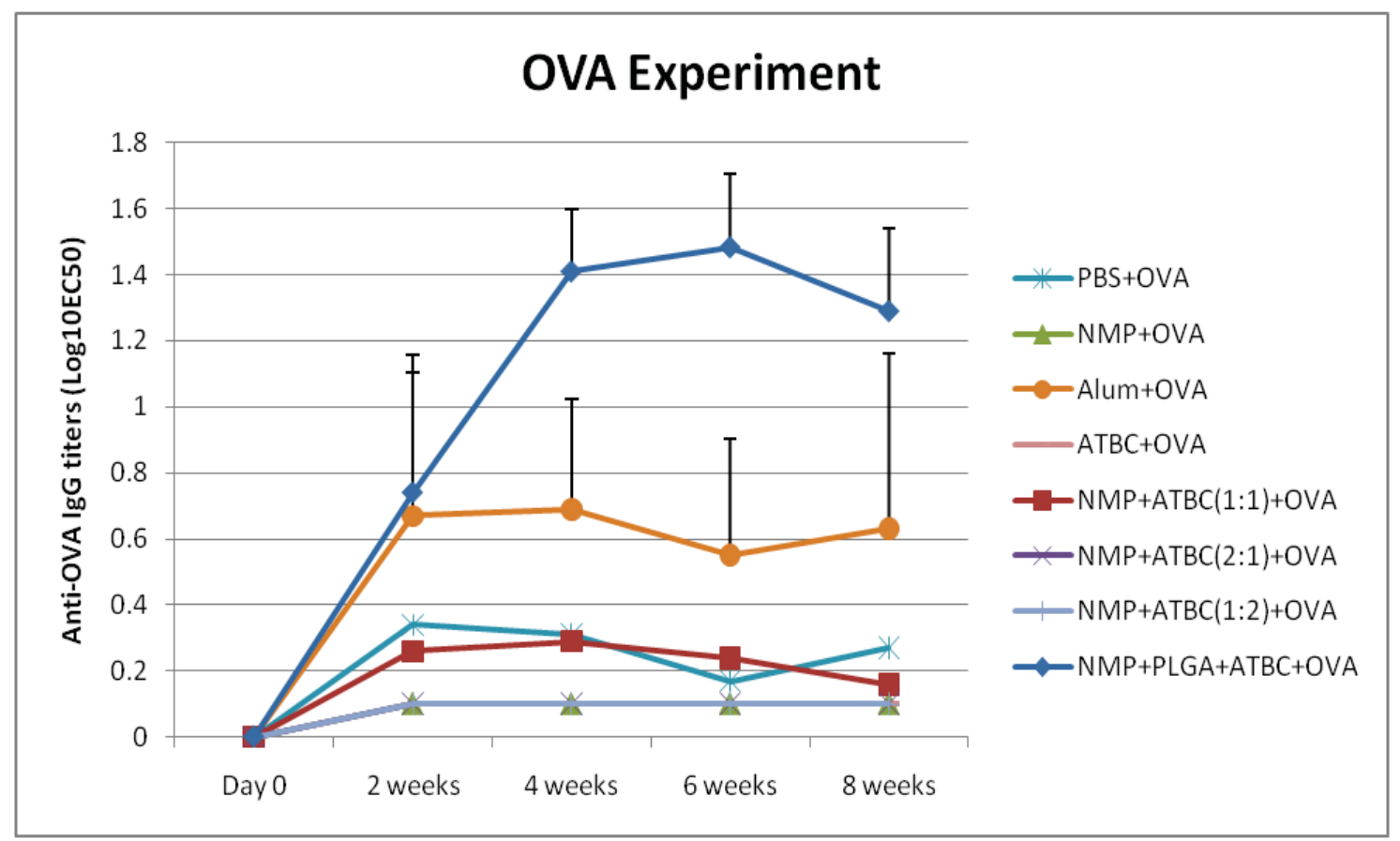

Figure 2-5. Rabbit serum anti-OVA IgG titers with multiple time points. 


\subsubsection{Experimental procedures}

\subsubsection{Materials}

Nine white New Zealand female rabbits were ordered from Myrtles Rabbitry. The biodegradable polymer PLGA was ordered from Lactel Absorbable Polymers, Durect Corporation, Pelham, AL, USA. Triethyl Citrate (TEC) was obtained from Morflex Inc, Greensboro, NC, USA; Lyophilized bovine serum albumin (BSA) as the antigen was purchased from Sigma; Alum was ordered from Thermo Scientific. Triethyl Citrate (TEC) is a colorless, odorless oily liquid, which is relatively more hydrophobic than NMP. In this experiment, we used TEC to replace NMP as organic solvent. The chemical structure of TEC is showed below (Figure 2-6).

\subsubsection{Formulation process}

Formulation process of the BSA vaccine (all steps were prepared aseptically): 1 . Prepare a blank gel consisting of a blend of PLGA and a combination of TEC and ATBC at $50^{\circ} \mathrm{C}-55^{\circ} \mathrm{C}$. 2. An appropriate quantity of gel was weighed and transferred in to an autoclaved clean glass vial. An appropriate quantity of BSA was weighed and blended with the gel and resulting mixture was stirred to obtain uniform mixture of BSA-loaded gel. 3. Each dose of BSA-loaded gel contains 100ug of BSA per $0.5 \mathrm{ml}$ gel. The final formulation is a suspension of BSA in the clear gel solution.

\subsubsection{Immunization of animals}

For all the immunization experiments, Lyophilized BSA protein was dissolved in PBS buffer or was suspended in AdjuGel system with organic solvent TEC immediately before injection to obtain a concentration of $200 \mathrm{ug} / \mathrm{ml}$. All rabbits were given single IM inoculation at day 0. Experimental assignment was shown in Table 2-5.

\subsubsection{Procedure}

All groups were inoculated IM once at day 0 and blood samples were collected every 2 weeks. Blood samples were serially diluted with $0.05 \%$ Tween 20 in PBS: $1: 1,000,1: 10,000,1: 100,000$ and 1:1,000,000. IgG titers were checked by ELISA (Enzyme-Linked Immunosorbant Assay); use Log10EC50 as a measure of immune response. EC50 means half maximal effective concentration and the absorbance of 1:1000 dilution was used as the maximum.

\subsubsection{Enzyme linked immunosorbent assay (ELISA)}

96-well microtiter plates (high protein binding plates from Costar) were coated overnight with $100 \mu \mathrm{l}$ coating solution containing $3 \mu \mathrm{g} / \mathrm{mL}$ Bovine Serum Albumin (BSA) at $4{ }^{\circ} \mathrm{C}$. To remove unbound BSA, plates were washed three times with PBS (pH 7.4) containing $0.05 \%$ Tween 20 (PBST). Serum samples $(100 \mu \mathrm{L} /$ well $)$ from individual rabbit were serially diluted in PBST: 1:1,000, 1:10,000, 1:100,000 and 1:1,000,000. The 


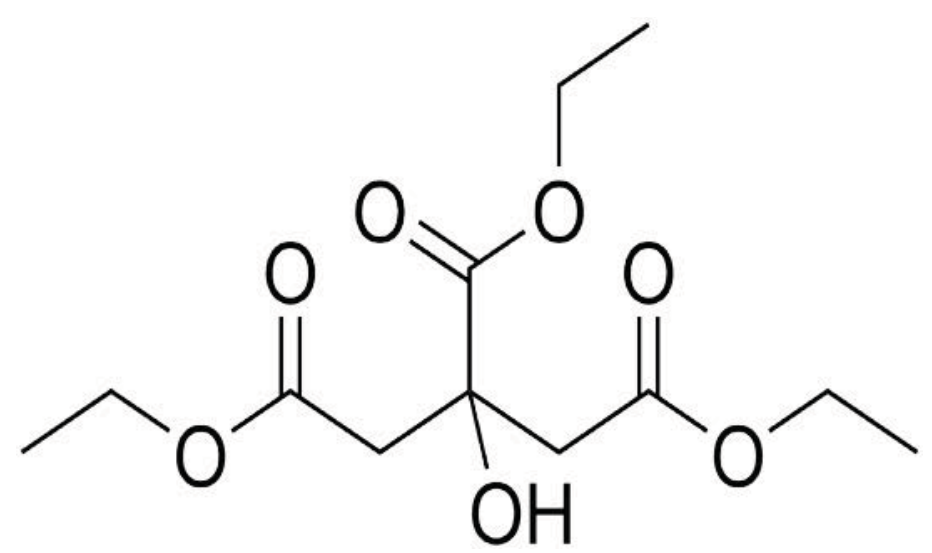

Figure 2-6. Chemical structure of TEC. 
Table 2-5. Rabbit experimental group assignment in BSA study.

\begin{tabular}{clcl}
\hline Group & Treatment & $\begin{array}{c}\text { Number of } \\
\text { rabbits }\end{array}$ & Description \\
\hline 1 & Control & 3 & IM injection with PBS buffer and \\
2 & ALUM+BSA & 3 & BSA \\
3 & TEC+PLGA+ATBC+BSA & 3 & $\begin{array}{l}\text { IM injection with ALUM and BSA } \\
(2: 1 \mathrm{~V} / \mathrm{V}) \text { plus PLGA and BSA }\end{array}$ \\
\hline
\end{tabular}

TEC (Triethyl Citrate) is a relatively more hydrophobic solvent when compared with NMP. BSA is $100 \mathrm{ug}$ per injection. The volume is $0.5 \mathrm{ml}$ per injection. 
plates were then incubated for two hours at room temperature. The plates were again washed three times with PBST followed by addition of $100 \mu \mathrm{L}$ of PBST containing Horseradish Peroxidase (HRP) -conjugated goat anti-rabbit IgG (diluted 1:4000) (Abcam, ab6721). After a two hour incubation period at room temperature, the plates were washed three times with PBST followed by the addition of $200 \mu \mathrm{L}$ of $0.4 \mathrm{mg} / \mathrm{ml}$ OPD Peroxidase Substrate (Sigma P9187, Sigma-Aldrich, St. Louis, MO) and allowed to react for 30 minutes at room temperature. The optical density (OD) of the reaction was measured at $450 \mathrm{~nm}$ using a Plate Reader. Software BioDataFit was applied to deal with the data by the four-parameter model. Serum titers are reported with Log10EC50.

\subsubsection{Statistical analysis}

Results are expressed as mean \pm S.D. Statistical analysis was carried out for analyses of differences between means of serum antibody titers using Student's twotailed $t$-test. Differences in means were accepted as significant if $P$ was less than 0.05 .

\subsubsection{Results and discussion}

Serum titers were reported with Log10EC50 and results was showed in Table 2-6 and Figure 2-7.

From the results we could see that even we used a more hydrophobic organic solvent, instead of NMP, the AdjuGel system can still induce strong immune response. At time point of week 4, there is significant difference between group 3, composed of TEC, PLGA and ATBC, and group 1(negative control) $(\mathrm{P}<0.05)$.

\subsubsection{Conclusion}

In the AdjuGel system, organic solvent NMP is replaceable and other more hydrophobic solvents could be used in this system. 
Table 2-6. The results of serum anti-BSA IgG antibody titers for group 1, 2 and 3 at different time points of day 0 , week $2,4,6$ and 8 .

\begin{tabular}{lccc}
\hline Time & G1 & G2 & G3 \\
\hline Day 0 & $0 \pm 0$ & $0 \pm 0$ & $0 \pm 0$ \\
2 weeks & $0.91 \pm 0.7$ & $1.42 \pm 0.25$ & $0.92 \pm 0.38$ \\
4 weeks & $0.64 \pm 0.49$ & $1.18 \pm 0.04$ & $1.68 \pm 0.32$ \\
6 weeks & $0.78 \pm 0.59$ & $1.27 \pm 0.21$ & $1.58 \pm 0.26$ \\
8 weeks & $0.48 \pm 0.42$ & $0.96 \pm 0.22$ & $1.36 \pm 0.34$ \\
P value & & & $0.044^{*}$ \\
(4 weeks) & & & \\
\hline
\end{tabular}

Serum anti-BSA IgG antibody titers were reported with Log10EC50 and results were presented as Mean \pm S.D.

P value with 4 weeks results (*G3 vs. G1) 


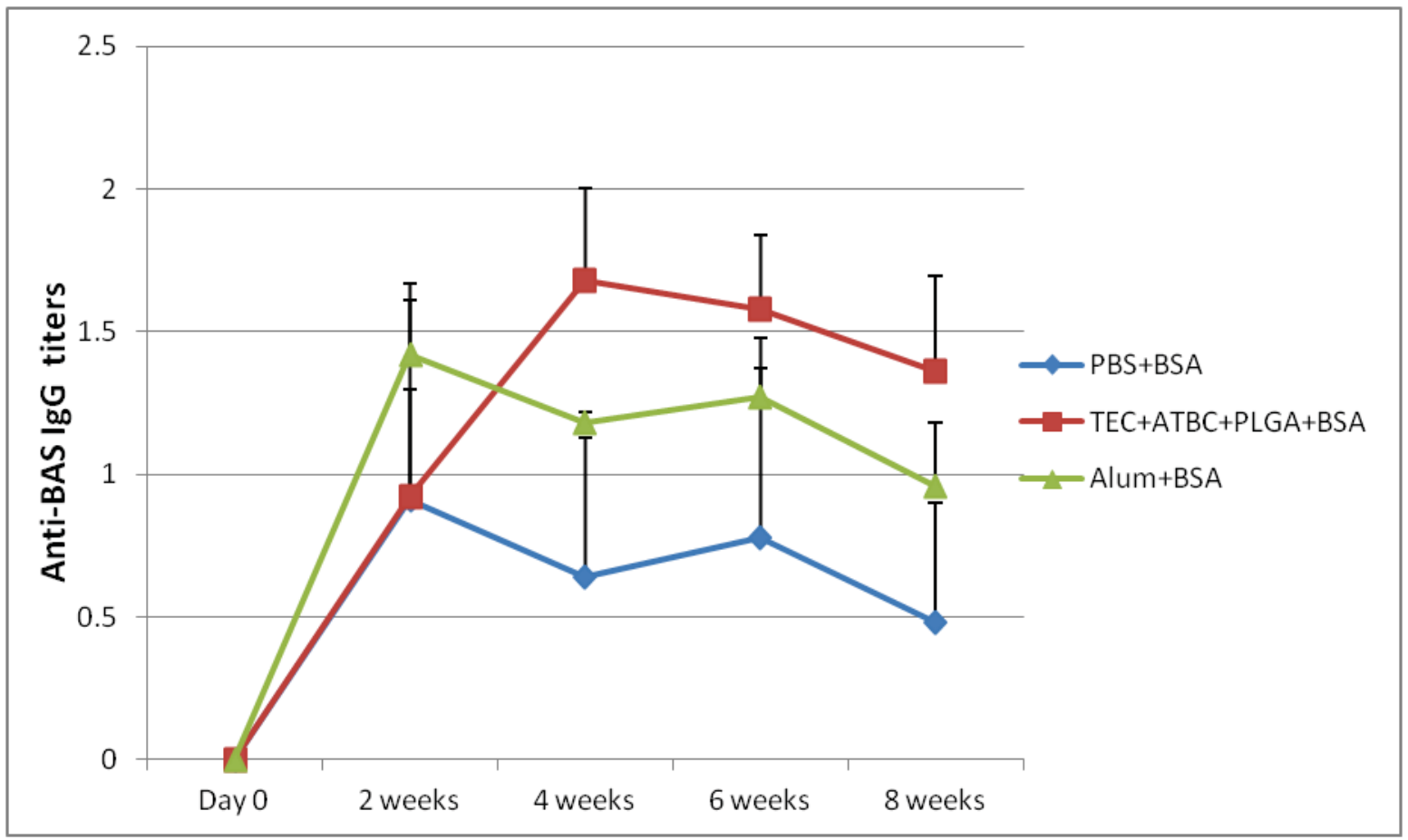

Figure 2-7. Rabbit serum anti-BSA IgG titers with multiple time points. 


\section{CHAPTER 3. DEVELOPMENT OF NEW VACCINE FORMULATIONS WITH DELAYED IMMUNE RESPONSE BASED ON ADJUGEL SYSTEM USING ORGANIC SOLVENT NMP}

\subsection{Introduction}

From the three experiments in Chapter 2, we could draw the conclusion that the AdjuGel system is a new type of vaccine adjuvant or delivery system, which has significant potential for sustained release activity and development of single dose vaccines.

The Oil-based formulations are one of the most classic formulations in vaccine adjuvants. Previous studies showed neither oils nor surfactants alone can induce strong immune responses.[5] we compared the oil- based vaccine adjuvants to the AdjuGel system and found the AdjuGel system displayed a similar mode of action to oil-based vaccine adjuvants. In AdjuGel systems, ATBC could be considered the oil and polymer PLGA could be considered the surfactant. Just like oil-based adjuvants, removal of either ATBC or Polymer PLGA could lead to the loss of strong immune response. However, the hydrophobic polymer PLGA is not a surfactant. Under normal conditions, PLGA will not act as a surfactant because of the high molecular weight. When exposed to aqueous media such as PBS buffers or tissues, hydrophobic polymer PLGA or PLA will absorb water and ester linkages will break by hydrolysis, which leads long polymer chains to break into shorter ones. As a result, the reduction in molecular weight produces an increase in hydrophilicity. When the hydrophilicity lipophilicity balance (HLB) of polymer reaches a suitable scale, hydrophobic polymer PLGA or PLA will show the characteristics of surfactants, which in turn may alter the AdjuGel system to be more similar to classic oil-based vaccine adjuvant and induce immune responses.

Based on the hypothesis above, AdjuGel system should exhibit a delayed immune response because degradation of PLGA polymer will need some time in vivo. However, in the rabbit experiment, we did not see a delayed response. The reason is probably because of the intramuscular administration route. Injected into muscular tissues, the ISI system will be degraded very fast. Limited space in muscle prevents ISI system from forming a single implant with integrity. Also plenty of blood circulation in muscular tissues causes a high rate of metabolism, which also accelerates the degradation of PLGA polymer. So there is no delayed response demonstrated in the rabbit experiment. In order to slow the degradation rate, we chose to use a subcutaneous injection instead of intramuscular injection.

For the new animal experiment, two different PLGA polymers were chosen to test if they could provide delayed immune responses. The first one is a relatively hydrophilic and fast degradation polymer; the second PLGA polymer is a more hydrophobic and slow degradation polymer. We predicted that the formulation with second PLGA polymer could provide a delay immune response compared to the formulation with first PLGA polymer. 
Because AdjuGel system is a new formulation for vaccine adjuvant, type of immunity (humoral or cell mediate immunity) need to be determined. It's well known that Alum will initiate humoral immune response (Th2 response) rather than cell mediate immune response (Th1 response). [52] We used Alum as control and measured total IgG titers, isotype IgG1 titers (represent humoral immunity) and isotype IgG2a titers (represent cell mediate immunity) in the new experiment.

\subsection{Experimental Procedures}

\subsubsection{Materials}

35 BABL/c female mice (6-8 weeks) were ordered from Jackson Lab. The biodegradable polymer PLGA was ordered from Lactel Absorbable Polymers, Durect Corporation, Pelham, AL, USA. NMP was obtained from ISP Pharm Technologies; Acetyl Tributyl Citrate (ATBC) was obtained from Morflex Inc, Greensboro, NC, USA; Lyophilized Ovalbumin (OVA) as the antigen was purchased from Sigma; Alum was ordered from Thermo Scientific.

\subsubsection{Methods}

Formulation process of the vaccines (all steps were prepared aseptically): 1 . Prepare four groups of AdjuGels consisting of a blend of PLGA and a combination of NMP and ATBC at $50^{\circ} \mathrm{C}-55^{\circ} \mathrm{C}$. 2. An appropriate quantity of gel was weighed and transferred in to an autoclaved clean glass vial. An appropriate quantity of OVA was weighed and blended with the gel and resulting mixture was stirred to obtain uniform mixture of OVA-loaded gel. 3. Each dose of OVA-loaded gel contains 50ug of OVA per $0.1 \mathrm{ml}$ gel. The final formulation is a suspension of OVA in the clear gel solution.

\subsubsection{In vitro studies}

One mililiter of each vaccine gel (formulations are same with group 4, 5, 6 and 7 below) was loaded into a $20 \mathrm{ml}$ autoclaved clean bottle, and then $4.0 \mathrm{ml} \mathrm{PBS} \mathrm{(pH} \mathrm{7.4,}$ $0.01 \mathrm{M}$ ) was added. The sample bottles were kept in $37^{\circ} \mathrm{C}$ shaker at $60 \mathrm{rpm}$. At $24 \mathrm{hrs}$ and then at two days and one week intervals, $3.0 \mathrm{ml}$ PBS sample was collected and analyzed for ovalbumin and ATBC concentration with Enzyme immunoassay (EIA) and high performance liquid chromatograph (HPLC) method. $3.0 \mathrm{ml}$ fresh PBS was added after every collection. Release profiles were calculated by cumulative release with incubation time.

\subsection{Analytical method development for OVA (EIA)}

96-well microtiter plates (high protein binding plates from Costar) were coated overnight with $100 \mu \mathrm{l}$ of ovalbumin standard solutions and samples for test at $4{ }^{\circ} \mathrm{C}$. To 
remove unbound OVA, plates were washed three times with PBS (pH 7.4) containing $0.05 \%$ Tween 20 (PBST). Primary antibody from rabbit were diluted in PBST and loaded into the plates with $100 \mu \mathrm{L} /$ well. The plates were then incubated for two hours at room temperature. The plates were again washed three times with PBST followed by addition of $100 \mu \mathrm{L}$ of PBST containing Horseradish Peroxidase (HRP) -conjugated goat antirabbit IgG (diluted 1:4000) (Abcam, ab6721). After a two hour incubation period at room temperature, the plates were washed three times with PBST followed by the addition of $200 \mu \mathrm{L}$ of $0.4 \mathrm{mg} / \mathrm{ml}$ OPD Peroxidase Substrate (Sigma P9187, Sigma-Aldrich, St. Louis, MO) and allowed to react for 30 minutes at room temperature. The optical density (OD) of the reaction was measured at $450 \mathrm{~nm}$ using a Plate Reader. The sample OVA concentrations were calculated in accordance to standard curve.

\subsubsection{2. $\quad$ Analytical method development for ATBC (HPLC)}

ATBC was analyzed by reverse phase (RP)-HPLC using a C18 column, $150 \mathrm{~mm}$ $\times 4.60 \mathrm{~mm}$ (SGE Analytical Science. Part No. 250112). The mobile phase was a 7:3 mixture of acetonitrile (HPLC grade) and water (HPLC grade). The flow rate was set at $1 \mathrm{ml} / \mathrm{min}$ and the injector volume was $50 \mu \mathrm{m}$. UV absorbance was measured at $234 \mathrm{~nm}$ using a photodiode array detector equipped with the high performance liquid chromatography system (HPLC). The results were calculated based on the readings from a standard series of ATBC. [53]

\subsubsection{Immunization of animals}

For all the immunization experiments, Lyophilized OVA protein was dissolved in PBS buffer or was suspended in different AdjuGel systems with organic solvent NMP immediately before injection to obtain a concentration of $500 \mathrm{ug} / \mathrm{ml}$. All mice were given single S.C. inoculation in lower back at day 0. Experimental assignment was shown in Table 3-1.

\subsubsection{Procedures}

All groups were inoculated S. C. once at day 0 and blood samples were collected every 2 weeks in the first 2 months and every 3 or 4 weeks thereafter. Blood samples were serially diluted with $0.05 \%$ Tween 20 in PBS: 1:100, 1:1,000, 1:10,000 and 1:100,000. IgG titers were checked by ELISA (Enzyme-Linked Immunosorbant Assay); use Log10EC50 as a measure of immune response. EC50 means half maximal effective concentration and the absorbance of 1:100 dilution was used as the maximum.

\subsubsection{Enzyme linked immunosorbent assay (ELISA)}

96-well microtiter plates (high protein binding plates from Costar) were coated overnight with $100 \mu \mathrm{l}$ coating solution containing $3 \mu \mathrm{g} / \mathrm{mL}$ ovalbumin (OVA) at $4{ }^{\circ} \mathrm{C}$. To remove unbound OVA, plates were washed three times with PBS ( $\mathrm{pH} 7.4)$ containing $0.05 \%$ Tween 20 (PBST). Serum samples (100 $\mu \mathrm{L} /$ well) from individual mice were 
Table 3-1. Mouse experimental group assignment in OVA study.

\begin{tabular}{|c|c|c|c|}
\hline Group & Treatment & $\begin{array}{l}\text { Number } \\
\text { of mouse }\end{array}$ & Description \\
\hline 1 & Control & 5 & S.C. injection with PBS buffer and OVA \\
\hline 2 & $\begin{array}{l}\text { ATBC emulsion } \\
+ \text { OVA }\end{array}$ & 5 & S.C. injection ATBC emulsion and OVA \\
\hline 3 & ALUM+OVA & 5 & S.C. injection with ALUM and OVA \\
\hline 4 & AdjuGel 1 + OVA & 5 & S.C. injection with AdjuGel 1 and OVA \\
\hline 5 & AdjuGel $2+\mathrm{OVA}$ & 5 & S.C. injection with AdjuGel 2 and OVA \\
\hline 6 & AdjuGel $3+$ OVA & 5 & S.C. injection with AdjuGel 3 and OVA \\
\hline 7 & AdjuGel $4+$ OVA & 5 & S.C. injection with AdjuGel 4 and OVA \\
\hline $\begin{array}{l}\text { Ovalbu } \\
\text { AdjuG } \\
\text { concen } \\
\text { AdjuG } \\
\text { concen } \\
\text { AdjuG } \\
\text { ATBC } \\
\text { AdjuG } \\
\text { ATBC } \\
\text { The PL } \\
\text { (w/w). }\end{array}$ & $\begin{array}{l}\text { nin is } 50 \text { ug per inject } \\
1 \text { 1: NMP, ATBC an } \\
\text { ation is } 30 \%(\mathrm{w} / \mathrm{w}) \text {. } \\
\text { 2: NMP, ATBC an } \\
\text { ation is } 15 \%(\mathrm{w} / \mathrm{w}) \text {. } \\
13: \mathrm{NMP}, \mathrm{ATBC} \text { an } \\
\text { oncentration is } 30 \% \\
14: \mathrm{NMP}, \mathrm{ATBC} \text { an } \\
\text { oncentration is } 15 \% \\
\text { JA concentration in }\end{array}$ & $\begin{array}{l}\text { on. The vol } \\
\text { low molec } \\
\text { low molec } \\
\text { high molec } \\
\text { w/w). } \\
\text { high molec } \\
\text { N/w). } \\
\text { djuGel } 1, \text { A }\end{array}$ & $\begin{array}{l}\text { Ime is 100ul per injection. } \\
\text { lar weight PLGA (50:50; IV: 0.65). The ATBC } \\
\text { lar weight PLGA (50:50; IV: 0.65). The ATBC } \\
\text { ular weight PLGA (85:15; IV: 0.65). The } \\
\text { ular weight PLGA (85:15; IV: } 0.65) \text {. The } \\
\text { djuGel 2, AdjuGel } 3 \text { and AdjuGel } 4 \text { is } 20 \%\end{array}$ \\
\hline
\end{tabular}


serially diluted in PBST: 1:100, 1:1,000, 1:10,000 and 1:100,000. The plates were then incubated for two hours at room temperature. The plates were again washed three times with PBST followed by addition of $100 \mu \mathrm{L}$ of PBST containing Horseradish Peroxidase (HRP) -conjugated Goat Anti-Mouse IgG (diluted 1:4000) (Abcam, ab6789), or Horseradish Peroxidase (HRP) -conjugated Goat Anti-Mouse IgG1 (diluted 1:4000) (Abcam, ab97240) or Horseradish Peroxidase (HRP) -conjugated Goat Anti-Mouse IgG2a (Abcam, ab97241). After a two hour incubation period at room temperature, the plates were washed three times with PBST followed by the addition of $200 \mu \mathrm{L}$ of 0.4 $\mathrm{mg} / \mathrm{ml}$ OPD Peroxidase Substrate (Sigma P9187, Sigma-Aldrich, St. Louis, MO) and allowed to react for 30 minutes at room temperature. The optical density (OD) of the reaction was measured at $450 \mathrm{~nm}$ using a Plate Reader. Software BioDataFit was applied to deal with the data by the four-parameter model. Serum titers are reported with $\log 10 \mathrm{EC} 50$.

\subsubsection{Statistical analysis}

Results are expressed as mean \pm S.D. Statistical analysis was carried out for analyses of differences between means of serum antibody titers using Student's twotailed $t$-test. Differences in means were accepted as significant if $P$ was less than 0.05 .

\subsection{Results}

\subsubsection{In vitro release- Ovalbumin}

The study of OVA In vitro release showed that the four AdjuGel groups could provide continuous sustained release of OVA (Table 3-2 and Figure 3-1). In the groups of low molecular weight PLGA, OVA could be released up to seven weeks; in the groups of high molecular weight PLGA, OVA could be released up to thirteen weeks. The high concentration of ATBC could decrease initial burst release of OVA because ATBC increased hydrophobicity in the AdjuGel groups, which in turn decrease OVA initial burst release. However, the high concentration of ATBC didn't prolong the whole release period. There are burst releases in all AdjuGel groups. On day 3, 10\% of total ovalbumin was released in group AdjuGel 1; $24 \%$ of total ovalbumin was released in group AdjuGel $2 ; 11 \%$ of total ovalbumin was released in group AdjuGel 3;14\% of total ovalbumin was released in group AdjuGel 4.

\subsubsection{In vitro release- ATBC}

The study of ATBC in vitro release showed that the four AdjuGel groups could provide delayed release of ATBC (Table 3-3 and Figure 3-2). In the groups of low molecular weight PLGA, ATBC wouldn't be released significantly until the fifth weeks; in the groups of high molecular weight PLGA, ATBC wouldn't be released significantly until the tenth weeks. 
Table 3-2. Data of in vitro OVA accumulative release with four AdjuGel groups.

\begin{tabular}{lcccc}
\hline Time & AdjuGel 1 & AdjuGel 2 & AdjuGel 3 & AdjuGel 4 \\
\hline Day 0 & $0 \pm 0$ & $0 \pm 0$ & $0 \pm 0$ & $0 \pm 0$ \\
Day 1 & $0.06 \pm 0.03$ & $0.12 \pm 0.02$ & $0.05 \pm 0.03$ & $0.07 \pm 0.01$ \\
Day 3 & $0.1 \pm 0.02$ & $0.24 \pm 0.06$ & $0.11 \pm 0.02$ & $0.14 \pm 0.01$ \\
Week 1 & $0.13 \pm 0.01$ & $0.42 \pm 0.17$ & $0.18 \pm 0.03$ & $0.33 \pm 0.06$ \\
Week 2 & $0.16 \pm 0.02$ & $0.57 \pm 0.07$ & $0.27 \pm 0.04$ & $0.44 \pm 0.04$ \\
Week 3 & $0.33 \pm 0.03$ & $0.73 \pm 0.05$ & $0.44 \pm 0.16$ & $0.57 \pm 0.05$ \\
Week 4 & $0.75 \pm 0.08$ & $0.87 \pm 0.05$ & $0.58 \pm 0.16$ & $0.64 \pm 0.03$ \\
Week 5 & $0.9 \pm 0.02$ & $0.95 \pm 0.03$ & $0.63 \pm 0.02$ & $0.68 \pm 0.01$ \\
Week 6 & $0.95 \pm 0$ & $0.98 \pm 0.01$ & $0.67 \pm 0.02$ & $0.73 \pm 0.01$ \\
Week 7 & $1 \pm 0.01$ & $1 \pm 0.01$ & $0.75 \pm 0.01$ & $0.81 \pm 0.01$ \\
Week 8 & & & $0.83 \pm 0.01$ & $0.88 \pm 0.02$ \\
Week 9 & & & $0.88 \pm 0$ & $0.93 \pm 0.01$ \\
Week 10 & & & $0.92 \pm 0$ & $0.96 \pm 0.01$ \\
Week 11 & & & $0.96 \pm 0$ & $0.98 \pm 0.01$ \\
Week 12 & & & $0.98 \pm 0$ & $0.99 \pm 0$ \\
Week 13 & & & $1 \pm 0$ & $1 \pm 0$ \\
\hline AdjuCe1 1: NMP, ATBC an
\end{tabular}

AdjuGel 1: NMP, ATBC and low molecular weight PLGA (50:50; IV: 0.65). The ATBC concentration is $30 \%(\mathrm{w} / \mathrm{w})$; AdjuGel 2: NMP, ATBC and low molecular weight PLGA (50:50; IV: 0.65). The ATBC concentration is 15\% (w/w); AdjuGel 3: NMP, ATBC and high molecular weight PLGA (85:15; IV: 0.65$)$. The ATBC concentration is $30 \%(\mathrm{w} / \mathrm{w})$; AdjuGel 4: NMP, ATBC and high molecular weight PLGA (85:15; IV: 0.65). The ATBC concentration is $15 \%(\mathrm{w} / \mathrm{w})$. Data were presented as Mean $\pm \mathrm{S}$. D. 


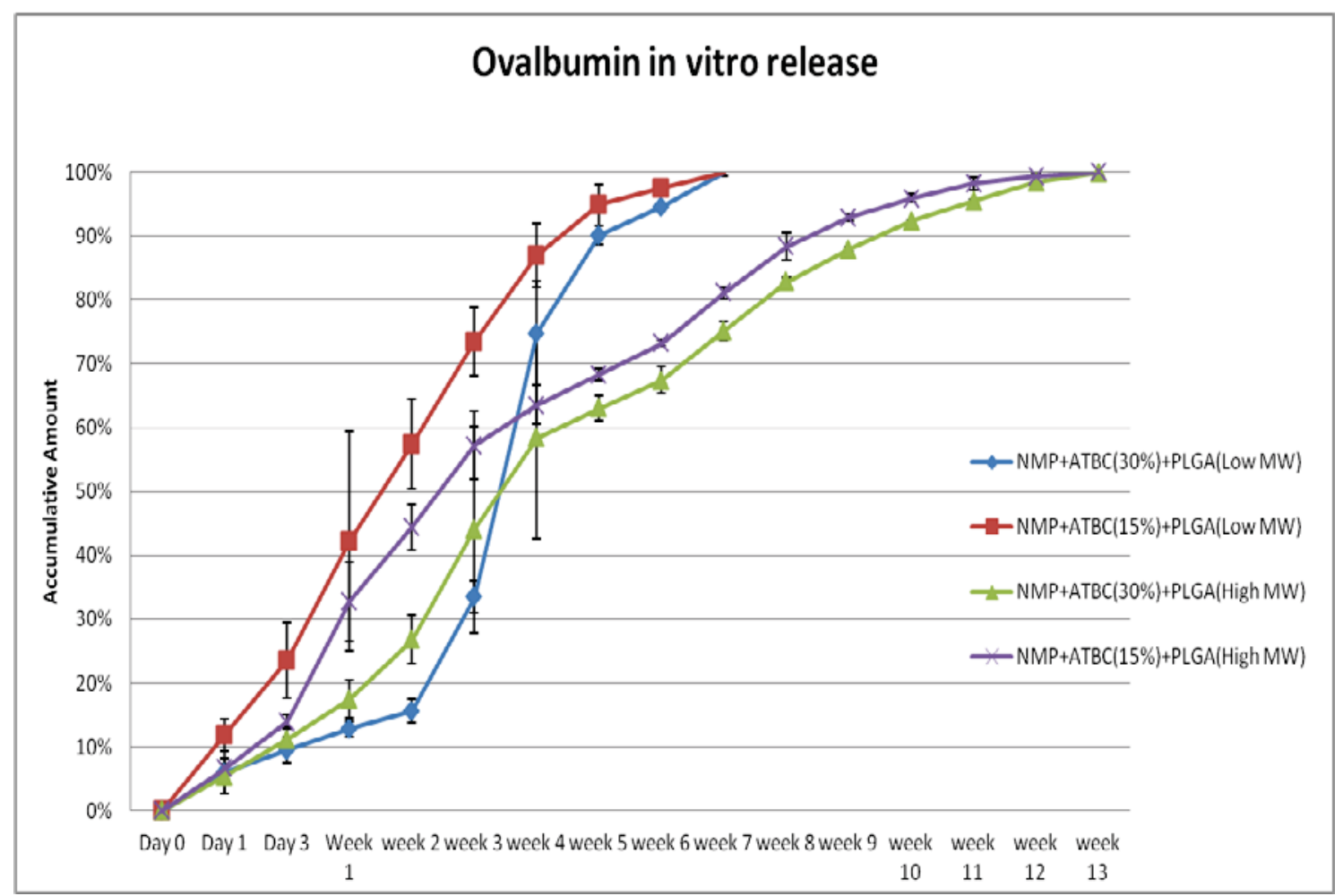

Figure 3-1. In vitro OVA release study with four AdjuGel groups.

AdjuGel 1: NMP, ATBC and low molecular weight PLGA (50:50; IV: 0.65). The ATBC concentration is 30\% (w/w); AdjuGel 2: NMP, ATBC and low molecular weight PLGA (50:50; IV: 0.65). The ATBC concentration is 15\% (w/w); AdjuGel 3: NMP, ATBC and high molecular weight PLGA (85:15; IV: 0.65). The ATBC concentration is $30 \%(\mathrm{w} / \mathrm{w})$; AdjuGel 4: NMP, ATBC and high molecular weight PLGA (85:15; IV: 0.65). The ATBC concentration is $15 \%(\mathrm{w} / \mathrm{w})$. 
Table 3-3. Data of in vitro ATBC accumulative release with four AdjuGel groups.

\begin{tabular}{|c|c|c|c|c|}
\hline Time & AdjuGel 1 & AdjuGel 2 & AdjuGel 3 & AdjuGel 4 \\
\hline Day 0 & $0 \pm 0$ & $0 \pm 0$ & $0 \pm 0$ & $0 \pm 0$ \\
\hline Day 1 & $0.0004 \pm 0.0007$ & $0.0004 \pm 0.0001$ & $0.0002 \pm 0.0001$ & $0.0004 \pm 0.0001$ \\
\hline Day 3 & $0.0007 \pm 0$ & $0.0007 \pm 0$ & $0.0005 \pm 0$ & $0.0008 \pm 0.0001$ \\
\hline Week 1 & $0.001 \pm 0$ & $0.0011 \pm 0$ & $0.0007 \pm 0$ & $0.0012 \pm 0.0003$ \\
\hline Week 2 & $0.0013 \pm 0$ & $0.0014 \pm 0$ & $0.0009 \pm 0$ & $0.0015 \pm 0$ \\
\hline Week 3 & $0.0015 \pm 0$ & $0.0018 \pm 0.0001$ & $0.0011 \pm 0$ & $0.0019 \pm 0.0002$ \\
\hline Week 4 & $0.0018 \pm 0$ & $0.0022 \pm 0.0002$ & $0.0014 \pm 0$ & $0.0023 \pm 0.0001$ \\
\hline Week 5 & $0.2506 \pm 0.3838$ & $0.1341 \pm 0.3741$ & $0.0023 \pm 0.0005$ & $0.0028 \pm 0.0007$ \\
\hline Week 6 & $0.5585 \pm 0.3067$ & $0.3531 \pm 0.2777$ & $0.0033 \pm 0.0012$ & $0.0031 \pm 0$ \\
\hline Week 7 & $1 \pm 0.3087$ & $1 \pm 0.3476$ & $0.0038 \pm 0.0002$ & $0.0038 \pm 0.0017$ \\
\hline Week 8 & & & $0.0222 \pm 0.1081$ & $0.0069 \pm 0.0454$ \\
\hline Week 9 & & & $0.0246 \pm 0.0043$ & $0.0094 \pm 0.0026$ \\
\hline Week 10 & & & $0.0945 \pm 0.258$ & $0.0234 \pm 0.1503$ \\
\hline Week 11 & & & $0.1079 \pm 0.0355$ & $0.0308 \pm 0.0532$ \\
\hline Week 12 & & & $0.2857 \pm 0.3498$ & $0.1137 \pm 0.425$ \\
\hline Week 13 & & & $1 \pm 0.2423$ & $0.9998 \pm 0.3204$ \\
\hline \multicolumn{5}{|c|}{$\begin{array}{l}\text { AdjuGel 1: NMP, ATBC and low molecular weight PLGA (50:50; IV: 0.65). The ATBC } \\
\text { concentration is } 30 \%(\mathrm{w} / \mathrm{w}) \text {; AdjuGel 2: NMP, ATBC and low molecular weight PLGA } \\
(50: 50 ; \text { IV: } 0.65) \text {. The ATBC concentration is } 15 \%(\mathrm{w} / \mathrm{w}) \text {; AdjuGel 3: NMP, ATBC and } \\
\text { high molecular weight PLGA ( } 85: 15 ; \text { IV: } 0.65) \text {. The ATBC concentration is } 30 \%(\mathrm{w} / \mathrm{w}) \text {; } \\
\text { AdjuGel 4: NMP, ATBC and high molecular weight PLGA }(85: 15 ; \mathrm{IV}: 0.65) \text {. The } \\
\text { ATBC concentration is } 15 \%(\mathrm{w} / \mathrm{w}) \text {. Data were presented as Mean } \pm \mathrm{S} \text {. D. }\end{array}$} \\
\hline
\end{tabular}




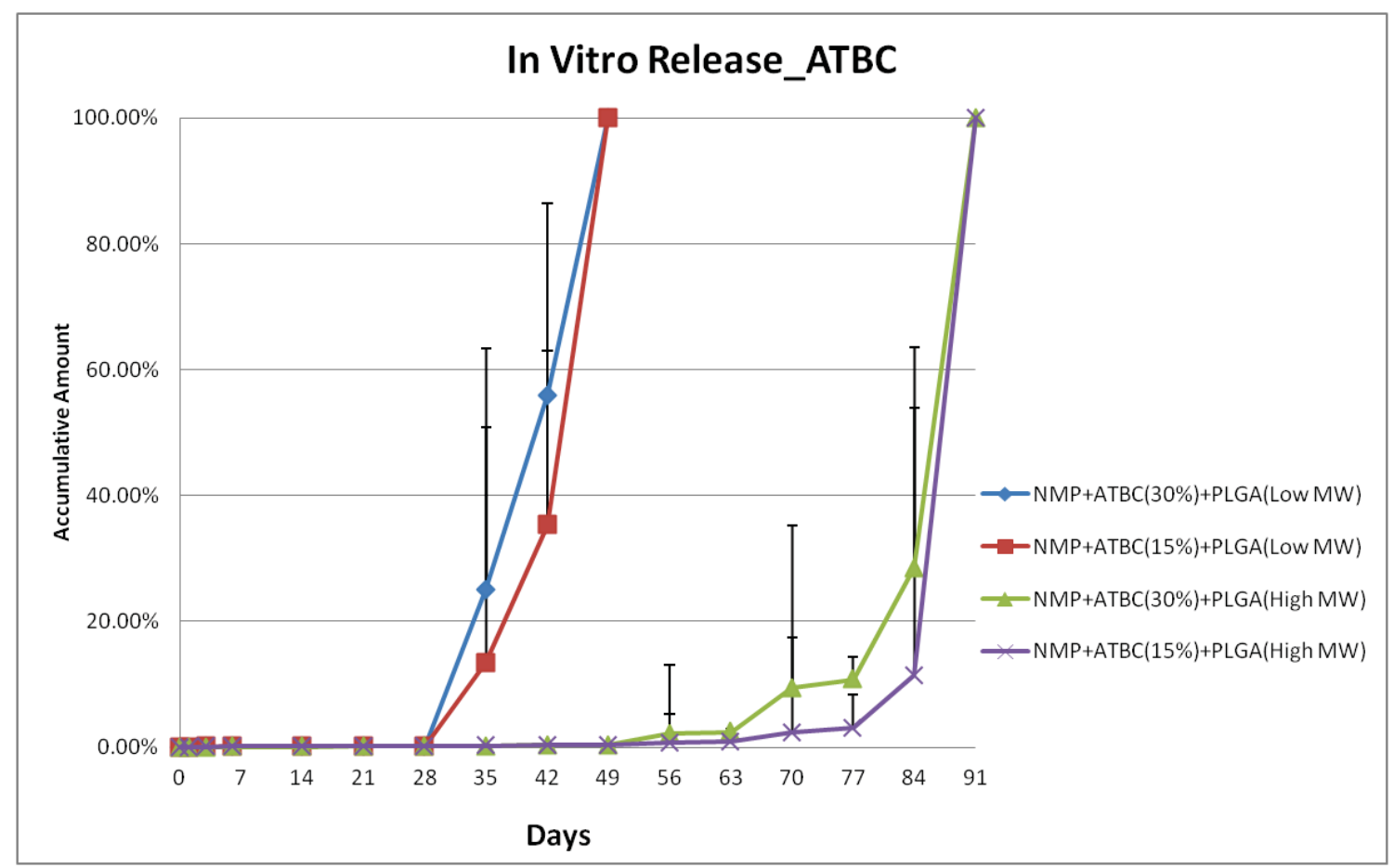

Figure 3-2. In vitro ATBC release study with four AdjuGel groups.

AdjuGel 1: NMP, ATBC and low molecular weight PLGA (50:50; IV: 0.65). The ATBC concentration is 30\% (w/w); AdjuGel 2: NMP, ATBC and low molecular weight PLGA (50:50; IV: 0.65). The ATBC concentration is 15\% (w/w); AdjuGel 3: NMP, ATBC and high molecular weight PLGA (85:15; IV: 0.65). The ATBC concentration is $30 \%(\mathrm{w} / \mathrm{w})$; AdjuGel 4: NMP, ATBC and high molecular weight PLGA (85:15; IV: 0.65). The ATBC concentration is $15 \%(\mathrm{w} / \mathrm{w})$. 


\subsubsection{In vivo studies}

$48 \mathrm{hrs}$ after S.C. injection, all mice in group 4, 5, 6 and 7 showed mild to moderate skin lesions (local inflammatory reaction at the injection sites). The reason is not very clear although it is probably related to NMP. The skin lesions were recovered within two weeks after treatment with antibiotic ointment.

The IgG titers in all AdjuGel groups showed significantly greater than those in the negative control (group 1) between week 4 and week 8 ( $\mathrm{P}<0.05$, Table 3-4 and Figure 3-3). But there was no significant difference between AdjuGel groups and the positive controls (group 2 and 3 ) between week 4 and week 8 ( $P>0.05$ ). Also the AdjuGel groups didn't show any delayed immune responses, but showed normal immune responses profiles similar to positive controls.

The IgG1 titers in all AdjuGel groups showed significantly greater than those in the negative control (group 1) between week 4 and week $8(\mathrm{P}<0.05$, Table 3-5 and Figure 3-4). However, the AdjuGel groups didn't show any delayed immune responses, but showed normal immune responses profiles similar to positive controls.

The IgG2a titers of all groups were very low and no significant difference among them from week 4 to week 8 (Table 3-6 and Figure 3-5).

\subsection{Discussion}

Even though a delayed ATBC release was presented in in vitro study, the in vivo mouse studies clearly showed the immune response was detected in two weeks, which means that there was probably no delayed ATBC release in vivo.

Why is there a difference between the in vitro and in vivo results?

From the local inflammation in the injection sites and surrounding skin lesions, we believed the organic solvent N-Methyl-2-pyrrolidone (NMP) is the key component which induced the inflammation and may accelerate the degradation of PLGA polymer, which in turn could cause the fast immune responses right after the injections.

NMP was used in some FDA approved drugs, such as Eligard ${ }^{\circledR}$ (leuprolide acetate for in situ implant depot). In 1998 a study with rhesus monkeys showed that NMP is a safe organic solvent in a drug delivery system. [54] Our previous rabbit experiment also did not show any side effects with the injection of gel formulations containing NMP. Although NMP seemed safe in the experiments of monkey and rabbit, it displayed pretty strong tissue irritation and caused local inflammation at the injection sites in our mouse study. In 2001, Kranz and his colleagues studied three biocompatible organic solvents: NMP, DMSO and 2-pyrrolidone for the acute toxicity or tissue damage following intramuscular injection with rats.[50] They found the rank order of tissue damage of the solvents was 2-pyrrolidone $<$ DMSO $<$ NMP. (See Figure 3-6 below)[50] 
Table 3-4. The results of serum anti-OVA IgG antibody titers for group 1, 2, 3, 4, 5, 6 and 7 at different time points of day 0 , week 2, 4, 6, 8, 11, 14 and 18 .

\begin{tabular}{lccccccc}
\hline Time & G1 & G2 & G3 & G4 & G5 & G6 & G7 \\
\hline Day 0 & $0 \pm 0$ & $0 \pm 0$ & $0 \pm 0$ & $0 \pm 0$ & $0 \pm 0$ & $0 \pm 0$ & $0 \pm 0$ \\
2 weeks & $0.18 \pm 0.05$ & $0.81 \pm 0.14$ & $0.56 \pm 0.21$ & $0.83 \pm 0.24$ & $0.43 \pm 0.31$ & $1.01 \pm 0.51$ & $0.66 \pm 0.35$ \\
4 weeks & $0.28 \pm 0.15$ & $0.92 \pm 0.14$ & $0.94 \pm 0.25$ & $0.91 \pm 0.43$ & $0.59 \pm 0.5$ & $1.14 \pm 0.5$ & $0.79 \pm 0.47$ \\
6 weeks & $0.35 \pm 0.13$ & $0.88 \pm 0.2$ & $1.03 \pm 0.19$ & $0.73 \pm 0.78$ & $1.23 \pm 0.5$ & $1.24 \pm 0.76$ & $0.84 \pm 0.49$ \\
8 weeks & $0.4 \pm 0.27$ & $0.99 \pm 0.16$ & $1.18 \pm 0.5$ & $1 \pm 0.69$ & $0.88 \pm 1.09$ & $1.32 \pm 0.79$ & $0.88 \pm 0.67$ \\
11 weeks & $0.34 \pm 0.29$ & $0.98 \pm 0.23$ & $1.19 \pm 0.22$ & $0.72 \pm 0.5$ & & $1.26 \pm 0.74$ \\
14 weeks & $0.63 \pm 0.31$ & $1.03 \pm 0.31$ & $1.14 \pm 0.3$ & $0.71 \pm 0.54$ & & $1.13 \pm 0.73$ & $0.91 \pm 0.67$ \\
18 weeks & $0.2 \pm 0$ & $0.38 \pm 0.1$ & $0.38 \pm 0.1$ & $0.4 \pm 0.2$ & & $0.58 \pm 0.25$ & $0.6 \pm 0.3$ \\
P value & & & & $0.693 *$ & $0.347 * *$ & $0.422^{* * *}$ & $0.884 * * *$ \\
\hline
\end{tabular}

Serum anti-OVA total IgG titers were reported with Log10EC50 and data were presented as Mean \pm S. D.

P values with 6 weeks results (*G4 vs. G2; **G5 vs. G2; ***G6 vs. G2;****G7 vs. G2) 


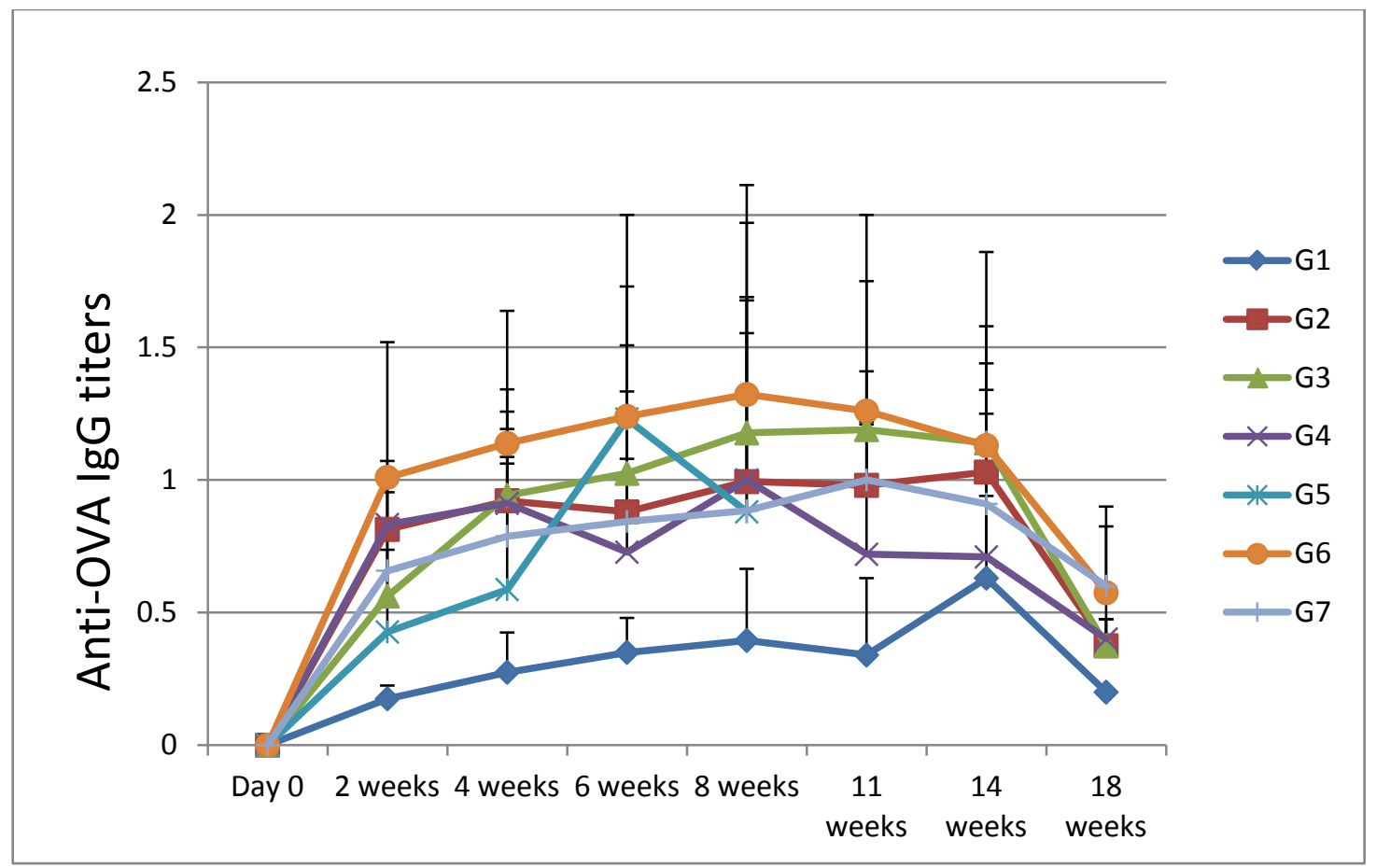

Figure 3-3. Mice serum anti-OVA IgG antibody titers.

G1, S.C. injection with PBS buffer and OVA; G2, S.C. injection ATBC emulsion and OVA; G3, S.C. injection with ALUM and OVA; G4, S.C. injection with AdjuGel 1 and OVA; G5, S.C. injection with AdjuGel 2 and OVA; G6, S.C. injection with AdjuGel 3 and OVA; G7, S.C. injection with AdjuGel 4 and OVA. 
Table 3-5. The results of serum anti-OVA IgG1 antibody titers for group 1, 2, 3, 4, 5, 6 and 7 at different time points of day 0 , week $2,4,6,8,11,14$ and 18 .

\begin{tabular}{lccccccc}
\hline Time & G1 & G2 & G3 & G4 & G5 & G6 & G7 \\
\hline Day 0 & $0 \pm 0$ & $0 \pm 0$ & $0 \pm 0$ & $0 \pm 0$ & $0 \pm 0$ & $0 \pm 0$ & $0 \pm 0$ \\
2 weeks & $0.15 \pm 0.06$ & $0.82 \pm 0.26$ & $0.69 \pm 0.28$ & $0.77 \pm 0.38$ & $0.37 \pm 0.29$ & $1.17 \pm 0.54$ & $0.7 \pm 0.38$ \\
4 weeks & $0.23 \pm 0.12$ & $0.98 \pm 0.09$ & $0.96 \pm 0.31$ & $1.02 \pm 0.62$ & $0.61 \pm 0.63$ & $1.26 \pm 0.49$ & $0.85 \pm 0.63$ \\
6 weeks & $0.38 \pm 0.17$ & $0.95 \pm 0.21$ & $1.16 \pm 0.25$ & $0.73 \pm 0.8$ & $1.75 \pm 0.13$ & $1.38 \pm 0.81$ & $0.87 \pm 0.56$ \\
8 weeks & $0.41 \pm 0.31$ & $1.03 \pm 0.26$ & $1.26 \pm 0.47$ & $1.06 \pm 0.75$ & $0.91 \pm 1.16$ & $1.43 \pm 0.82$ & $0.93 \pm 0.72$ \\
11 weeks & $0.32 \pm 0.31$ & $1.06 \pm 0.2$ & $1.34 \pm 0.23$ & $0.79 \pm 0.62$ & & $1.3 \pm 0.84$ & $1.02 \pm 0.81$ \\
14 weeks & $0.68 \pm 0.37$ & $1.14 \pm 0.34$ & $1.26 \pm 0.34$ & $0.78 \pm 0.73$ & & $1.22 \pm 0.75$ & $0.98 \pm 0.76$ \\
18 weeks & $0.1 \pm 0$ & $0.3 \pm 0.1$ & $0.3 \pm 0.1$ & $0.4 \pm 0.2$ & & $0.45 \pm 0.25$ & $0.55 \pm 0.35$ \\
\hline
\end{tabular}

Serum anti-OVA IgG1 titers were reported with Log10EC50 and data were presented as Mean \pm S. D. 


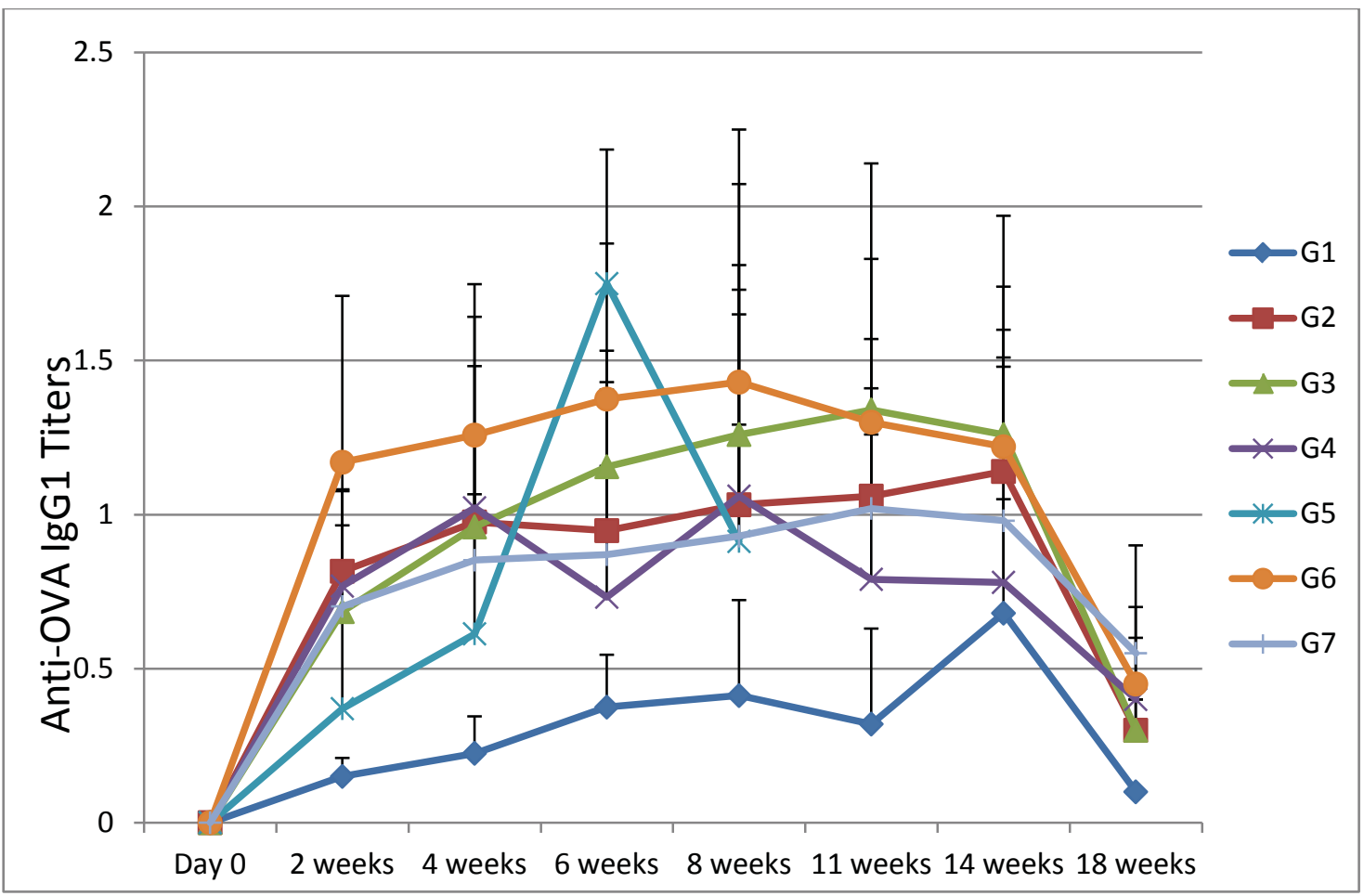

Figure 3-4. Mice serum anti-OVA IgG1 antibody titers.

G1, S.C. injection with PBS buffer and OVA; G2, S.C. injection ATBC emulsion and OVA; G3, S.C. injection with ALUM and OVA; G4, S.C. injection with AdjuGel 1 and OVA; G5, S.C. injection with AdjuGel 2 and OVA; G6, S.C. injection with AdjuGel 3 and OVA; G7, S.C. injection with AdjuGel 4 and OVA. 
Table 3-6. The results of serum anti-OVA IgG2a antibody titers for group 1, 2, 3, 4, 5, 6 and 7 at different time points of day 0 , week 2, 4, 6 and 8 .

\begin{tabular}{lccccccc}
\hline Time & G1 & G2 & G3 & G4 & G5 & G6 & G7 \\
\hline Day 0 & $0 \pm 0$ & $0 \pm 0$ & $0 \pm 0$ & $0 \pm 0$ & $0 \pm 0$ & $0 \pm 0$ & $0 \pm 0$ \\
2 weeks & $0.06 \pm 0.005$ & $0.06 \pm 0.008$ & $0.07 \pm 0.029$ & $0.17 \pm 0.12$ & $0.06 \pm 0.006$ & $0.06 \pm 0$ & $0.06 \pm 0.008$ \\
4 weeks & $0.06 \pm 0.005$ & $0.07 \pm 0.009$ & $0.07 \pm 0.013$ & $0.06 \pm 0.011$ & $0.06 \pm 0.006$ & $0.08 \pm 0.012$ & $0.07 \pm 0.008$ \\
6 weeks & $0.06 \pm 0.005$ & $0.09 \pm 0.025$ & $0.1 \pm 0.021$ & $0.07 \pm 0.005$ & $0.1 \pm 0.01$ & $0.1 \pm 0.007$ & $0.07 \pm 0.019$ \\
8 weeks & $0.06 \pm 0.006$ & $0.1 \pm 0.026$ & $0.1 \pm 0.046$ & $0.1 \pm 0.035$ & $0.08 \pm 0.034$ & $0.12 \pm 0.065$ & $0.11 \pm 0.068$ \\
\hline
\end{tabular}

Serum anti-OVA IgG2a titers were reported with Log10EC50 and data were presented as Mean \pm S. D. 


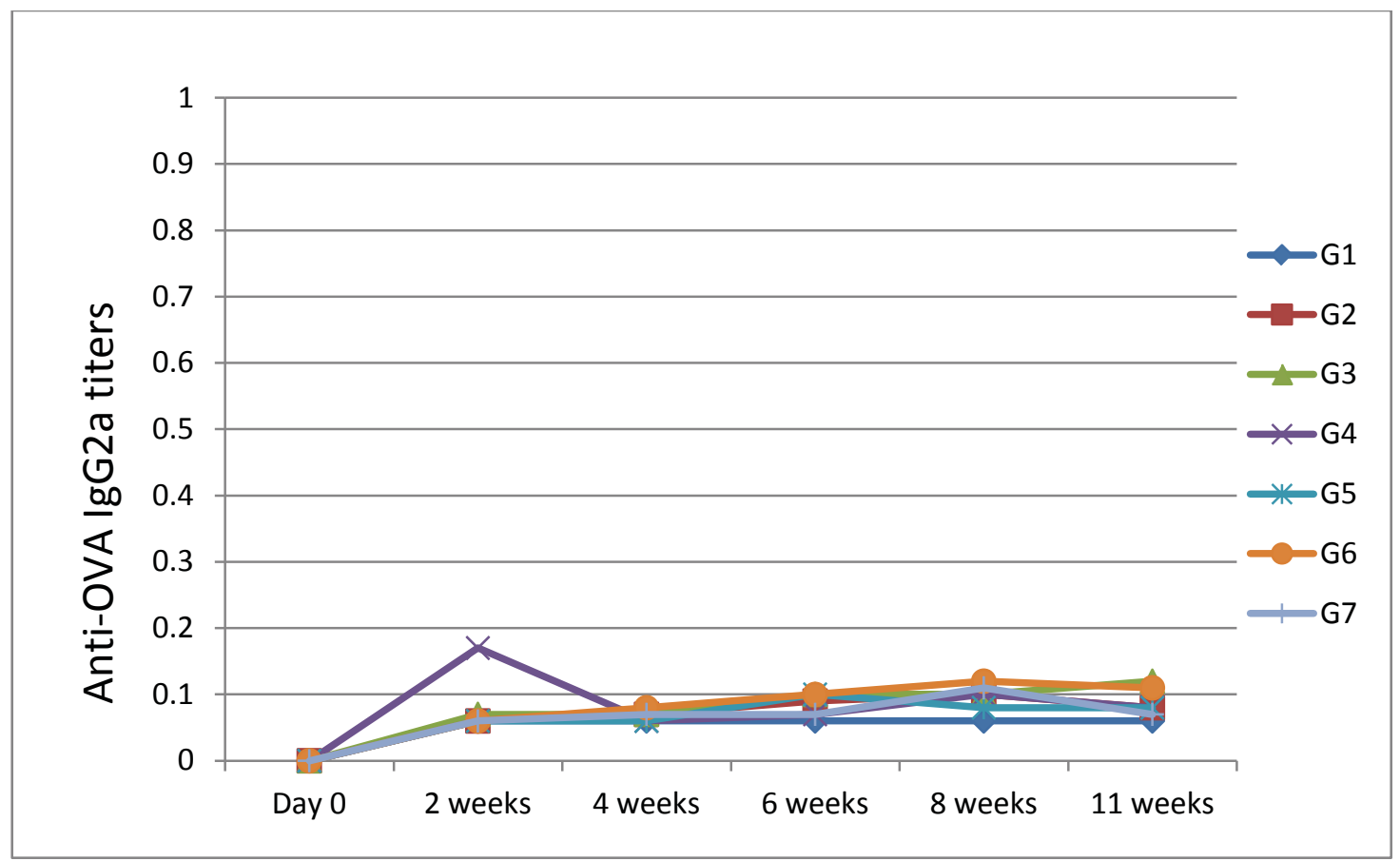

Figure 3-5. Mice serum anti-OVA IgG2a antibody titers.

G1, S.C. injection with PBS buffer and OVA; G2, S.C. injection ATBC emulsion and OVA; G3, S.C. injection with ALUM and OVA; G4, S.C. injection with AdjuGel 1 and OVA; G5, S.C. injection with AdjuGel 2 and OVA; G6, S.C. injection with AdjuGel 3 and OVA; G7, S.C. injection with AdjuGel 4 and OVA. 


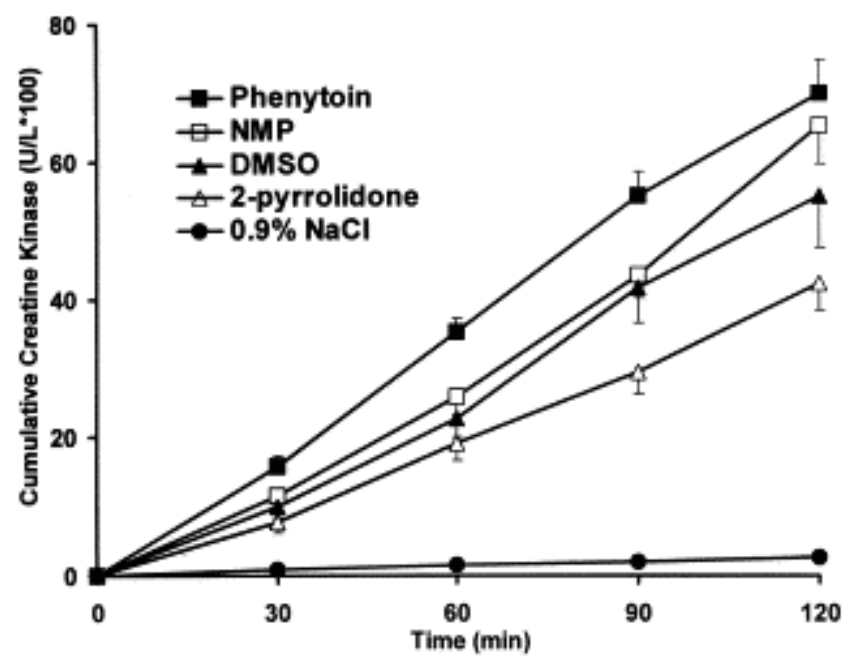

Figure 3-6. Cumulative CK-release following the injection of Nmethyl-2pyrrolidone (NMP), dimethylsulfoxide (DMSO), 2-pyrrolidone, phenytoin (positive control) and $0.9 \% \mathrm{NaCl}$ (negative control). (Reprinted with permission: Kranz, H., et al., Myotoxicity studies of injectable biodegradable in-situ forming drug delivery systems. Int J Pharm, 2001. 212(1): p. 11-8.) 
From the published data and our studies, it seems organic solvent NMP is relatively safe for big animals like monkey and rabbit, but can be toxic for small ones like rat and mouse. In the former studies we drew conclusion that organic solvent NMP is replaceable and other more hydrophobic solvents could be used in the AdjuGel system. (In Chapter 2) So we determined to find a safer and less irritating organic solvent for AdjuGel system.

According to FDA Q3C guideline,[55] organic solvent NMP belongs to class 2, which was defined as "non-genotoxic animal carcinogens or possible causative agents of irreversible toxicity, such as neurotoxicity or teratogenicity".[56] In order to decrease local inflammation and improve the biocompatibility, we started to screen the solvents in class 3 since they are much safer than the solvents in class 2 .

After carefully screening the organic solvent lists in class 3, ethyl acetate (EA) became a good choice of organic solvent since not only it could increase hydrophobicity but also decrease viscosity in AdjuGel system. In 2008, Rungseevijitprapa and his colleagues studied organic solvents ethyl acetate, benzyl alcohol, triethyl citrate, propylene carbonate and triacetin for the acute toxicity or tissue damage following intramuscular injection with rats.[51] They found the rank order of tissue damage of the solvents was ethyl acetate $<$ propylene carbonate $<$ triacetin $<$ triethyl citrate $<$ benzyl alcohol. (See Figure 3-7 below) Ethyl acetate showed low toxic potential, which is similar to isotonic normal saline $(0.9 \% \mathrm{NaCl})[51]$

Because of the data above, we determined to choose ethyl acetate instead of NMP in AdjuGel system for further studies.

Based on the results, we can clearly see that the AdjuGel system exhibited similar immune response profiles to that seen with the positive controls, Alum and ATBC emulsion. They all exhibited relatively high IgG1 titers but very low IgG2a titers, which suggest that the tested formulation can induce effective humoral immunity, but fail to induce good cell mediated immune responses, or suggest the absence of a T helper cell type 1 (Th1) response. Other immunopotentiators should be added if cell mediated immune responses are necessary.

\subsection{Conclusion}

From the data above, we concluded that: 1. Organic solvent NMP demonstrated toxicity to mice after subcutaneous injection and induced inflammation at the injection sites. 2. AdjuGel system, comprised of NMP, PLGA and ATBC, induce strong Th2 response with little or no Th1 response, which is similar to classic oil-based adjuvant and Alum adjuvant. 3. Although AdjuGel system, comprised of NMP, PLGA and ATBC, showed delayed release of ATBC in vitro, it didn't demonstrate delayed immune response in vivo. 


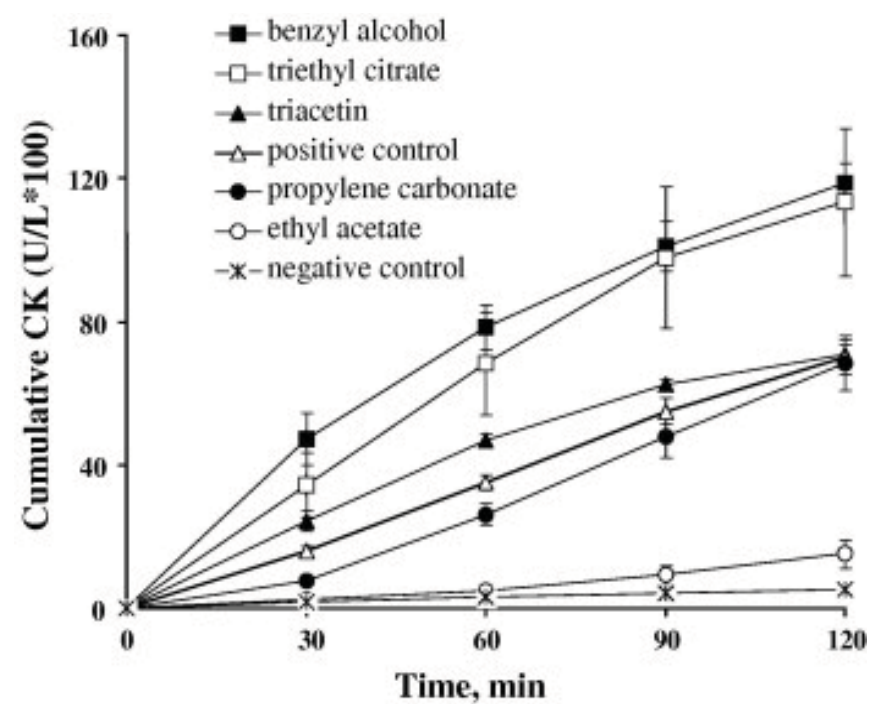

Figure 3-7. Cumulative $\mathrm{CK}$ release after $120 \mathrm{~min}$ following the injection of undiluted solvents in comparison to the positive control (phenytoin) and the negative control $(\mathbf{0 . 9 \%} \mathbf{~ N a C l})$. (Reprinted with permission: Rungseevijitprapa, W., et al., Myotoxicity studies of $\mathrm{O} / \mathrm{W}$-in situ forming microparticle systems. Eur J Pharm Biopharm, 2008. 69(1): p. 126-33.) 


\title{
CHAPTER 4. DEVELOPMENT OF NOVEL FORMULATIONS WITH DELAYED IMMUNE RESPONSE BASED ON ADJUGEL SYSTEM USING ORGANIC SOLVENT ETHYL ACETATE
}

\begin{abstract}
4.1. Introduction
Pulsatile release formulations for single dose vaccines have been studied for many years because of the advantages that they may simulate the vaccine administration with prime and booster shots. The most important key point in the formulation design is how to realize the delayed response and control the time window of booster response. General strategy was focused on programmed delay release of antigens in order to induce the delayed booster response. [47] Although the programmed delay release of antigens could be realized in vitro, the delayed release of antigens in vitro doesn't equal to delayed immune response in vivo. Until now, none of vaccine delivery systems achieved delayed immune response in vivo. We tried to approach this goal by designing new vaccine delivery system with delayed release of both antigen and vaccine adjuvant. Considering the functions of vaccine adjuvant in subunit vaccines, we believe that the delayed release of vaccine adjuvant is probably more important than the delayed release of antigen for delayed immune responses. In the AdjuGel system, the oil, ATBC and Hydrophobic polymer PLGA are the key components for immune response. Because ATBC is a plasticizer for PLGA polymer, it can interpose between individual strands of polymer to form a miscible condition with solidified polymer. Under such structure, the polymer can hold the plasticizer ATBC and ATBC won't be release until polymer starts to degrade. So the release of ATBC and PLGA is determined by the degradation of polymer PLGA. Organic solvent ethyl acetate is the ester of ethanol and acetic acid. It is a safe solvent with low toxicity. After intramuscular injection in rats, it showed similar acute toxicity or tissue damage to isotonic normal saline $(0.9 \% \mathrm{NaCl})$. [51] Additionally, ethyl acetate is relatively more hydrophobic solvent compared to NMP. Its solubility in water is 8.3 $\mathrm{g} / 100 \mathrm{~mL}$ in $20^{\circ} \mathrm{C}$ and NMP is miscible with water. The hydrophobicity of ethyl acetate may help to decrease the burst release of antigen proteins in the AdjuGel system. The burst release of antigen proteins may contribute to the fast response of AdjuGel groups in the previous experiment. Not only ethyl acetate may decrease the burst release of antigen proteins, but its hydrophobicity can also slow the degradation speed of PLGA polymer in AdjuGel system, which will stabilize AdjuGel system and elongate the delayed period before response.
\end{abstract}

\subsection{Experimental Procedures}

\subsubsection{Materials}

Twenty ICR female mice (6-8 weeks) were ordered from Harlan Laboratories. The biodegradable polymer PLGA and Polylactic acid (PLA) were ordered from Lactel Absorbable Polymers, Durect Corporation, Pelham, AL, USA. Ethyl acetate was ordered 
from Fisher Scientific; Acetyl Tributyl Citrate (ATBC) was obtained from Morflex Inc, Greensboro, NC, USA; Lyophilized Ovalbumin (OVA) as the antigen was purchased from Sigma.

Two PLGA polymers, PLGA (50:50, lactic acid: glycolic acid) and PLGA (85:15, lactic acid: glycolic acid) were used in the in vitro release study to compare the release profiles of OVA and ATBC. Polymer PLGA (50:50, lactic acid: glycolic acid) is relatively hydrophilic and polymer PLGA (85:15, lactic acid: glycolic acid) is relatively hydrophobic.

Besides two PLGA polymers, polymer PLGA (100:0, lactic acid: glycolic acid) or PLA was added in animal experiment to test the profiles of delayed immune response. Polymer PLA is the most hydrophobic polymer among the three polymers. Polylactic acid (PLA) is an aliphatic polyester. It is derived from renewable resources, such as corn starch, tapioca roots, chips or starch or sugarcane. When used in a drug delivery system, PLA has two different products: poly (L-lactide) and poly (DL-lactide). Poly (DL-lactide) is the polymer we planned to use. It is from Polymerization of a racemic mixture of L- and D-lactides and the approximately resorption time in vivo is 12 - 16 months. The chemical structure of poly (DL-lactide) is showed below (Figure 4-1).

Ethyl acetate is a colorless liquid has a characteristic sweet smell. It is an organic solvent with the formula $\mathrm{CH} 3-\mathrm{COO}-\mathrm{CH} 2-\mathrm{CH} 3$. In the experiment, we used ethyl acetate, instead of NMP as organic solvent in the AdjuGel system. The chemical structure of ethyl acetate is showed below (Figure 4-2).

\subsubsection{Methods}

Formulation process of the vaccines (all steps were prepared aseptically): 1 . Prepare three groups of AdjuGels consisting of a blend of PLGA or PLA and a combination of ethyl acetate and $\mathrm{ATBC}$ at $50^{\circ} \mathrm{C}-55^{\circ} \mathrm{C}$. 2. An appropriate quantity of gel was weighed and transferred in to an autoclaved clean glass vial. An appropriate quantity of OVA was weighed and blended with the gel and resulting mixture was stirred to obtain uniform mixture of OVA-loaded gel. 3. Each dose of OVA-loaded gel contains 100ug of OVA per $0.1 \mathrm{ml}$ gel.

\subsubsection{In vitro studies}

Two formulations of vaccine gel were prepared for in vitro release study. Formulation one used 65\% ethyl acetate (solvent), 20\% PLGA (50:50, IV: 0.65), 15\% ATBC and $1 \mathrm{mg} / \mathrm{ml}$ lyophilized ovalbumin. All ratios are weight to weight. Formulation two used 65\% ethyl acetate (solvent), 20\% PLGA (85:15, IV: 0.65), 15\% ATBC and $1 \mathrm{mg} / \mathrm{ml}$ lyophilized ovalbumin. The only difference between the two formulations is the polymers. Formulation one used a relatively hydrophilic polymer and formulation two 


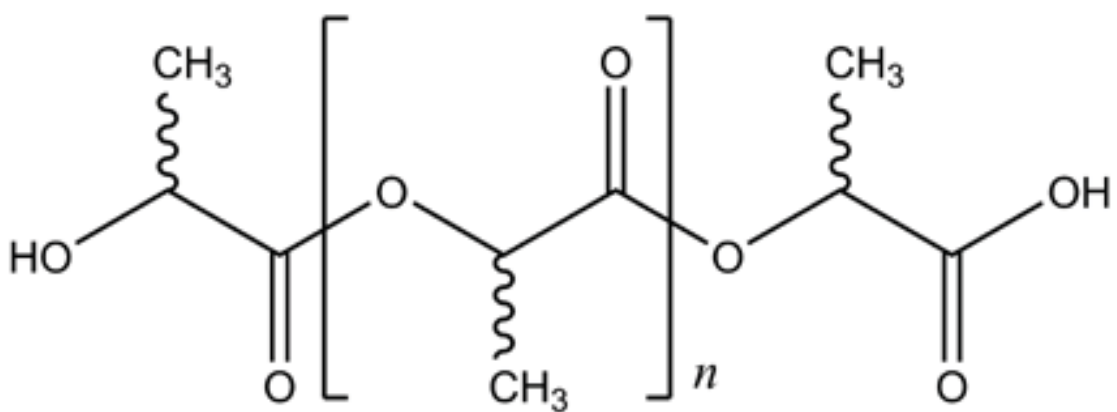

Figure 4-1. Chemical structure of poly (DL-lactide). 


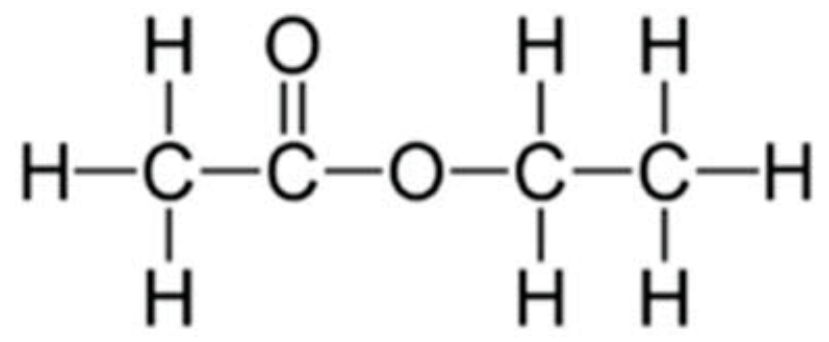

Figure 4-2. Chemical structure of ethyl acetate. 
used a relatively hydrophobic polymer.

$0.5 \mathrm{ml}$ of each vaccine gel (formulation one and two) was loaded into a bottle, and then $3.0 \mathrm{ml}$ PBS (pH 7.4, $0.01 \mathrm{M}$ ) was added. The sample bottles were kept in $37^{\circ} \mathrm{C}$ shaker at $60 \mathrm{rpm}$. At $24 \mathrm{hr}$ and then at two days and one week intervals, $2.0 \mathrm{ml}$ PBS sample was collected and analyzed for ovalbumin and ATBC concentration with Enzyme immunoassay (EIA) and HPLC method. $2.0 \mathrm{ml}$ fresh PBS was added after every collection. Release profiles were calculated by cumulative release with incubation time.

\subsubsection{Analytical method development for OVA (EIA)}

96-well microtiter plates (high protein binding plates from Costar) were coated overnight with $100 \mu \mathrm{l}$ of ovalbumin standard solutions and samples for test at $4{ }^{\circ} \mathrm{C}$. To remove unbound OVA, plates were washed three times with PBS ( $\mathrm{pH} 7.4)$ containing $0.05 \%$ Tween 20 (PBST). Primary antibody from rabbit were diluted in PBST and loaded into the plates with $100 \mu \mathrm{L} /$ well. The plates were then incubated for two hours at room temperature. The plates were again washed three times with PBST followed by addition of $100 \mu \mathrm{L}$ of PBST containing Horseradish Peroxidase (HRP) -conjugated goat antirabbit IgG (diluted 1:4000) (Abcam, ab6721). After a two hour incubation period at room temperature, the plates were washed three times with PBST followed by the addition of $200 \mu \mathrm{L}$ of $0.4 \mathrm{mg} / \mathrm{ml}$ OPD Peroxidase Substrate (Sigma P9187, Sigma-Aldrich, St. Louis, MO) and allowed to react for 30 minutes at room temperature. The optical density (OD) of the reaction was measured at $450 \mathrm{~nm}$ using a Plate Reader. The sample OVA concentrations were calculated in accordance to standard curve.

\subsubsection{Analytical method development for ATBC (HPLC)}

ATBC was analyzed by reverse phase (RP)-HPLC using a C18 column, $150 \mathrm{~mm}$ $\times 4.60 \mathrm{~mm}$ (SGE Analytical Science. Part No. 250112). The mobile phase was a 7:3 mixture of acetonitrile (HPLC grade) and water (HPLC grade). The flow rate was set at $1 \mathrm{ml} / \mathrm{min}$ and the injector volume was $50 \mu \mathrm{m}$. UV absorbance was measured at $234 \mathrm{~nm}$ using a photodiode array detector equipped with the high performance liquid chromatography system (HPLC). The results were calculated based on the readings from a standard series of ATBC.

\subsubsection{In vivo studies}

\subsubsection{Immunization of animals}

For all the immunization experiments, Lyophilized OVA protein was dissolved in PBS buffer or was suspended in different AdjuGel systems with organic solvent ethyl acetate immediately before injection to obtain a concentration of $1 \mathrm{mg} / \mathrm{ml}$. All mice were given single S.C. inoculation in neck site at day 0. Experimental assignment was shown in Table 4-1. 
Table 4-1. Mouse experimental group assignment.

\begin{tabular}{clcl}
\hline Group & Treatment & $\begin{array}{c}\text { Number of } \\
\text { mouse }\end{array}$ & Description \\
\hline 1 & Control & 5 & $\begin{array}{l}\text { S.C. injection with PBS buffer and OVA } \\
\text { S.C. injection with AdjuGel 1 and OVA }\end{array}$ \\
& $\begin{array}{l}\text { AdjuGel 1 } \\
\text { OVA }\end{array}$ & 5 & S.C. injection with AdjuGel 2 and OVA \\
AdjuGel 2 & 5 & S.C. injection with AdjuGel 3 and OVA \\
+OVA & $\begin{array}{l}\text { AdjuGel } 3 \\
\text { +OVA }\end{array}$ & 5 & \\
\hline
\end{tabular}

Ovalbumin is 50ug per injection. The volume is 50ul per injection.

AdjuGel 1: ethyl acetate $(65 \%, \mathrm{w} / \mathrm{w})$, ATBC $(15 \%, \mathrm{w} / \mathrm{w})$ and PLGA (50:50; IV: 0.65$)$.

AdjuGel 2: ethyl acetate $(65 \%, \mathrm{w} / \mathrm{w})$, ATBC $(15 \%, \mathrm{w} / \mathrm{w})$ and PLGA (85:15; IV: 0.65$)$.

AdjuGel 3: ethyl acetate $(65 \%, w / w)$, ATBC $(15 \%, w / w)$ and poly (DL-lactide) (IV: $0.65)$.

The PLGA concentration in AdjuGel 1, AdjuGel 2 and AdjuGel 3 is 20\% (w/w). 


\subsubsection{Procedures}

All groups were inoculated S. C. once at day 0 and blood samples were collected every 2 weeks in the first 12 weeks and every 3 or 4 weeks thereafter. Blood samples were serially diluted with $0.05 \%$ Tween 20 in PBS: 1:100, 1:1,000, 1:10,000 and 1:100,000. IgG titers were checked by ELISA (Enzyme-Linked Immunosorbant Assay); use Log10EC50 as a measure of immune response. EC50 means half maximal effective concentration and the absorbance of 1:100 dilution was used as the maximum.

\subsubsection{Enzyme linked immunosorbent assay (ELISA)}

96-well microtiter plates (high protein binding plates from Costar) were coated overnight with $100 \mu \mathrm{l}$ coating solution containing $3 \mu \mathrm{g} / \mathrm{mL}$ ovalbumin (OVA) at $4{ }^{\circ} \mathrm{C}$. To remove unbound OVA, plates were washed three times with PBS ( $\mathrm{pH} 7.4)$ containing $0.05 \%$ Tween 20 (PBST). Serum samples $(100 \mu \mathrm{L} /$ well) from individual mice were serially diluted in PBST: 1:100, 1:1,000, 1:10,000 and 1:100,000. The plates were then incubated for two hours at room temperature. The plates were again washed three times with PBST followed by addition of $100 \mu \mathrm{L}$ of PBST containing Horseradish Peroxidase (HRP) -conjugated Goat Anti-Mouse IgG (diluted 1:4000) (Abcam, ab6789). After a two hour incubation period at room temperature, the plates were washed three times with PBST followed by the addition of $200 \mu \mathrm{L}$ of $0.4 \mathrm{mg} / \mathrm{ml}$ OPD Peroxidase Substrate (Sigma P9187, Sigma-Aldrich, St. Louis, MO) and allowed to react for 30 minutes at room temperature. The optical density (OD) of the reaction was measured at $450 \mathrm{~nm}$ using a Plate Reader. Software BioDataFit was applied to deal with the data by the fourparameter model. Serum titers are reported with Log10EC50.

\subsubsection{Statistical analysis}

Results are expressed as mean \pm S.D. Statistical analysis was carried out for analyses of differences between means of serum antibody titers using Student's twotailed $t$-test. Differences in means were accepted as significant if $P$ was less than 0.05 .

\subsection{Results}

\subsubsection{In vitro release- Ovalbumin}

The study of OVA In vitro release showed that the two AdjuGel groups could provide continuous sustained release of OVA (Table 4-2 and Figure 4-3). In the groups of PLGA (50:50, IV: 0.65), OVA could be released up to eight weeks; in the groups of PLGA (85:15, IV: 0.65), OVA could be released up to fourteen weeks. The relatively hydrophobic PLGA polymer $(85: 15)$ could decrease initial release rate of OVA and prolong the whole release period of OVA. The relatively hydrophobic PLGA polymer 
Table 4-2. Data of in vitro OVA accumulative release with two groups.

\begin{tabular}{lcc}
\hline Time & G1(PLGA (50:50) & G2(PLGA(85:15) \\
\hline Day 0 & $0 \pm 0$ & $0 \pm 0$ \\
Day 1 & $0.06 \pm 0.004$ & $0.02 \pm 0.007$ \\
Day 4 & $0.1 \pm 0.008$ & $0.06 \pm 0.006$ \\
Week 1 & $0.13 \pm 0.007$ & $0.08 \pm 0.016$ \\
Week 2 & $0.19 \pm 0.009$ & $0.11 \pm 0.007$ \\
Week 3 & $0.26 \pm 0.009$ & $0.14 \pm 0.012$ \\
Week 4 & $0.32 \pm 0.008$ & $0.18 \pm 0.025$ \\
Week 5 & $0.48 \pm 0.023$ & $0.22 \pm 0.023$ \\
Week 6 & $0.71 \pm 0.053$ & $0.3 \pm 0.036$ \\
Week 7 & $0.87 \pm 0.027$ & $0.38 \pm 0.026$ \\
Week 8 & $1 \pm 0.039$ & $0.5 \pm 0.018$ \\
Week 9 & & $0.63 \pm 0.021$ \\
Week 10 & & $0.75 \pm 0.016$ \\
Week 11 & & $0.82 \pm 0.002$ \\
Week 12 & & $0.91 \pm 0.012$ \\
Week 13 & & $0.99 \pm 0.046$ \\
Week 14 & & $1 \pm 0.007$ \\
\hline
\end{tabular}

G1: $65 \%$ ethyl acetate (solvent), 20\% PLGA (50:50, IV: 0.65 ), $15 \%$ ATBC and $1 \mathrm{mg} / \mathrm{ml}$ lyophilized ovalbumin; G2: 65\% ethyl acetate (solvent), 20\% PLGA (85:15, IV: 0.65), $15 \%$ ATBC and $1 \mathrm{mg} / \mathrm{ml}$ lyophilized ovalbumin. All ratios are weight to weight. (Mean \pm S. D.) 


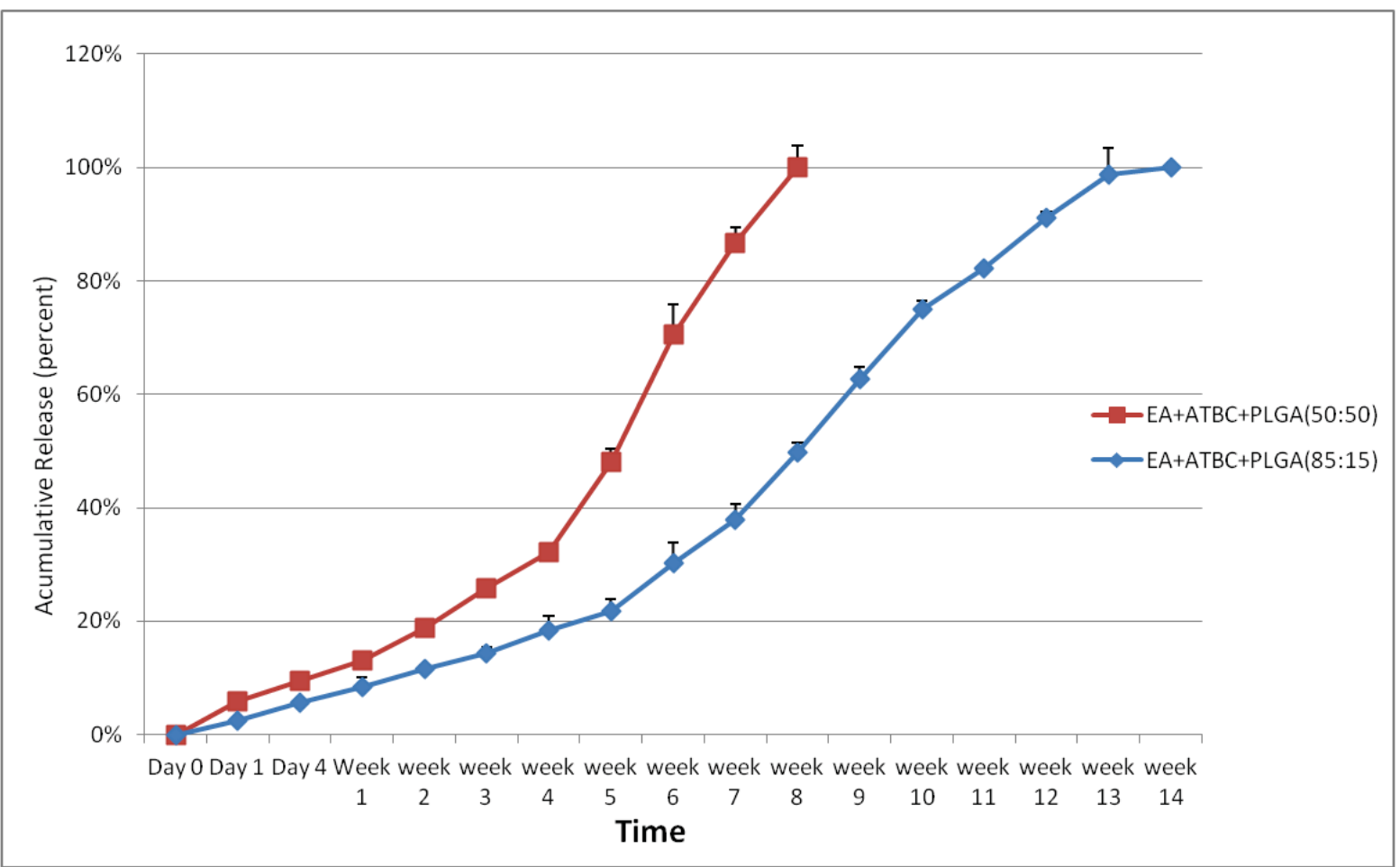

Figure 4-3. In vitro OVA accumulative release study with two groups.

Group 1: 65\% ethyl acetate (solvent), 20\% PLGA (50:50, IV: 0.65), 15\% ATBC and $1 \mathrm{mg} / \mathrm{ml}$ lyophilized ovalbumin; group two: $65 \%$ ethyl acetate (solvent), $20 \%$ PLGA (85:15, IV: 0.65$), 15 \%$ ATBC and $1 \mathrm{mg} / \mathrm{ml}$ lyophilized ovalbumin. All ratios are weight to weight. 
increased hydrophobicity of the AdjuGel system, which in turn decreases PBS buffer penetration into the gel and lower the OVA release rate.

There are no burst releases in the two groups. On day 4 , only $10 \%$ of total ovalbumin was released in formulation one. In previous study, $24 \%$ of total ovalbumin was released on day 3 with simailar formulation but using NMP as solvent. Also on day 4 , only $6 \%$ of total ovalbumin was released in formulation two. But in previous study, $14 \%$ of total ovalbumin was released on day 3 with simailar formulation but using NMP as solvent.

\subsubsection{In vitro release- ATBC}

The study of ATBC in vitro release showed that the both two groups could provide delayed release of ATBC (Table 4-3 and Figure 4-4). In the group of PLGA (50:50), ATBC wouldn't be released significantly until the sixth weeks; in the groups of PLGA (85:15), ATBC wouldn't be released significantly until the thirteenth weeks.

\subsubsection{In vivo study}

After S.C. injection at the neck sites, no skin lesions were found in all mice. But two mice showed hair loss in injection sites in group 2 (group AdjuGel 1). It's not clear what caused the hair loss, but the fast release of ethyl acetate by group AdjuGel 1 that contained relatively hydrophilic PLGA (50:50) may probably be related to the issue. The hair loss was completely recovered within three weeks without treatment.

Three different AdjuGel groups were tested and the results were shown in Table 4-4 and Figure 4-5. They have similar formulation, 65\% ethyl acetate (solvent), 20\% polymer, $15 \%$ ATBC and $1 \mathrm{mg} / \mathrm{ml}$ lyophilized ovalbumin. The only difference is the type of polymer.

In group of AdjuGel 1, relatively hydrophilic polymer PLGA (50:50) was used, which degraded fast and stimulated an immune response immediately. Statistical analysis showed the antibody titers of AdjuGel 1 are significant higher than the antibody titers of control group 1 at time points from week 2 to week $22(\mathrm{P}<0.01)$.

In group of AdjuGel 2, relatively hydrophobic polymer PLGA (85:15) was used, which degraded slowly and stimulated a delayed immune response at week 8 . Statistical analysis showed the antibody titers of AdjuGel 2 have no significant difference compared with the antibody titers of control group 1 at time points from day 0 to week $6(\mathrm{P}>0.05)$. Until week 8, the group of AdjuGel 2 stimulated a strong immune response. Statistical analysis showed the antibody titers of AdjuGel 2 are significant higher than the antibody titers of control group 1 at time points from week 8 to week $22(\mathrm{P}<0.01)$. 
Table 4-3. Data of in vitro ATBC accumulative release with two groups.

\begin{tabular}{lcc}
\hline Time & G1(PLGA (50:50) & G2(PLGA(85:15) \\
\hline Day 0 & $0 \pm 0$ & $0 \pm 0$ \\
Day 1 & $0.0128 \pm 0.0145$ & $0.0005 \pm 0.0006$ \\
Day 4 & $0.0131 \pm 0.0001$ & $0.0016 \pm 0.0005$ \\
Week 1 & $0.0137 \pm 0.0002$ & $0.0018 \pm 0.000035$ \\
Week 2 & $0.0149 \pm 0.0011$ & $0.0019 \pm 0.00003$ \\
Week 3 & $0.0151 \pm 0.0002$ & $0.0021 \pm 0.00002$ \\
Week 4 & $0.0164 \pm 0.0002$ & $0.0023 \pm 0.0001$ \\
Week 5 & $0.0167 \pm 0.00026$ & $0.0025 \pm 0.0001$ \\
Week 6 & $0.0925 \pm 0.0587$ & $0.0027 \pm 0.00008$ \\
Week 7 & $0.5774 \pm 0.164$ & $0.0029 \pm 0.00009$ \\
Week 8 & $1 \pm 0.07$ & $0.0031 \pm 0.0001$ \\
Week 9 & & $0.0032 \pm 0.00005$ \\
Week 10 & & $0.0034 \pm 0.00001$ \\
Week 11 & & $0.0036 \pm 0.00005$ \\
Week 12 & & $0.0039 \pm 0.0002$ \\
Week 13 & & $0.2582 \pm 0.145$ \\
Week 14 & & $1 \pm 0.169$ \\
\hline G1: 65\%
\end{tabular}

G1: $65 \%$ ethyl acetate (solvent), 20\% PLGA (50:50, IV: 0.65), 15\% ATBC and $1 \mathrm{mg} / \mathrm{ml}$ lyophilized ovalbumin; G2: 65\% ethyl acetate (solvent), 20\% PLGA (85:15, IV: 0.65), $15 \%$ ATBC and $1 \mathrm{mg} / \mathrm{ml}$ lyophilized ovalbumin. All ratios are weight to weight. (Mean \pm S. D.) 


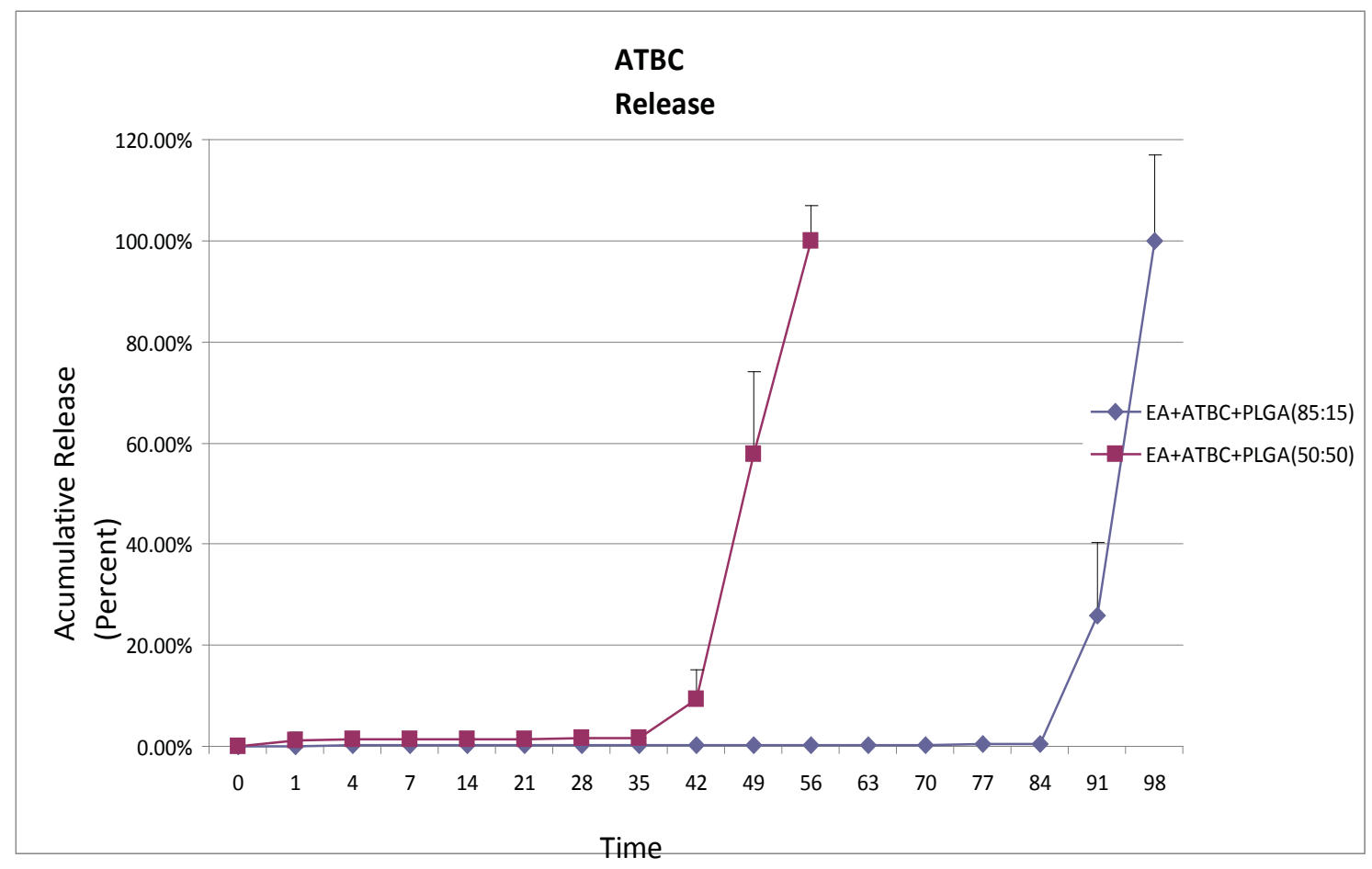

Figure 4-4. In vitro ATBC accumulative release study with two groups.

Group 1: 65\% ethyl acetate (solvent), 20\% PLGA (50:50, IV: 0.65), 15\% ATBC and $1 \mathrm{mg} / \mathrm{ml}$ lyophilized ovalbumin; group two: $65 \%$ ethyl acetate (solvent), $20 \%$ PLGA (85:15, IV: 0.65$), 15 \%$ ATBC and $1 \mathrm{mg} / \mathrm{ml}$ lyophilized ovalbumin. All ratios are weight to weight. 
Table 4-4. The results of serum anti-OVA IgG antibody titers for group 1, 2, 3 and 4 at different time points of day 0 , week $2,4,6,8,10,12,15,18$ and 22.

\begin{tabular}{lcccc}
\hline Time & G1 & G2 & G3 & G4 \\
\hline Day 0 & $0 \pm 0$ & $0 \pm 0$ & $0 \pm 0$ & $0 \pm 0$ \\
2 weeks & $0.14 \pm 0.01$ & $0.96 \pm 0.21$ & $0.19 \pm 0.05$ & $0.25 \pm 0.05$ \\
4 weeks & $0.11 \pm 0.01$ & $1.82 \pm 0.1$ & $0.31 \pm 0.14$ & $0.66 \pm 0.22$ \\
6 weeks & $0.16 \pm 0.04$ & $1.69 \pm 0.17$ & $0.63 \pm 0.51$ & $0.17 \pm 0.64$ \\
8 weeks & $0.13 \pm 0.01$ & $2.13 \pm 0.1$ & $1.79 \pm 0.42$ & $0.8 \pm 0.37$ \\
10 weeks & $0.15 \pm 0.04$ & $2.41 \pm 0.15$ & $1.24 \pm 0.41$ & $0.7 \pm 0.34$ \\
12 weeks & $0.2 \pm 0.02$ & $2.3 \pm 0.11$ & $1.44 \pm 0.63$ & $1.9 \pm 0.1$ \\
15 weeks & $0.23 \pm 0.03$ & $1.46 \pm 0.26$ & $1.01 \pm 0.45$ & $1 \pm 0.06$ \\
18 weeks & $0.21 \pm 0.08$ & $1.88 \pm 0.19$ & $1.33 \pm 0.5$ & $1.51 \pm 0.27$ \\
22 weeks & $0.15 \pm 0.03$ & $1.4 \pm 0.13$ & $1.15 \pm 0.22$ & $1.35 \pm 0.23$ \\
P value & & $0.005^{*}$ & $0.001 * *$ & $0.001 * * *$ \\
\hline
\end{tabular}

Serum anti-OVA total IgG titers were reported with Log10EC50 and results were expressed as Mean \pm S.D.

$\mathrm{P}$ values (*G2 vs. G1 with 2 weeks results; ** G3 vs. G1 with 8 weeks results; *** G4 vs. G1 with 12 weeks results) 


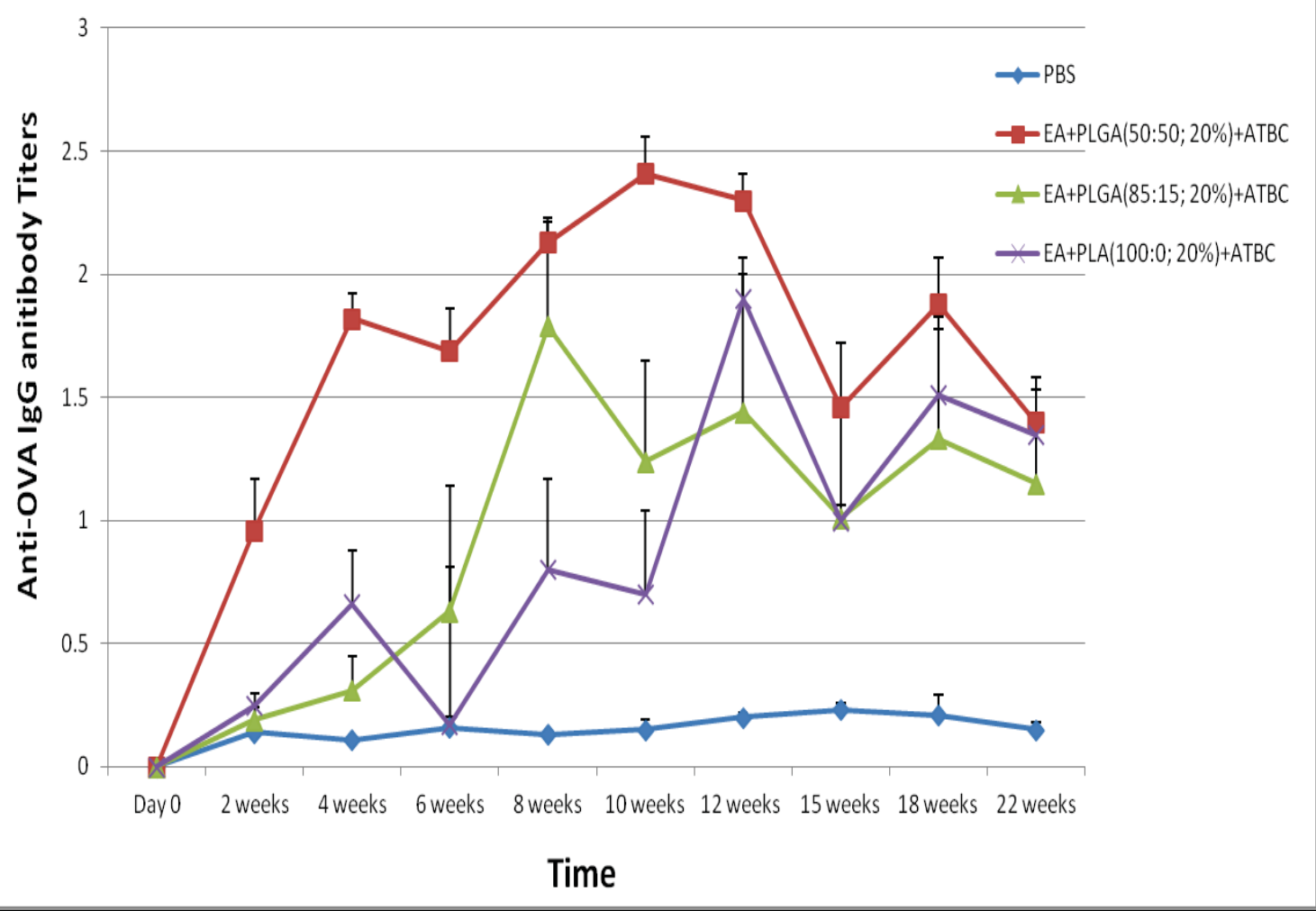

Figure 4-5. Mice serum anti-OVA IgG antibody titers.

Group 1, S.C. injection with PBS buffer and OVA; Group 2, S.C. injection with AdjuGel system containing ethyl acetate $(65 \%$, w/w), ATBC (15\%, w/w), PLGA (50:50; IV: $0.65)(20 \%$, w/w) and OVA; Group 3, S.C. injection with AdjuGel system containing ethyl acetate $(65 \%, \mathrm{w} / \mathrm{w})$, ATBC $(15 \%, \mathrm{w} / \mathrm{w})$, PLGA $(85: 15 ; \mathrm{IV}: 0.65)(20 \%, \mathrm{w} / \mathrm{w})$ and OVA; Group 4, S.C. injection with AdjuGel system containing ethyl acetate $(65 \%$, w/w $)$, ATBC $(15 \%, w / w)$, poly DL-lactide (IV: 0.65$)(20 \%, w / w)$ and OVA. 
In group of AdjuGel 3, the most hydrophobic polymer poly DL-lactide was used, which degraded very slow and the approximately degradation time in vivo is $12-16$ months. As a result, AdjuGel 3 group stimulated a delayed immune response at week 12. Statistical analysis showed the antibody titers of AdjuGel 3 have no significant difference compared with the antibody titers of control group 1 at time points from day 0 to week 6 $(\mathrm{P}>0.05)$. At week 8 and 10, although there is significant difference between the antibody titers of AdjuGel 3 and the antibody titers of control group 1, the values of the antibody titers of AdjuGel 3 are lower than 1 (0.7 and 0.8). Until week 12, the group of AdjuGel 3 stimulated a strong immune response. Statistical analysis showed the antibody titers of AdjuGel 3 are significant higher than the antibody titers of control group 1 at time points from week 12 to week $22(\mathrm{P}<0.01)$.

\subsection{Discussion}

New vaccine adjuvants or delivery systems for single dose vaccines have been studied for a long time. Although controlled release is believed critical for single dose vaccines, the correlation between patterns of controlled release and immune response was not clear. Strategy like PLGA microencapsulated antigen proteins was used for delayed release of antigens. Sanchez et al. reported PLGA microencapsulated Tetanus toxoid (TT) was programmed to release at two different time points (3 and 7 weeks) in vitro.[47] However, no successful in vivo data was published for the delayed immune response, which is probably because the delayed release of antigen proteins is not good enough for stimulation of an adaptive immune response in vivo.

Here we used a different strategy to achieve the goal of delayed immune response. We did not only focus on the controlled release of antigen, but also on the degradation and interaction of polymer and surfactant to delay the onset of adjuvanticity in AdjuGel system. First, combination of hydrophobic polymer PLGA and plasticizer ATBC (AdjuGel system) was found capable to induce strong immune response. After several studies on functions of the individual component (solvent, polymer and plasticizer), it's clear that only the combination of hydrophobic polymer PLGA and plasticizer ATBC could show effective adjuvanticity, any individual component cannot induce effective response. The combination of hydrophobic polymer PLGA and plasticizer ATBC is similar to the classic oil based vaccine adjuvants, which require the combination of surfactants and oil. By comparing the two types of vaccine adjuvants and their modes of action, we hypothesize the degradation of polymer may be a critical prerequisite for the adjuvanticity of AdjuGel system.

Then we performed an unsuccessful animal experiment that did not show any delayed immune response. From the failed experiment, we learned that not only polymer PLGA and ATBC, but solvent also determined the polymer degradation rate in vivo. Organic solvent NMP may accelerate the degradation of polymer PLGA by two possibilities: one is the irritation of NMP causes the local inflammation, which in turn stimulates innate immunity; another one is the hydrophilicity of NMP enhances the penetration of non-solvent (water), which also accelerates the PLGA degradation. 
Later, after scrutinizing and evaluating the available organic solvents, we chose ethyl acetate to replace NMP. Organic solvent ethyl acetate is much less irritating and more hydrophobic than NMP. Finally, the new formulation showed adjustable delayed immune responses, which is compatible with the degradation rates of various polymers. These results proved that our new strategy worked very well for development of new vaccine formulation with adjustable delayed immune response.

There are other advantages for the new vaccine formulation. In the new formulation, we used lyophilized antigen proteins. The lyophilized proteins are very stable in the gel solution of ethyl acetate, PLGA and ATBC because they cannot dissolve in the gel solution and are kept in solid state. At the same time, the gel solution prevents the protein from contact with aqueous surrounding. So the new vaccine formulation may be used for the preparation of vaccines that do not need refrigeration. Thermostable vaccines will dramatically reduce the cost of cold chain, which consumes about $80 \%$ of the total cost of vaccination programs.[57]

Another advantage is the new vaccine formulation can hold multiple vaccine antigens. Antigen proteins are suspended in the AdjuGel system, but not conjugated or attached to polymer. So the solid particles of antigen proteins will be separated and will not affect each other. By this way, the AdjuGel system may help to prepare polyvalent vaccine like MMR vaccine, which immunizes against three viruses - the viruses that cause measles, mumps, and rubella. Polyvalent vaccines can save cost for prophylactic vaccines. Also polyvalent vaccines may be more effective for therapeutic vaccines like contraceptive vaccine and cancer vaccine.

\subsection{Conclusion}

Our data above indicated that: 1. Organic solvent ethyl acetate was much less toxic to mice after subcutaneous injection compared with NMP. 2. AdjuGel 1 group, composed of ethyl acetate, ATBC and PLGA (50:50, IV: 0.65) stimulated a fast immune response after a single subcutaneous injection. 3. AdjuGel 2 group, composed of ethyl acetate, ATBC and PLGA (85:15, IV: 0.65) stimulated a delayed immune response on week 8 after a single subcutaneous injection. 4. AdjuGel 3 group, composed of ethyl acetate, ATBC and poly DL-lactide (IV: 0.65) stimulated a delayed immune response on week 12 after a single subcutaneous injection. 5. The delayed period for immune response was adjustable, which was determined by the degradation rates of polymers. The lower the polymer degradation rate, the longer the delayed period for immune response. 


\section{CHAPTER 5. DEVELOPMENT OF SINGLE DOSE VACCINE WITH THE ADJUGEL SYSTEM}

\subsection{Introduction}

After preparation of the new vaccine formulations with adjustable delayed immune response from the prior section, a single dose vaccine could be developed by two simultaneous separate inoculations. One used the formulation of group AdjuGel 1 (see last mouse experiment), which can provide prime or immediate response; another one used formulation of group AdjuGel 2 (see last mouse experiment), which will provide a booster or delayed response. We assumed two simultaneous separate inoculations may mimic two sequential shots that render clear prime response and booster response

\subsection{Experimental Procedures}

\subsubsection{Materials}

Twenty ICR female mice (6-8 weeks) were ordered from Harlan Laboratories. The biodegradable polymer PLGA were ordered from Lactel Absorbable Polymers, Durect Corporation, Pelham, AL, USA. Ethyl acetate was ordered from Fisher Scientific; Acetyl Tributyl Citrate (ATBC) was obtained from Morflex Inc, Greensboro, NC, USA; Lyophilized Ovalbumin (OVA) as the antigen was purchased from Sigma.

\subsubsection{Immunization of animals}

For all the immunization experiments, Lyophilized OVA protein was suspended in different AdjuGel systems with organic solvent ethyl acetate immediately before injection to obtain a concentration of $1 \mathrm{mg} / \mathrm{ml}$. The mice of group 4 were given two simultaneous separate inoculations: inoculation one used formulation of group AdjuGel 1 (see last mouse experiment) and was injected subcutaneously at the neck site with 50ug OVA per injection of 50ul; inoculation two used formulation of group AdjuGel 2 (see last mouse experiment) and was injected subcutaneously at the site of lower back with 50ug OVA per injection of 50ul. All other mice were given single S.C. inoculation at the site of neck area with 50ug OVA per injection of 50ul. Experimental assignment was shown in Table 5-1.

\subsubsection{Procedures}

All groups were inoculated S. C. once at day 0, and blood samples were collected every 2 weeks in the first 12 weeks and every 3 or 4 weeks thereafter and serially diluted with $0.05 \%$ Tween 20 in PBS: 1:100, 1:1,000, 1:10,000 and 1:100,000. IgG titers were 
Table 5-1. Experimental group assignment with two-injections.

\begin{tabular}{clcl}
\hline Group & Treatment & $\begin{array}{c}\text { Number } \\
\text { of mouse }\end{array}$ & Description \\
\hline 1 & Control & 5 & S.C. injection with PBS buffer and OVA \\
2 & AdjuGel 1 + OVA & 5 & S.C. injection with AdjuGel 1 and OVA \\
3 & AdjuGel 2 +OVA & 5 & S.C. injection with AdjuGel 2 and OVA \\
4 & $\begin{array}{l}\text { (AdjuGel 1 +OVA) } \\
\text { Plus (AdjuGel 2 }\end{array}$ & 5 & $\begin{array}{l}\text { S.C. injection with (AdjuGel 1 +OVA) Plus } \\
\text { (AdjuGel 2 +OVA) }\end{array}$ \\
\begin{tabular}{l} 
+OVA) \\
\hline
\end{tabular}
\end{tabular}

Ovalbumin is 50ug per injection. The volume is $50 \mathrm{ul}$ per injection.

AdjuGel 1: ethyl acetate (65\%, w/w), ATBC (15\%, w/w) and PLGA (50:50; IV: 0.65). AdjuGel 2: ethyl acetate $(65 \%, \mathrm{w} / \mathrm{w})$, ATBC $(15 \%, \mathrm{w} / \mathrm{w})$ and PLGA $(85: 15 ;$ IV: 0.65$)$. The PLGA concentration in AdjuGel 1 and AdjuGel 2 is $20 \%(\mathrm{w} / \mathrm{w})$. 
checked by ELISA (Enzyme-Linked Immunosorbant Assay); use Log10EC50 as a measure of immune response. EC50 means half maximal effective concentration and the absorbance of 1:100 dilution was used as the maximum.

\subsubsection{Enzyme linked immunosorbent assay (ELISA)}

96-well microtiter plates (high protein binding plates from Costar) were coated overnight with $100 \mu \mathrm{l}$ coating solution containing $3 \mu \mathrm{g} / \mathrm{mL}$ ovalbumin (OVA) at $4{ }^{\circ} \mathrm{C}$. To remove unbound OVA, plates were washed three times with PBS (pH 7.4) containing $0.05 \%$ Tween 20 (PBST). Serum samples $(100 \mu \mathrm{L} /$ well) from individual mice were serially diluted in PBST: 1:100, 1:1,000, 1:10,000 and 1:100,000. The plates were then incubated for two hours at room temperature. The plates were again washed three times with PBST followed by addition of $100 \mu \mathrm{L}$ of PBST containing Horseradish Peroxidase (HRP) -conjugated Goat Anti-Mouse IgG (diluted 1:4000) (Abcam, ab6789). After a two hour incubation period at room temperature, the plates were washed three times with PBST followed by the addition of $200 \mu \mathrm{L}$ of $0.4 \mathrm{mg} / \mathrm{ml}$ OPD Peroxidase Substrate (Sigma P9187, Sigma-Aldrich, St. Louis, MO) and allowed to react for 30 minutes at room temperature. The optical density (OD) of the reaction was measured at $450 \mathrm{~nm}$ using a Plate Reader. Software BioDataFit was applied to deal with the data by the fourparameter model. Serum titers are reported with Log10EC50.

\subsubsection{Statistical analysis}

Results are expressed as mean \pm S.D. Statistical analysis was carried out for analyses of differences between means of serum antibody titers using Student's twotailed $t$-test. Differences in means were accepted as significant if $P$ was less than 0.05 .

\subsection{Results}

After S.C. injection, some mice in group 4 showed mild to moderate skin lesions (local inflammatory reaction at the injection sites). The reason is not very clear. It may be related to over dose of ethyl acetate or the joint effects of two simultaneous separate inoculations. The skin lesions were recovered within three weeks after treatment with antibiotic ointment.

After two simultaneous inoculations, group 4 showed a fast and strong immune response and seemed to show a booster response at week 12 (Table 5-2 and Figure 5-1). However, the level of immune response had no significant difference with the level of immune response in group 2 (control group) $(\mathrm{P}>0.05)$, which means the booster response is not confirmed. 
Table 5-2. The results of serum anti-OVA IgG antibody titers for group 1, 2, 3 and 4 at different time points of day 0 , week $2,4,6,8,10,12,15,18$ and 22.

\begin{tabular}{lcccc}
\hline Time & G1 & G2 & G3 & G4 \\
\hline Day 0 & $0 \pm 0$ & $0 \pm 0$ & $0 \pm 0$ & $0 \pm 0$ \\
2 weeks & $0.14 \pm 0.01$ & $0.96 \pm 0.21$ & $0.19 \pm 0.05$ & $1.07 \pm 0.15$ \\
4 weeks & $0.11 \pm 0.01$ & $1.82 \pm 0.1$ & $0.31 \pm 0.14$ & $2.15 \pm 0.53$ \\
6 weeks & $0.16 \pm 0.04$ & $1.69 \pm 0.17$ & $0.63 \pm 0.51$ & $2.18 \pm 0.15$ \\
8 weeks & $0.13 \pm 0.01$ & $2.13 \pm 0.1$ & $1.79 \pm 0.42$ & $1.94 \pm 0.34$ \\
10 weeks & $0.15 \pm 0.04$ & $2.41 \pm 0.15$ & $1.24 \pm 0.41$ & $1.98 \pm 0.18$ \\
12 weeks & $0.2 \pm 0.02$ & $2.3 \pm 0.11$ & $1.44 \pm 0.63$ & $2.44 \pm 0.24$ \\
15 weeks & $0.23 \pm 0.03$ & $1.46 \pm 0.26$ & $1.01 \pm 0.45$ & $1.68 \pm 0.08$ \\
18 weeks & $0.21 \pm 0.08$ & $1.88 \pm 0.19$ & $1.33 \pm 0.5$ & \\
22 weeks & $0.15 \pm 0.03$ & $1.4 \pm 0.13$ & $1.15 \pm 0.22$ & \\
P value & & & & \\
(12 weeks) & & & & $0.30^{*}$ \\
\hline
\end{tabular}

Serum anti-OVA IgG titers were reported with Log10EC50 and results were expressed as Mean \pm S.D.

$P$ value with 12 weeks results (* G4 vs. G2) 


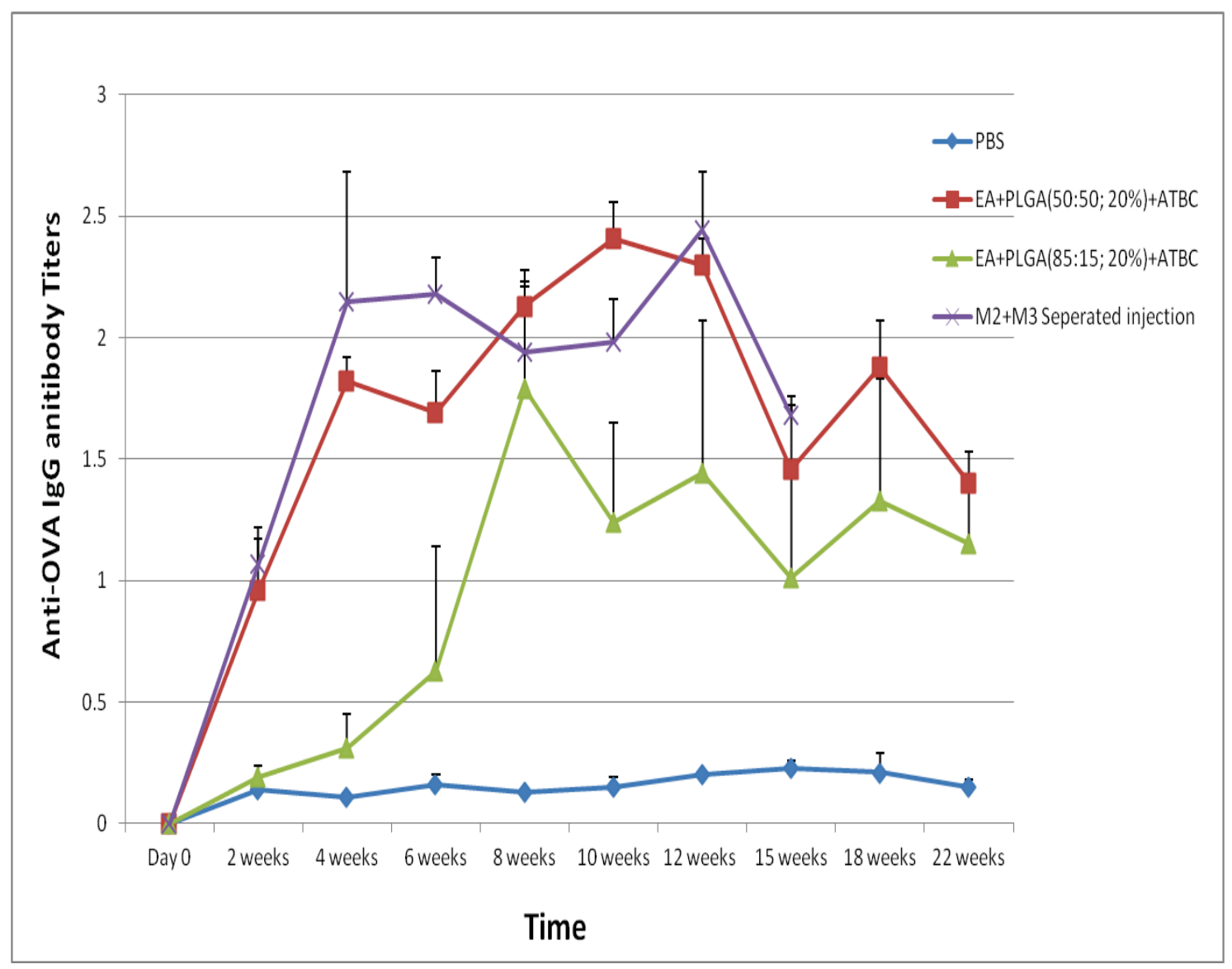

Figure 5-1. Mice serum anti-OVA IgG antibody titers with two-injection group. Group 1, S.C. injection with PBS buffer and OVA; Group 2, S.C. injection with AdjuGel system containing ethyl acetate $(65 \%$, w/w), ATBC $(15 \%$, w/w), PLGA (50:50; IV: $0.65)(20 \%$, w/w) and OVA; Group 3, S.C. injection with AdjuGel system containing ethyl acetate $(65 \%, \mathrm{w} / \mathrm{w})$, ATBC $(15 \%, \mathrm{w} / \mathrm{w})$, PLGA $(85: 15 ; \mathrm{IV}: 0.65)(20 \%$, w/w) and OVA; Group 4, S.C. injection with Group 2 and Group 3 simultaneously, one at the site of neck area and another one at the site of lower back. 


\subsection{Discussion}

This experiment didn't clearly demonstrate two peaks with respective prime and booster response. There are several possibilities to explain the phenomenon. Firstly, the two simultaneous inoculations may affect each other and the immediate response may have inhibited the booster response. Secondly, the prime stimulation may be too strong, which may have covered the booster response from the formulation of AdjuGel 2.

Further study is needed for optimization of the formulations by designing the onset of delayed immune response and to find the best combination of different AdjuGel formulations. Also other immunopotentiators could be added to AdjuGel for better outcome.

\subsection{Conclusion}

Two simultaneous inoculations induced a fast and strong immune response at week 4 but an equivocal booster response at week 12. Further optimization of AdjuGel formulations is needed for development of single dose vaccine. 


\section{CHAPTER 6. RESEARCH SUMMARY}

A novel vaccine adjuvant or vaccine delivery system, named AdjuGel system, has been developed for single dose vaccines with the characteristics of adjustable delayed immune response.

Formulations for single dose vaccines have be studied for long time. However, until now no successful formulation or mechanism was developed. We have designed new vaccine adjuvant formulations by focusing on the booster immune response. Based on In Situ Implant (ISI) system, the AdjuGel system was designed as a new vaccine adjuvant formulation, which was composed of organic solvent NMP, hydrophobic plasticizer ATBC and polymer PLGA. After studies in animal experiments, hydrophobic plasticizer ATBC and polymer PLGA were confirmed to be the two key components for effective immune responses in the AdjuGel system.

The oil-based formulations are probably the most classic formulations in vaccine adjuvants. There are also two key components in oil-based vaccine adjuvant formulations, hydrophobic oil and surfactant. When compared the adjuGel system to the oil-based vaccine adjuvant, they displayed similar mode of action: the combination of two key components is necessary for immunogenicity. Since the hydrophobic plasticizer ATBC is an oil, we hypothesized polymer PLGA not only acted as a high molecular weight polymer for sustained release, but also acted as a surfactant in the AdjuGel system. However, hydrophobic polymer PLGA with high molecular weight will not act as a surfactant before its degradation. After a period of time for degradation, long polymer chains will be degraded into shorter ones. As a result, the reduction in molecular weight produces an increase in hydrophilicity. When the hydrophilicity lipophilicity balance (HLB) of polymer chains reach a suitable range, hydrophobic polymer PLGA or PLA will show the characteristics of surfactants, which in turn may alter the AdjuGel system to be more similar to the classic oil-based vaccine adjuvant and induce immune response.

Based on the hypothesis above, the AdjuGel system, composed of NMP, ATBC and PLGA, was tested in an animal experiment. The results didn't show any delayed immune response, only showed immediate response with local inflammation at the injection sites. We realized that probably the irritation and hydrophilicity of NMP caused the accelerated degradation rate and immediate response.

Since the organic solvent ethyl acetate is less irritating and more hydrophobic than NMP, it was chosen to replace NMP. The new AdjuGel system is a novel system composed of ethyl acetate, ATBC and PLGA. It did show modified component release and adjustable delayed immune responses, which are compatible with the degradation rates of various polymers. These results confirmed that our new strategy may work for development of single dose vaccine formulations. 


\section{LIST OF REFERENCES}

1. Wilson-Welder, J.H., et al., Vaccine adjuvants: current challenges and future approaches. J Pharm Sci, 2009. 98(4): p. 1278-316.

2. Plotkin, S.A. and W.A. Orenstein, Vaccines. Fourth ed. 2004, Philadelphia: Elsevier Inc. 1662.

3. Freund J, C.J., Hosmer E, Sensitization and antibody formation after injectin of tubercle bacilli and parafin oil. Proc Soc Exp Biol Med, 1937. 37: p. 509-13

4. Freund, J., The effect of paraffin oil and mycobacteria on antibody formation and sensitization; a review. Am J Clin Pathol, 1951. 21(7): p. 645-56.

5. Herbert, W.J., The mode of action of mineral-oil emulsion adjuvants on antibody production in mice. Immunology, 1968. 14(3): p. 301-18.

6. O'Hagan, D.T. and R. Rappuoli, Novel approaches to vaccine delivery. Pharm Res, 2004. 21(9): p. 1519-30.

7. Brito, L.A., P. Malyala, and D.T. O'Hagan, Vaccine adjuvant formulations: a pharmaceutical perspective. Semin Immunol, 2013. 25(2): p. 130-45.

8. Calabro, S., et al., The adjuvant effect of MF59 is due to the oil-in-water emulsion formulation, none of the individual components induce a comparable adjuvant effect. Vaccine, 2013. 31(33): p. 3363-9.

9. $\quad$ Preis, I. and R.S. Langer, A single-step immunization by sustained antigen release. J Immunol Methods, 1979. 28(1-2): p. 193-7.

10. O'Hagan, D.T., et al., Biodegradable microparticles as controlled release antigen delivery systems. Immunology, 1991. 73(2): p. 239-42.

11. Alonso, M.J., et al., Biodegradable microspheres as controlled-release tetanus toxoid delivery systems. Vaccine, 1994. 12(4): p. 299-306.

12. Langer, R., J.L. Cleland, and J. Hanes, New advances in microsphere-based single-dose vaccines. Adv Drug Deliv Rev, 1997. 28(1): p. 97-119.

13. Gupta, R.K., A.C. Chang, and G.R. Siber, Biodegradable polymer microspheres as vaccine adjuvants and delivery systems. Dev Biol Stand, 1998. 92: p. 63-78.

14. Jiang, W. and S.P. Schwendeman, Stabilization of a model formalinized protein antigen encapsulated in poly(lactide-co-glycolide)-based microspheres. J Pharm Sci, 2001. 90(10): p. 1558-69.

15. Cui, C., V.C. Stevens, and S.P. Schwendeman, Injectable polymer microspheres enhance immunogenicity of a contraceptive peptide vaccine. Vaccine, 2007.

25(3): p. 500-9.

16. Jiang, W., et al., Biodegradable poly(lactic-co-glycolic acid) microparticles for injectable delivery of vaccine antigens. Adv Drug Deliv Rev, 2005. 57(3): p. 391410.

17. Oyewumi, M.O., A. Kumar, and Z. Cui, Nano-microparticles as immune adjuvants: correlating particle sizes and the resultant immune responses. Expert Rev Vaccines, 2010. 9(9): p. 1095-107.

18. Conway, M.A., et al., Protection against Bordetella pertussis infection following parenteral or oral immunization with antigens entrapped in biodegradable particles: effect of formulation and route of immunization on induction of Th1 and Th2 cells. Vaccine, 2001. 19(15-16): p. 1940-50. 
19. Leong, K.W., et al., Bioerodible polyanhydrides as drug-carrier matrices. II. Biocompatibility and chemical reactivity. J Biomed Mater Res, 1986. 20(1): p. 51-64.

20. Torres, M.P., et al., Amphiphilic polyanhydrides for protein stabilization and release. Biomaterials, 2007. 28(1): p. 108-16.

21. Tabata, Y., S. Gutta, and R. Langer, Controlled delivery systems for proteins using polyanhydride microspheres. Pharm Res, 1993. 10(4): p. 487-96.

22. Determan, A.S., et al., Protein stability in the presence of polymer degradation products: consequences for controlled release formulations. Biomaterials, 2006. 27(17): p. 3312-20.

23. Phanse, Y., et al., Functionalization of polyanhydride microparticles with dimannose influences uptake by and intracellular fate within dendritic cells. Acta Biomater, 2013. 9(11): p. 8902-9.

24. Shu, Q., et al., Effects of various adjuvants on efficacy of a vaccine against Streptococcus bovis and Lactobacillus spp in cattle. Am J Vet Res, 2000. 61(7): p. 839-43.

25. Amidi, M., et al., Diphtheria toxoid-containing microparticulate powder formulations for pulmonary vaccination: preparation, characterization and evaluation in guinea pigs. Vaccine, 2007. 25(37-38): p. 6818-29.

26. Heritage, P.L., et al., Novel polymer-grafted starch microparticles for mucosal delivery of vaccines. Immunology, 1996. 88(1): p. 162-8.

27. Suckow, M.A., et al., Oral immunization of rabbits against Pasteurella multocida with an alginate microsphere delivery system. J Biomater Sci Polym Ed, 1996. 8(2): p. 131-9.

28. O'Hagan, D.T. and E. De Gregorio, The path to a successful vaccine adjuvant'the long and winding road'. Drug discovery today, 2009. 14(11): p. 541-551.

29. Arya, S.C., Human immunization in developing countries: practical and theoretical problems and prospects. Vaccine, 1994. 12(15): p. 1423-35.

30. http://www.grandchallenges.org/Pages/BrowseByGoal.aspx.

31. Bowersock, T.L. and S. Martin, Vaccine delivery to animals. Adv Drug Deliv Rev, 1999. 38(2): p. 167-194.

32. Men, Y., et al., Induction of a cytotoxic T lymphocyte response by immunization with a malaria specific CTL peptide entrapped in biodegradable polymer microspheres. Vaccine, 1997. 15(12-13): p. 1405-12.

33. Moore, A., et al., Immunization with a soluble recombinant HIV protein entrapped in biodegradable microparticles induces HIV-specific CD8+ cytotoxic T lymphocytes and CD4+ Th1 cells. Vaccine, 1995. 13(18): p. 1741-9.

34. Nixon, D.F., et al., Synthetic peptides entrapped in microparticles can elicit cytotoxic T cell activity. Vaccine, 1996. 14(16): p. 1523-30.

35. Gupta, R.K., et al., Chronic local tissue reactions, long-term immunogenicity and immunologic priming of mice and guinea pigs to tetanus toxoid encapsulated in biodegradable polymer microspheres composed of poly lactide-co-glycolide polymers. Vaccine, 1997. 15(16): p. 1716-23.

36. Dunn, R.L., English J.P., Cowsar, D.R., Vanderbilt,D,P., Biodegradable in situ forming implants and methods of producing the same. 1990: US. 
37. Graham, P.D., K.J. Brodbeck, and A.J. McHugh, Phase inversion dynamics of PLGA solutions related to drug delivery. J Control Release, 1999. 58(2): p. 23345.

38. Dunn, R.L., Tipton, A.J., Polymeric compositions useful as controlled release implants. 1997, Atrix lab, Inc: US.

39. McHugh, A.J., The role of polymer membrane formation in sustained release drug delivery systems. J Control Release, 2005. 109(1-3): p. 211-21.

40. Krebs, M.D., et al., Injectable poly(lactic-co-glycolic) acid scaffolds with in situ pore formation for tissue engineering. Acta Biomater, 2009. 5(8): p. 2847-59.

41. Packhaeuser, C.B., et al., In situ forming parenteral drug delivery systems: an overview. Eur J Pharm Biopharm, 2004. 58(2): p. 445-55.

42. Men, Y., et al., A single administration of tetanus toxoid in biodegradable microspheres elicits $T$ cell and antibody responses similar or superior to those obtained with aluminum hydroxide. Vaccine, 1995. 13(7): p. 683-9.

43. Tabata, Y. and Y. Ikada, Macrophage phagocytosis of biodegradable microspheres composed of L-lactic acid/glycolic acid homo- and copolymers. J Biomed Mater Res, 1988. 22(10): p. 837-58.

44. Shideler, S.E., et al., Use of porcine zona pellucida (PZP) vaccine as a contraceptive agent in free-ranging tule elk (Cervus elaphus nannodes). Reprod Suppl, 2002. 60: p. 169-76.

45. Kirkpatrick, J.F., et al., Long-term effects of porcine zonae pellucidae immunocontraception on ovarian function in feral horses (Equus caballus). J Reprod Fertil, 1992. 94(2): p. 437-44.

46. Vicente, S., et al., A polymer/oil based nanovaccine as a single-dose immunization approach. PLoS One, 2013. 8(4): p. e62500.

47. Sanchez, A., et al., Pulsed controlled-released system for potential use in vaccine delivery. J Pharm Sci, 1996. 85(6): p. 547-52.

48. Godavarthy, S.S., et al., Design of improved permeation enhancers for transdermal drug delivery. J Pharm Sci, 2009. 98(11): p. 4085-99.

49. Lee, P.J., R. Langer, and V.P. Shastri, Role of n-methyl pyrrolidone in the enhancement of aqueous phase transdermal transport. J Pharm Sci, 2005. 94(4): p. 912-7.

50. Kranz, H., et al., Myotoxicity studies of injectable biodegradable in-situ forming drug delivery systems. Int J Pharm, 2001. 212(1): p. 11-8.

51. Rungseevijitprapa, W., et al., Myotoxicity studies of $\mathrm{O} / W$-in situ forming microparticle systems. Eur J Pharm Biopharm, 2008. 69(1): p. 126-33.

52. Singh, M. and D.T. O'Hagan, Recent advances in veterinary vaccine adjuvants. Int J Parasitol, 2003. 33(5-6): p. 469-78.

53. Hong, G., et al., Effect of PMMA polymer on the dynamic viscoelasticity and plasticizer leachability of PEMA-based tissue conditioners. Dent Mater J, 2010. 29(4): p. 374-80.

54. Royals, M.A., et al., Biocompatibility of a biodegradable in situ forming implant system in rhesus monkeys. J Biomed Mater Res, 1999. 45(3): p. 231-9.

55. ICH Q3C Guideline, Impurities: Residual solvents., in http://www.emea.europa.eu/pdfs/human/ich/028395en.pdf., FDA, Editor. 
56. Grodowska, K. and A. Parczewski, Organic solvents in the pharmaceutical industry. Acta Pol Pharm, 2010. 67(1): p. 3-12.

57. Das, P., Revolutionary vaccine technology breaks the cold chain. Lancet Infect Dis, 2004. 4(12): p. 719. 


\section{VITA}

Qiuye Zhang was born in Nenjiang, China in 1973. After graduating from high school in 1992, he attended the 7-year program of clinical medicine in Harbin Medical University, where he received his MD degree in 1999. After graduation, he completed three years of residency training in the Second Hospital of Harbin Medical University in 2002 and practiced in OB/Gyn in the Second Hospital of Harbin Medical University for one year. In August 2007, he began his doctoral education in the Pharmaceutical Sciences Program in the University of Tennessee, Health Science Center, Memphis, from where he will obtain his PhD in Pharmaceutical Sciences in May 2014. 